\title{
The Extremely Luminous Quasar Survey in the Pan-STARRS 1 Footprint (PS-ELQS)
}

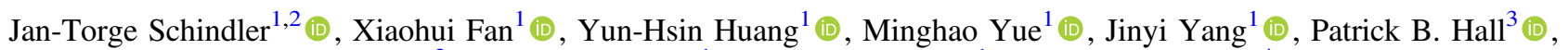 \\ Lukas Wenzl $^{2}$ (D), Allison Hughes ${ }^{1}$ (D), Katrina C. Litke ${ }^{1}$ (D), and Jon M. Rees ${ }^{4}$ (D) \\ ${ }^{1}$ Steward Observatory, University of Arizona, 933 North Cherry Avenue, Tucson, AZ 85721, USA \\ ${ }^{2}$ Max Planck Institute for Astronomy, Königstuhl 17, D-69117 Heidelberg, Germany \\ ${ }^{3}$ Department of Physics and Astronomy, York University, Toronto, ON M3J 1P3, Canada \\ ${ }^{4}$ Center for Astrophysics and Space Science, University of California San Diego, La Jolla, CA 92093, USA \\ Received 2019 March 2; revised 2019 April 27; accepted 2019 May 2; published 2019 July 1
}

\begin{abstract}
We present the results of the Extremely Luminous Quasar Survey in the $3 \pi$ survey of the Panoramic Survey Telescope and Rapid Response System (Pan-STARRS; PS1). This effort applies the successful quasar selection strategy of the Extremely Luminous Survey in the Sloan Digital Sky Survey footprint $\left(\sim 12,000 \mathrm{deg}^{2}\right)$ to a much larger area $\left(\sim 21,486 \mathrm{deg}^{2}\right)$. This spectroscopic survey targets the most luminous quasars $\left(M_{1450} \leqslant-26.5\right.$; $\left.m_{i} \leqslant 18.5\right)$ at intermediate redshifts $(z \geqslant 2.8)$. Candidates are selected based on a near-infrared $J K W 2$ color cut using WISE AllWISE and 2MASS photometry to mainly reject stellar contaminants. Photometric redshifts $\left(z_{\text {reg }}\right)$ and star-quasar classifications for each candidate are calculated from near-infrared and optical photometry using the supervised machine learning technique random forests. We select 806 quasar candidates at $z_{\text {reg }} \geqslant 2.8$ from a parent sample of 74,318 sources. After exclusion of known sources and rejection of candidates with unreliable photometry, we have taken optical identification spectra for 290 of our 334 good PS-ELQS candidates. We report the discovery of 190 new $z \geqslant 2.8$ quasars and an additional 28 quasars at lower redshifts. A total of 44 good PSELQS candidates remain unobserved. Including all known quasars at $z \geqslant 2.8$, our quasar selection method has a selection efficiency of at least $77 \%$. At lower declinations, $-30 \leqslant \mathrm{decl}$. $\leqslant 0$, we approximately treble the known population of extremely luminous quasars. We provide the PS-ELQS quasar catalog with a total of 592 luminous quasars $\left(m_{i} \leqslant 18.5, z \geqslant 2.8\right)$. This unique sample will not only be able to provide constraints on the volume density and quasar clustering of extremely luminous quasars, but also offers valuable targets for studies of the intergalactic medium.
\end{abstract}

Key words: galaxies: nuclei - quasars: general

Supporting material: machine-readable tables

\section{Introduction}

Quasars are excellent tracers of the formation and evolution of highly accreting supermassive black holes (SMBHs) across cosmic time. Their large luminosities not only allow us to detect and study them within the first billion years of the universe (e.g., Fan et al. 2000), but further provide strong background sources with which one can probe the large-scale structure formation of the universe and the nature of the intergalactic medium (IGM) (e.g., Simcoe et al. 2004; Prochaska et al. 2005). The highest-redshift quasars at $z \geqslant 7$ provide strong constraints on the reionization of the universe (Mortlock et al. 2011; Bañados et al. 2018; Wang et al. 2018b; Matsuoka et al. 2019) and on models of SMBH formation (Volonteri 2012).

Large quasar surveys provide the necessary number statistics to study the evolution of active SMBHs. The Sloan Digital Sky Survey (SDSS; York et al. 2000), the Baryon Oscillation Spectroscopic Survey (BOSS; Eisenstein et al. 2011; Dawson et al. 2013) and the extended BOSS (eBOSS; Dawson et al. 2016) have identified over 500,000 quasars at $z \lesssim 6$ and dozens of quasars at $z>6$ (Fan et al. 2001, 2003, 2004, 2006; Jiang et al. 2008, 2009, 2016).

Efforts at higher redshifts have also utilized other large surveys like the CFHQS (e.g., Willott et al. 2007, 2010), UKIDSS (e.g., Venemans et al. 2007; Mortlock et al. 2011), VIKING (Venemans et al. 2013), VST-ATLAS (Carnall et al. 2015; Chehade et al. 2018), DES (Reed et al. 2015, 2017, 2019;
Yang et al. 2019a), Pan-STARRS1 (Morganson et al. 2012; Bañados et al. 2014, 2016; Mazzucchelli et al. 2017; Pons et al. 2019) and the DESI Legacy Imaging Surveys (Wang et al. 2018a). The Hyper Surprime-Cam Subaru Strategic program (Aihara et al. 2018) allowed the exploration of the fainter quasar population at intermediate (Akiyama et al. 2018) and high redshifts (Kashikawa et al. 2015; Matsuoka et al. 2016, 2018a, 2018b).

While recent efforts have mainly focused on the highredshift quasar regime, surveys to identify intermediate-redshift quasars outside the SDSS footprint have been scarce. In particular, the $3 \pi$ wide area coverage of the Pan-STARRS1 (Panoramic Survey Telescope and Rapid Response System, Kaiser et al. 2002, 2010) survey (PS1; Chambers et al. 2016) provides an excellent opportunity to explore the extremely luminous quasar population $\left(M_{1450} \lesssim-28\right)$.

While these extremely luminous quasars at intermediate redshift are similarly rare as high-redshift quasars $\left(10^{-9} \mathrm{Mpc}^{-3} \mathrm{mag}^{-1}\right.$; see, e.g., Ross et al. 2013), they are valuable sources to study the He reionization of the universe (Worseck \& Prochaska 2011; Worseck et al. 2016), to explore the ionization state of the IGM (Schmidt et al. 2018), to investigate quasar clustering (e.g., Myers et al. 2006), and to constrain the evolution of the bright end of the quasar population (Schindler et al. 2018).

In this work we build on the Extremely Luminous Quasar Survey in the SDSS footprint (ELQS; Schindler et al. 2017, hereafter ELQS1) to discover $z=2.8-5$ quasars with $m_{i} \leqslant$ 18.5 in $\sim 21,486 \mathrm{deg}^{2}$ of the PS1 $3 \pi$ footprint. 
We first describe the photometry that the quasar selection is based on (Section 2) and give an overview of quasar catalogs in the literature that we use (Section 3). We subsequently present our quasar selection strategy in Section 4 and the construction of the PS-ELQS candidate catalog in Section 5. Section 6 discusses the spectroscopic observations and the data reduction, before we present the PS-ELQS quasar catalog in Section 7. We discuss our results in Section 8 and provide a summary in Section 9. Discovery spectra and tables detailing properties of the newly discovered quasars are available in the Appendices.

We present magnitudes in the $\mathrm{AB}$ system (Oke \& Gunn 1983), which are corrected for Galactic extinction (Schlegel et al. 1998). All optical passbands refer to PS1, unless otherwise noted. Extinction-corrected magnitudes are denoted by $m_{x}$, where $x$ refers to the photometric band, as opposed to extinction-uncorrected magnitudes $x$. We employ a standard $\Lambda \mathrm{CDM}$ cosmology with $H_{0}=70 \mathrm{~km} \mathrm{~s}^{-1} \mathrm{Mpc}^{-1}, \Omega_{m}=0.3$ and $\Omega_{\Lambda}=0.7$, generally consistent with recent measurements (Planck Collaboration et al. 2016).

\section{Photometry}

\subsection{The Wide-field Infrared Survey Explorer}

Our quasar selection takes advantage of the Wide-field Infrared Survey Explorer (WISE) AllWISE data release, providing infrared photometry over the entire sky at 3.4, 4.6, 12 , and $22 \mu \mathrm{m}(W 1, W 2, W 3, W 4)$. AllWISE combines data from the original cryogenic mission and its post-cryogenic extension (Mainzer et al. 2011). ${ }^{5}$ For our selection process we use the $W 1(3.4 \mu \mathrm{m})$ and $W 2(4.6 \mu \mathrm{m})$ photometry, for which the AllWISE source catalog achieved $95 \%$ photometric completeness for all sources with limiting magnitudes brighter than 19.8, 19.0 (Vega: 17.1, 15.7), respectively. Vega magnitudes were converted to the $\mathrm{AB}$ magnitude system using $W 1_{\mathrm{AB}}=W 1_{\text {Vega }}+2.699$ and $W 2_{\mathrm{AB}}=W 2_{\mathrm{Vega}}+3.339$ and extinction-corrected using $A_{W 1}, A_{W 2}=0.189,0.146$.

\subsection{The Two Micron All Sky Survey}

We extend the WISE photometry to the near-infrared taking advantage of the Two Micron All Sky Survey (2MASS), which mapped the entire sky in the near-infrared bands $J(1.25 \mu \mathrm{m}), H$ $(1.65 \mu \mathrm{m})$, and $K_{s}(2.17 \mu \mathrm{m})$. The 2MASS point-source catalog (PSC) includes all sources detected with a singal-to-noise ratio $\mathrm{S} / \mathrm{N} \geqslant 7$ in one band or $\mathrm{S} / \mathrm{N} \geqslant 5$ detections in all three bands. Unfortunately, due to strong confusion of sources closer to the Galactic plane, the photometric sensitivity is a strong function of Galactic latitude. Generally, all sources brighter than 16.7, 16.4, 16.1 (Vega: $15.8,15.0,14.3$ ) in the $J, H$ and $K_{s}$ bands are detected with $10 \sigma$ photometric sensitivity. However, based on the on-line documentation ${ }^{6}$ we estimate the $10 \sigma$ limiting magnitudes for higher latitudes to be $J=17.7, H=17.5$, $K_{s}=17.1$. Conveniently, the 2MASS PSC has been prematched to the WISE AllWISE source catalog. The match corresponds to the closest 2MASS object within a $3^{\prime \prime}$ radius of the WISE position. All 2MASS Vega magnitudes are converted to the $\mathrm{AB}$ system using $J_{\mathrm{AB}}=J_{\mathrm{Vega}}+0.894, H_{\mathrm{AB}}=H_{\mathrm{Vega}}+$

\footnotetext{
5 http://irsa.ipac.caltech.edu/cgi-bin/Gator/nph-scan?submit=Select\& projshort $=$ WISE

Figure 7 on https://www.ipac.caltech.edu/2mass/releases/allsky/doc/sec2 2.html.
}

$1.374, K_{s, \mathrm{AB}}=K_{s, \text { Vega }}+1.84$ and corrected for Galactic extinction $\left(A_{J}, A_{H}, A_{K_{s}}=0.723,0.460,0.310\right)$.

\subsection{The PS1}

We combine the near-infrared/infrared photometry of 2MASS and WISE by DR1 optical photometry from the PS1 $3 \pi$ survey (Chambers et al. 2016). PS1 delivers optical photometry in the $g_{-}, r-, i-, z_{-}^{-}$, and $y$-bands up to a depth of $23.3,23.2,23.1,22.3$, and 21.3 magnitudes $(5 \sigma, 3 \pi$ stack $)$ over $3 \pi$ sr of the sky (decl. $>-30$ ). Saturation only occurs at magnitudes of $\sim 12-14$, depending on the seeing conditions. The PS1 photometry is nominally on the $\mathrm{AB}$ system. All magnitudes are corrected for Galactic extinction $\left(A_{g}, A_{r}, A_{i}, A_{z}\right.$, $\left.A_{y}=3.172,2.271,1.682,1.322,1.087\right)$.

\section{Quasar Catalogs in the Literature}

To match promising candidates with known quasars from the literature, we make use of the large quasar samples discovered by SDSS I/II (Abazajian et al. 2009), BOSS, and eBOSS published in the SDSS DR7 (DR7Q; Schneider et al. 2010), DR12 (DR12Q; Pâris et al. 2012) and DR14 (DR14Q; Pâris et al. 2018) quasar catalogs.

The quasar selection for the SDSS I/II spectroscopic survey is described in Richards et al. (2002) and selects quasars as outliers of the stellar locus in the ugri and griz color space. Inclusion regions are designed to include quasars in certain redshift ranges which are highly contaminated with stellar sources. The resulting DR7Q includes 100,000 quasars over $9380 \mathrm{deg}^{2}$ region of the SDSS DR7 footprint. The BOSS quasar selection (Bovy et al. 2011) was optimized to find quasars in the targeted redshift range of BOSS at $2.2<$ $z<3.5$. The newly discovered quasars in BOSS were published in DR12Q. The eBOSS quasar selection (Myers et al. 2015) is based on the XDQSO method (Bovy et al. 2011) and a mid-infrared color cut to provide a uniform quasar sample over $7500 \mathrm{deg}^{2}$ with $g_{\text {SDSS }}<22$ or $r_{\text {SDSS }}<22$.

The latest version of the SDSS quasar catalog (DR14Q) was then designed to include all quasars observed during any of the stages of the SDSS. Therefore the DR14Q includes nearly all of the DR7Q and DR12Q quasars. All in all, the SDSS discovered more than 500,000 quasars in the northern hemisphere and makes up the majority of the known quasars in the PS1 footprint. We use all three SDSS quasar catalogs mentioned above to match our candidate sample against known sources. Furthermore, the quasar training set for the random forest regression and classification is built from DR7Q and DR12Q quasars.

In addition to the SDSS quasar catalogs we also match our candidates against the Million Quasar Catalog (MQC, version 5.7b; Flesch 2015). The MQC is a compilation of type I and type II active galactic nuclei from all the available literature, including a large fraction of quasar candidates. All quasars from the SDSS quasar catalogs can also be found within the MQC. For the cross-match to our candidate list we exclude all quasar candidates.

J. Yang et al. (2019, in preparation) are also working on a spectroscopic survey of bright quasars at intermediate redshifts similar to PS-ELQS. They are exploring two quasar selections (Wu \& Jia 2010; Wu et al. 2012) targeted at $z \approx 2-3$ and at $z \geqslant 4$ to assess different selection criteria for the upcoming LAMOST quasar survey. The spectroscopic identification 


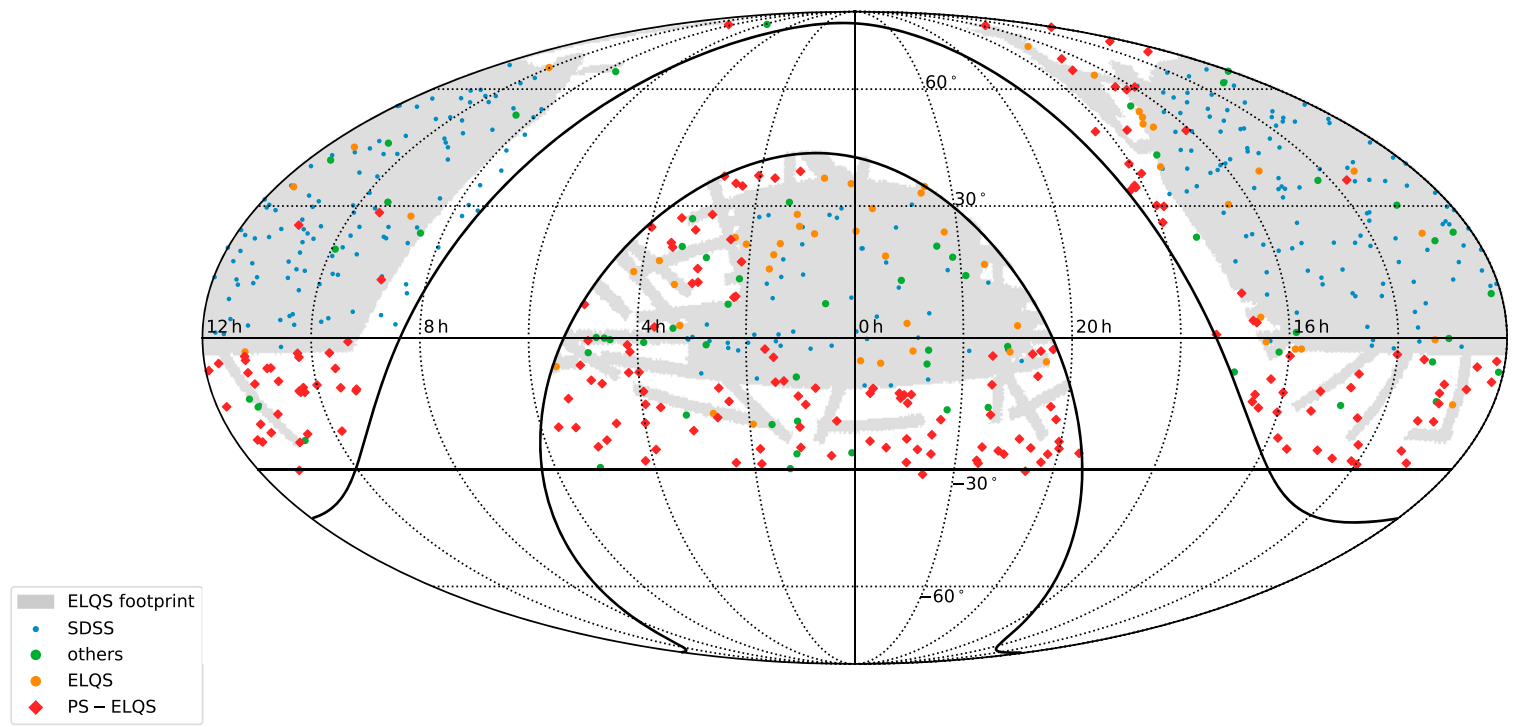

Figure 1. Mollweide projection of the entire sky in equatorial coordinates. The original ELQS footprint is shown in gray. The PS-ELQS footprint covers all area above the thick solid line at decl. $=-30$ and outside of the two lines outlining the Galactic plane $(b \mid \leqslant 20)$. Data points show the position of all 592 quasars in the PSELQS quasar catalog (Section 7) colored by their reference.

campaigns were carried out with the Lijiang telescope $(2.4 \mathrm{~m})$ and the Xinglong telescope $(2.16 \mathrm{~m})$. A number of PS-ELQS candidates were spectroscopically identified by their efforts.

\section{The PS-ELQS Quasar Selection}

The PS-ELQS quasar selection is in many aspects analogous to the original ELQS quasar selection (see Sections 4-6, ELQS1). However, the optical photometry from PS1 does not provide $u$-band measurements like the SDSS did. While our initial near-infrared $J K W 2$ color-cut selection is not affected by this, we lose information on quasars, where the broad emission lines transition from the $u$-band to the $g$-band. As a consequence our photometric redshift estimates for quasars at $z \lesssim 3$ might be more unreliable. The additional $y$-band filter of PS1, which is essential for high-redshift quasar selections, only adds little information for the search of intermediate-redshift quasars.

The following subsections describe the PS-ELQS footprint as well as the individual steps of our quasar selection strategy in detail.

\subsection{PS-ELQS Footprint}

The ELQS aimed to discover extremely bright, intermediateredshift quasars, which are very rare. The survey used optical photometry provided by the SDSS over $\sim 12.000 \mathrm{deg}^{2}$, excluding the Galactic plane. With the public data release of the PS1 $3 \pi$ survey we can extend our previous efforts to a much larger area and into regions which have not been included in previous quasar surveys. Therefore PS-ELQS is designed to cover the entire PS1 footprint except the Galactic plane $(|b| \leqslant 20)$. Compared to the ELQS footprint we cover an additional $\sim 9600 \mathrm{deg}^{2}$, of which the majority $\left(\sim 5600 \mathrm{deg}^{2}\right)$ lies at $-30 \leqslant$ decl. $\leqslant 0$.

Figure 1 shows a Mollweide projection of the entire sky in the equatorial coordinate system. The coverage of the original ELQS is shown in gray. Two thick solid lines map the outline of the Galactic plane $(|b| \leqslant 20$ ), which we exclude from our selection. A third solid line at decl. $=-30$, shows the southern border of the PS1 footprint. We effectively select the all the area above decl. $=-30$ and outside of $|b| \leqslant 20$ for the PSELQS survey. Colored data points show all 592 quasars from the PS-ELQS quasar catalog described in Section 7. The color refers to their source of identification.

We employ the Hierarchical Equal Area isoLatitude Pixelization (HEALPix; Górski et al. 2005) to roughly estimate the area of the PS-ELQS footprint in a similar way to Jiang et al. (2016). HEALPix divides the sky into a grid of curvilinear, equal-sized quadrilaterals. At the lowest resolution the sky is represented by 12 pixels. To create higher-resolution maps each pixel is subdivided into four pixels per resolution level. Therefore the total number of pixels follows $N_{\text {pix }}=12 \cdot 2^{\text {lv1 }}$.

Our coverage estimate is based on 1.918 .290 sources selected by our photometric $J K W 2$ color-cut selection (Section 4.2), which were matched to PS1 according to our quality criteria but without enforcing the extended object rejection. We expect the PS-ELQS footprint to roughly cover $\sim 20,000 \mathrm{deg}^{2}$, resulting on average in one source per $\sim 0.01 \mathrm{deg}^{2}$. We choose resolutions with $\mathrm{lvl}=6,7,8,9$ to calculate our coverage maps, resulting in a total number of pixels of 49,152, 196,608, 786,432, and 3145,728 with $\sim 0.84$, $0.02,0.05$, and $0.01 \mathrm{deg}^{2}$ per pixel. Our coverage estimates for these three resolution levels are 21,697, 21,487, 20,676, and $13,501 \mathrm{deg}^{2}$, respectively. There is a large decrease in coverage from lvl $=8$ to 9 . At the highest resolution the pixel density is approaching the source density and we are effectively oversampling the area.

We adopt a resolution of $l v l=7$ with an effective area of $21,486_{-833}^{+210} \mathrm{deg}^{2}$ for our final coverage estimate. The uncertainties reflect the differences to the coarser $(l v l=6)$ and finer $(\mathrm{lvl}=8)$ resolutions.

\subsection{Photometric Selection}

We begin our photometric selection with the WISE AllWISE catalog pre-matched to all sources from the 2MASS PSC. An overview of the selection process is given in Section 5 . The source selection is restricted to higher Galactic latitudes 
$(|b| \geqslant 20)$ to exclude the Galactic plane, where a high source density leads to significant source confusion. The selection further requires $\mathrm{S} / \mathrm{N} \geqslant 5$ in the WISE $W 1$ and $W 2$ bands and $J$-band detections $(J>0)$ for all objects. At the heart of the near-infrared selection is the $J K W 2$ color cut (ELQS1),

$$
K_{\text {Vega }}-W 2_{\text {Vega }} \geqslant 1.8-0.848 \cdot\left(J_{\text {Vega }}-K_{\text {Vega }}\right) \text {, }
$$

which allows us to clearly separate quasars at $z<5$ from the stellar locus in $J-K-W 2$ color-space.

We obtained 3815,192 sources, which were then further matched to optical photometry from the Pan-STARRS PS1 catalog within a 3".96 aperture using the STSCI MAST casjobs interface. ${ }^{7}$ We adopt the flags outlined in Bañados et al. (2014, their Table 6) to ensure the selection of reliable photometry according to the Image Processing Pipeline (Magnier 2006, 2007). The full SQL query to retrieve the PS1 data is provided in Appendix E. We have included a loose criterion to reject extended sources (Section 4.3) in the query to reduce the download size of the data set. The match to the Pan-STARRS PS1 catalog returned a total of 74,318 sources.

The Pan-STARRS PS1 photometry was matched with the AllWISE position. While the AllWISE point-spread function (PSF) is larger than the 2MASS PSF, their average astrometric precision with respect to the U.S. Naval Observatory CCD Astrograph Catalog is similar (2MASS: $\sim 80$ mas, AllWISE: $\sim 87$ mas). However, the 2MASS PSC online documentation ${ }^{8}$ notes that stars fainter than $K_{\text {Vega }} \sim 14$ have worse position residuals, which indicates that the extraction uncertainties dominate rather than the uncertainties in the mapping into the IRCS reference frame. As our selection is limited by the depth of the 2MASS survey, and the majority of the pre-matched 3815,192 sources have $K_{\text {Vega }}>14$, we preferred to use the AllWISE position over the 2MASS position for the crossmatch to PanSTARRS PS1.

The optical and near-infrared photometry is extinctioncorrected using the python dustmaps module (Green et al. 2018) with the values of Schlegel et al. (1998).

\subsection{Rejection of Extended Sources}

The $J K W 2$ color cut is highly successful in rejecting stellar contaminants. However, as described in ELQS1, galaxies straddle the color cut and become our main contaminants once the majority of stars are excluded.

We use the absolute value of the magnitude difference $(\Delta m)$ between the PS1 mean PSF magnitudes (iMeanPSFMag) and the PS1 mean aperture magnitudes (iMeanApMag) as our main quantity to identify extended sources.

In Figure 2(a) we display two data sets as a function of their magnitude difference, $\Delta m$. The first histogram (blue solid line) is calculated from all sources in a region of $b \leqslant-20$ $120 \leqslant l \leqslant 240$ (Galactic coordinates) that passed the JKW2 color cut and were matched to PS1 photometry according to our criteria above. This corresponds to roughly $2250 \mathrm{deg}^{2}$ or $\sim 10 \%$ of the total survey area. There are 267,951 sources in this data set, of which $12,579(15,696)$ have $\Delta m \leqslant 0.15(0.3)$. The distribution of the sources as a function of $\Delta m$ has a minimum around $\Delta m \approx 0.2$, with the majority of sources in the data set exhibiting higher values of $\Delta m$. The second data set contains the quasars from the combined SDSS DR7 and

\footnotetext{
http://mastweb.stsci.edu/ps1casjobs/

8 https://old.ipac.caltech.edu/2mass/releases/allsky/doc/sec2_2.html
}
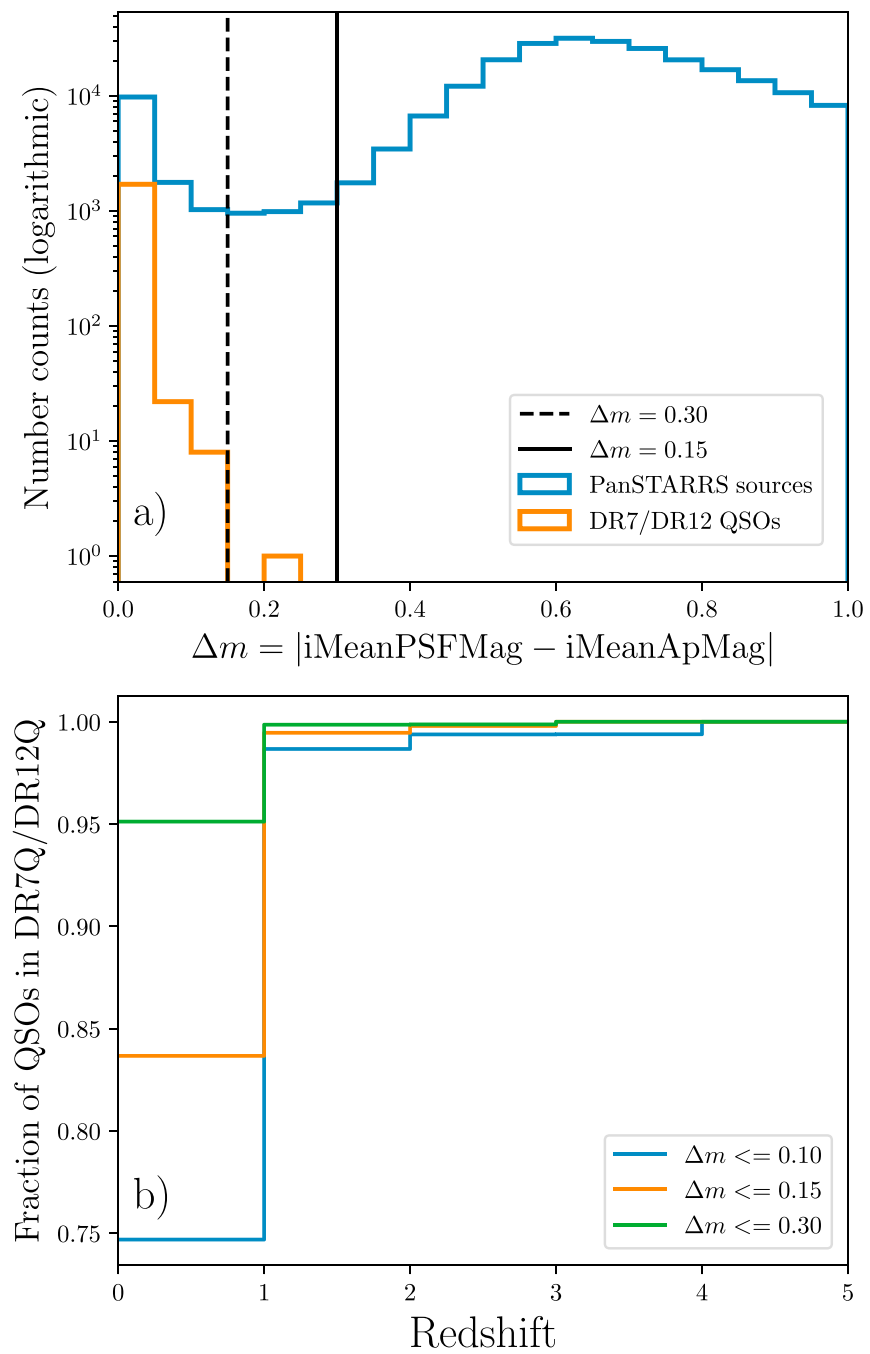

Figure 2. (a) Distribution of PS1 sources (blue) and SDSS DR7Q/DR12Q quasars (orange) as a function of the absolute value of the difference $(\Delta m)$ between the PS1 mean PSF magnitude and the PS mean aperture magnitude. The PS1 sources are selected by the photometric criteria discussed in Section 4.2 in a region of $b \leqslant-20$ and $120 \leqslant l \leqslant 240$ (Galactic coordinates). The SDSS quasars are restricted to $i \leqslant 18.5$ and to redshifts $z \geqslant 2.5$, as targeted in this study. (b) The fraction of bright $(i \leqslant 18.5)$ quasars in the DR7Q/DR12Q included in three different $\Delta m$ cuts as a function of redshift. At redshifts $z \geqslant 1.0$ the majority of all quasars are unaffected by the $\Delta m$ cut.

SDSS DR12 quasar catalogs matched to PS1 photometry. In addition, we only include quasars with $i<18.5$ and (visually inspected) $z>2.5$ to restrict the quasar sample to the same magnitude range and redshift range that we target with our selection. All of the remaining 1736 quasars have $\Delta m<0.3$ and all except one are even included within $\Delta m<0.15$.

In Figure 2(b) we display the fraction of quasars that are restricted by various cuts on $\Delta m$ to all SDSS DR7/DR12 quasars with $m_{\mathrm{i}}<18.5$. It becomes evident that any of the three restrictions on $\Delta m$ decreases the number of quasars at the lowest redshifts. At redshifts beyond $z \approx 1.0$ the majority of quasars $(>99 \%)$ are included even when the stronger restriction of $\Delta m \leqslant 0.15$ is applied.

For the PS-ELQS quasar selection we thus reject extended sources using $\Delta m<0.15$ as our main criterion. Based on the SDSS DR7/DR12 quasar samples we estimate that this restriction has a completeness of $99.8 \%$ for quasars at 
Table 1

Overview over the Number of Quasars and Stars in the Training Sets

\begin{tabular}{|c|c|c|}
\hline Data Set & DR13 Stars & DR7/DR12 Quasars \\
\hline Full catalogs & 383,966 & 213,781 \\
\hline \multicolumn{3}{|c|}{ Classification } \\
\hline $\mathrm{PS} 1, i_{\mathrm{SDSS}}<21.5$ & 335,591 & 153,020 \\
\hline PS $1+W 1 W 2, i_{\text {SDSS }}<21.5$ & 233,137 & 133,600 \\
\hline PS1+TMASS $+W 1 W 2, i_{\text {SDSS }}<21.5$ & 169,335 & 5926 \\
\hline \multicolumn{3}{|c|}{ Regression } \\
\hline $\mathrm{PS} 1+W 1 W 2$ & ... & 134,097 \\
\hline $\mathrm{PS} 1+W 1 W 2, i_{\mathrm{SDSS}}<18.5$ & $\cdots$ & 13,119 \\
\hline
\end{tabular}

$z>2.5$. After applying the $m_{\mathrm{i}}<18.5$ magnitude cut and the criterion to reject extended objects to our 74,318 candidates, we retain 43,430 sources.

\subsection{Random Forest Redshift Regression and Classification}

Random forests (Breiman 2001) are a supervised machine learning technique, that can be efficiently applied to multi-class classification or standard regression problems. The algorithm is non-parametric and avoids the problem of overfitting. In the past random forests have been successfully used on many astronomical data sets (Carliles et al. 2010; Dubath et al. 2011; Richards et al. 2011; Carrasco Kind \& Brunner 2013), including quasar classification (Carrasco et al. 2015) and redshift estimation (D'Isanto \& Polsterer 2018).

For any supervised machine learning technique, the results of the classifier (or regressor) are highly dependent on the training set. For our purposes we base our training set on quasars from the combined SDSS DR7 and DR12 quasar catalogs as well as on a spectroscopic sample of stars from SDSS DR13. These are essentially the same training sets used in ELQS1, matched to the PS1 source catalog within 3"!96 to obtain PS1 DR1 photometry. Table 1 provides the total numbers of stars and quasars in the different training sets used below.

After the photometric selection and the extended object rejection our candidate sample is still contaminated by stars and low-redshift $(z<2.8)$ quasars. To enhance our efficiency we use the random forest classifier to reject obvious stellar contaminants and then estimate a photometric redshift with the random forest regressor to select only the $z \geqslant 2.8$ quasars.

We use the scikit-learn (Pedregosa et al. 2011) python implementation of the random forest classifier and regressor with its default parameters unless otherwise noted.

\subsubsection{Classification}

To further enhance the efficiency of our selection we employ random forest classification. We classify our candidates into four different redshift classes ("vlowz": $0<z \leqslant 1.5$, "lowz": $1.5<z \leqslant 2.2$, "midz": $2.2<z \leqslant 3.5$, "highz": $3.5<z)$ and five different stellar spectral classes (A, F, G, K, M). The redshift classes are designed to split the quasars at redshifts where emission lines move from one passband into the next redder passband, introducing strong features in the corresponding flux ratios (see also Richards et al. 2015). For evaluation purposes we also summarize all stellar classes under the "STAR" label and all quasar classes under the "QSO" label, effectively resulting in a binary classification.

We combine the SDSS DR7Q/DR12Q quasars with the SDSS DR13 spectroscopic stars (see ELQS1) matched to PS1 photometry to form the classification training set. Our only requirement is that all objects be brighter than $i_{\text {SDSS }}<21.5$ to exclude very faint objects with substantial photometric uncertainties.

We test the performance of the classification for three subsets of the full training set with different features. The first uses the four adjacent flux ratios $(g / r, r / i, i / z, z / y)$ of the five photometric PS1 bands and the PS1 $i$-band magnitude as features. For the second and third training set we first include the WISE $W 1$ and $W 2$ bands (PS1+W1W2) and for the third we also include all three 2MASS passbands (PS1+TMASS+ $W 1 W 2$ ). The feature set is expanded accordingly, when we include the WISE and 2MASS photometry, by adding the additional flux ratios $\left(+[y / W 1, W 1 / W 2] ;+\left[y / J, J / H, H / K_{s}\right.\right.$, $\left.\left.K_{s} / W 1, W 1 / W 2\right]\right)$ and the $W 1$ and the $J$-band magnitude. We require each object in the subsets to have information in all used features (see the constraints in Table 2), resulting in varying number of sources per data set. For each subset we calculate the best combination of hyperparameters for the classifier on a grid of $n_{-}$estimators $=[200,300,400]$, min_samples_splot $=[2,3,4]$, and max_depth $=[15$, $20,25]$. As in the case for the photometric redshift regression, we apply five-fold cross-validation on the full training set using $80 \%$ of the sources for training and the remaining $20 \%$ for validation.

The best hyperparameters are evaluated using the $F_{1}$ score (Bishop 2006). The $F_{1}$ score, which is also called the traditional $F$-measure or the balanced $F$-score, is the harmonic mean of the precision and the recall of the classification:

$$
F_{1}=2 \cdot \frac{\text { precision } \cdot \text { recall }}{\text { precision }+ \text { recall }} .
$$

Here precision ( $p$; or efficiency) is defined as the ratio of true positives to the sum of true and false positives and the recall $(r$; or completeness) is defined as the ratio of true positives to the sum of true positives and false negatives.

Table 2 provides an overview of the best classification results for the three different subsets. The first three columns show the sizes of the training and validation sets for each subset, the constraints on the subset, and the features used. The last three columns provide the precision $(p)$, recall $(r)$ and $F_{1}$ measure for the "highz" quasar class as well as the binary classification between quasars ("QSO") and stars ("STAR"). We would like to stress that only 12 "highz" quasars were included in the PS $1+W 1 W 2+$ TMASS validation set (third row in Table 2) to determine the $p, r$ and $F_{1}$ values for the "highz" class, introducing high stochastic uncertainties on those values.

The inclusion of flux ratios beyond the PS1 photometry leads to generally better classification results. On the downside, the sample sizes decrease with the addition of the WISE and 2MASS photometry, as we require all objects to have information in all features considered. When the training sets become too small, they will not be able to fully populate the available feature space and thus will lead to worse classifications. Additionally, small validation sets will introduce large errors on the classification metrics. As a result, the recall value and $F_{1}$ score of the "highz" class in the PS $1+W 1 W 2+$ TMASS 
Table 2

Results of the Random Forest Classification on the Full Empirical Training Set

\begin{tabular}{|c|c|c|c|c|c|c|}
\hline $\begin{array}{l}\text { Training/Validation Size } \\
\end{array}$ & Constraints & Features $^{\mathrm{b}}$ & $p / r / F 1$ (highz) & $p / r / F 1(\mathrm{QSO})$ & $p / r / F 1$ (STAR) & \\
\hline $390888 / 97723$ & PS1 fl.r., $i_{\text {SDSS }}<21.5$ & PS1 & $0.87 / 0.85 / 0.86$ & $0.93 / 0.91 / 0.92$ & $0.96 / 0.97 / 0.97$ & \\
\hline 293389/73348 & PS1+W1 $W 2$ fl.r., $i_{\text {SDSS }}<21.5$ & $\mathrm{PS} 1+W 1 W 2$ & $0.93 / 0.93 / 0.93$ & $0.99 / 1.00 / 0.99$ & $1.00 / 0.99 / 0.99$ & \\
\hline $140208 / 35053$ & PS1+TMASS $+W 1 W 2$ fl.r., $i_{\text {SDSS }}<21.5$ & $\mathrm{PS} 1+\mathrm{TMASS}+W 1 W 2$ & $1.00 / 0.67 / 0.80(12)$ & $1.00 / 0.98 / 0.99$ & $1.00 / 1.00 / 1.00$ & \\
\hline
\end{tabular}

a Notes.

${ }^{a}$ For the five-fold cross-validation the full data sets are split into a training (80\%) and validation (20\%) set. We provide the number of objects for each set in this column.

b We abbreviated flux ratios to "fl.r." in this column. 


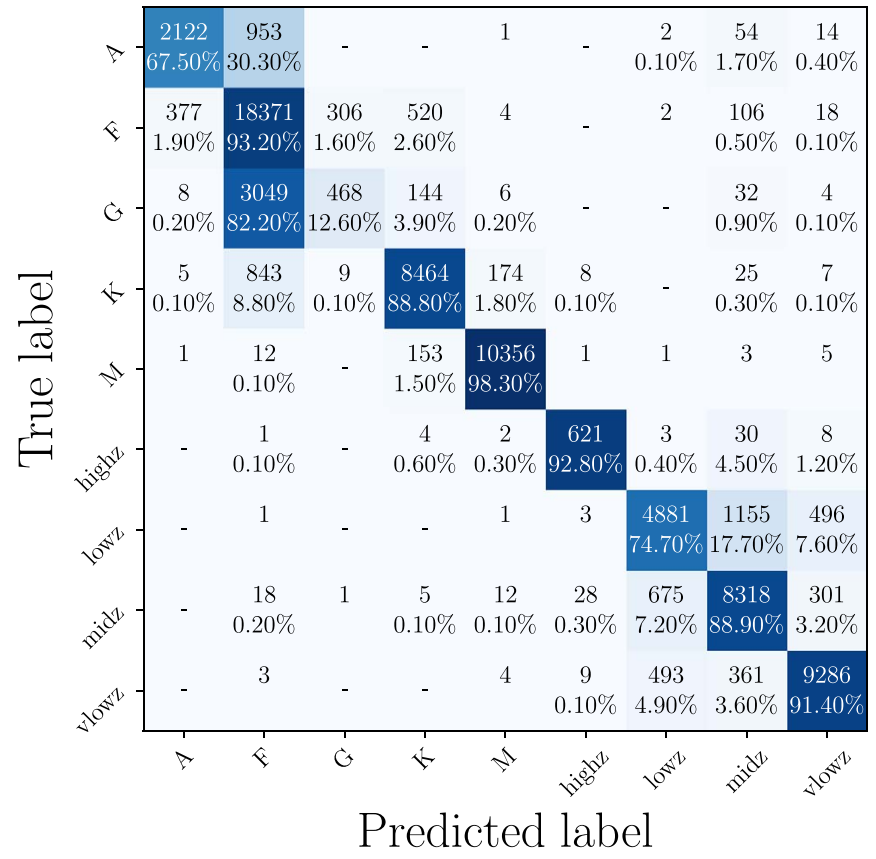

Figure 3. Confusion matrix for the PS1+W1W2 subset ( $\star$ in Table 2 ). The rows show the true labels (classes) of the objects, whereas the columns indicate the predicted labels (classes). Each entry shows the total number of objects of the true row label classified as the predicted column label. The percentages show the fraction of objects in that entry to the total number of objects in the row. The entries are color coded to highlight the entries with the majority of objects in each row.

set is worse than in the other two subsets, although more features are included.

Hence, we adopt the PS1+W1W2 subset as the best training and feature set for our classification problem (marked with $\star$ in Table 2). It achieves the best classification results of all three subsets. The best hyperparameters for this set were evaluated to be n_estimators $=400$, min_samples_splot $=4$, and max_depth $=25$.

A helpful visualization to understand the classification result is the confusion matrix. Each row of the matrix marks the true class of the objects within it, which are predicted to belong to different classes according to the columns of the matrix. The diagonal entries show the number of correctly classified objects, while all off-diagonal entries show the numbers of incorrectly classified objects. It provides a good overview of which classes are commonly confused and which can be separated easily with the features supplied to the classifier.

We display the entire confusion matrix for the PS1+W1W2 subset $(\star)$ in Figure 3 . The individual entries show the number of objects belonging to the row class (true label) and classified as the column class (predicted label). The percentages below each entry are with respect to the total number of objects in the true class (full row). Therefore the percentages of the diagonal entries show the completeness with respect to its row/column label.

The quasar and star classes are well separated. The largest number of stellar contaminants enter in the "midz" quasar class $(2.2 \leqslant z \leqslant 3.5)$. This is one redshift range, where the quasar distribution overlaps strongly with the stellar locus in optical color space. Indeed, the majority of quasars misclassified as stars also stem from this redshift class. Within the quasar classes "midz" and "lowz" quasars show the highest level of confusion with each other.

\subsubsection{Photometric Redshift Regression}

Our selection process rejects stars with the $J K W 2$ color cut and galaxies due to their large difference in mean PSF and aperture magnitudes. Therefore, quasars at $z<2.8$ become the dominant contaminants. We use random forest regression to calculate photometric redshifts, $z_{\text {reg }}$, and then select quasar candidates with $z_{\text {reg }} \geqslant 2.8$.

The training set includes all SDSS DR7/DR12 quasars with full PS1 and WISE $W 1$ and $W 2$ photometry. We also build a smaller subset, limiting the full quasar training set to $i<18.5$.

The features used for the random forest regression are the six adjacent flux ratios $(g / r, r / i, i / z, z / y, y / W 1, W 1 / W 2)$ from the five photometric bands of PS1 in addition to $W 1$ and $W 2$. We further add the PS1 $i$-band magnitude and the $W 1$ magnitude to the feature set. As discussed in ELQS1, including 2MASS photometry dramatically reduces the number of training objects and therefore does not allow for sufficient training in the large feature space.

We perform grid searches on the full training set and the magnitude-limited subsample to determine the hyperparameters of the best regression model. The grid of hyperparameters includes the number of binary trees (n_estimators $=[200$, $300,500]$ ), the minimum number of samples to be split (min_samples_splot $=[2,3,4])$, and the maximum depth of the tree (max_depth $=[15,20,25]$ ). To test the hyperparameters we use five-fold cross-validation on the full training set using each time $80 \%$ of the sources for training and the remaining $20 \%$ for validation.

The best hyperparameters are evaluated using the standard $R^{2}$ regression score,

$$
R^{2}=1-\frac{\sum_{i}\left(z_{\mathrm{spec}, i}-z_{\mathrm{reg}, i}\right)^{2}}{\sum_{i}\left(z_{\mathrm{spec}, i}-\bar{z}\right)^{2}},
$$

where $z_{\mathrm{spec}, i}$ are the true redshifts, $\bar{z}$ is the mean of all $z_{\mathrm{spec}, i}$, and the predicted redshift values are denoted by $z_{\text {reg, }, i}$. The other common metric in the literature assesses the goodness of the redshift estimation with redshift-normalized residuals $\left(\delta z=\left|z_{\text {reg }}-z_{\text {spec }}\right| /\left(1+z_{\text {spec }}\right)\right)$. Most studies report the fraction of quasars in the validation set with residuals smaller than a given threshold $e$ :

$$
\delta_{e}=\frac{N\left(\left|z_{\mathrm{reg}, i}-z_{\mathrm{spec}, i}\right|<e \cdot\left(1+z_{\mathrm{spec}, i}\right)\right)}{N_{\mathrm{tot}}},
$$

where $N_{\text {tot }}$ denotes the total number of quasars in the validation set. Residual thresholds of $e=0.1,0.2$, and 0.3 are typically chosen in this context.

We show the best results of the regression grid search for the full quasar training set and the magnitude-limited subsample in Table 3. While the magnitude-limited subset (second row) achieves slightly better results, the training set is reduced to $10 \%$ of its full size. In addition, it will be additionally biased against higher-redshift quasars, because they are generally fainter. Therefore we adopted the full quasar training set (marked with $\star$ in Table 3 ). The best hyperparameters for this case are min_samples_split $=2$, n_estimators $=500$, and max_depth $=25$. The resulting distribution of spectroscopic redshifts to random forest regression redshifts in the validation set are shown in Figure 4. While the results at $z \gtrsim 3$ are mostly within the region of $\Delta z=$ $\left|z_{\text {spec }}-z_{\text {reg }}\right| \leqslant 0.3$, a larger distribution of outliers persists at lower redshifts. 
Table 3

Results of the Photometric Redshift Estimation Methods

\begin{tabular}{|c|c|c|c|c|c|c|c|c|}
\hline Data Set & Training/Validation Size ${ }^{\mathrm{a}}$ & Constraints & Features & $\delta_{0.3}$ & $\delta_{0.2}$ & $\delta_{0.1}$ & $\sigma^{\mathrm{b}}$ & $R^{2}$ \\
\hline DR7DR12Q & $107277 / 26820$ & PS $1+W 1 W 2$ fl.r. & $\mathrm{PS} 1+W 1 W 2$ & 0.95 & 0.91 & 0.79 & 0.352 & 0.817 \\
\hline DR7DR12Q & $10495 / 2624$ & PS $1+W 1 W 2$ fl.r., $i<18.5$ & $\mathrm{PS} 1+W 1 W 2$ & 0.98 & 0.95 & 0.88 & 0.265 & 0.883 \\
\hline
\end{tabular}

Notes.

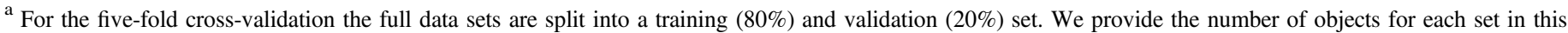
column.

${ }^{b}$ Standard deviation of the residual of the photometric redshift estimate (regression redshift) and the measured spectroscopic redshift.

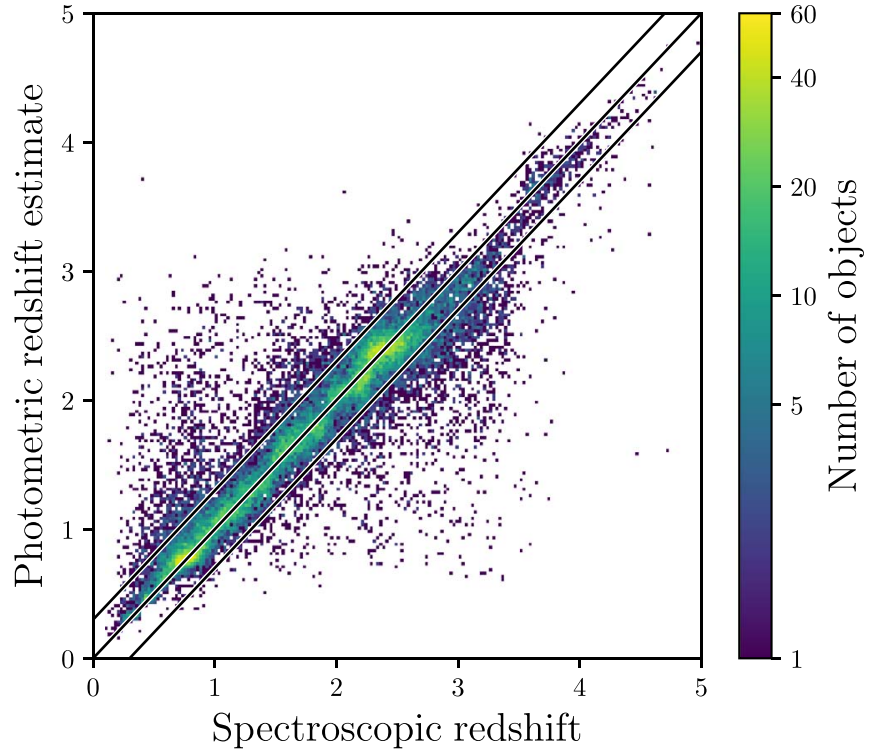

Figure 4. Distribution of quasars in the validation set ( $\star$ in Table 3 ) as a function of photometric redshift estimate (regression redshift) and measured spectroscopic redshift, color coded by the number of objects per bin. The three solid black lines illustrate the $\Delta z=\left|z_{\text {spec }}-z_{\text {reg }}\right|=0$ diagonal and the $\Delta z \leqslant 0.3$ region.

\section{Construction of the PS-ELQS Quasar Candidate Catalog}

We provide an overview of the candidate selection process in Figure 5 and Table 4 . The selection process begins with the parent sample of 2MASS and WISE AllWISE sources, which pass the $J K W 2$ color cut and have reliable photometry $(\mathrm{S} / \mathrm{N}$ $(W 1) \geqslant 5, \mathrm{~S} / \mathrm{N}(W 2) \geqslant 5, J>0)$. We then match these sources to the PS1 photometry within a 3".96 aperture, requiring the objects to be brighter than $i=19.0$ and fulfil $\Delta m \leqslant 0.3$. Furthermore all objects have to satisfy a range of quality flags. The full SQL query is shown in Appendix E. After we retrieved the PS1 photometry for all remaining sources $(74,318)$, we apply the more stringent rejection of extended sources $(\Delta m \leqslant 0.15)$ and restrict the sample to all sources with $i \leqslant 18.5$.

At this point we run the random forest regression and classification. The regression provides us with a regression redshift $z_{\text {reg }}$, our photometric redshift estimate. The classification determines the most likely class of the object ( $r f \_m u l t$ class_pred) and the summed probability of the object belonging to any of the quasar classes ( $\left.r f \_q s o \_p r o b\right)$. All objects that are generally classified as quasars ("QSO"), according to the binary classification, and have regression redshifts of $z_{\text {reg }} \geqslant 2.8$, form the PS-ELQS candidate sample.

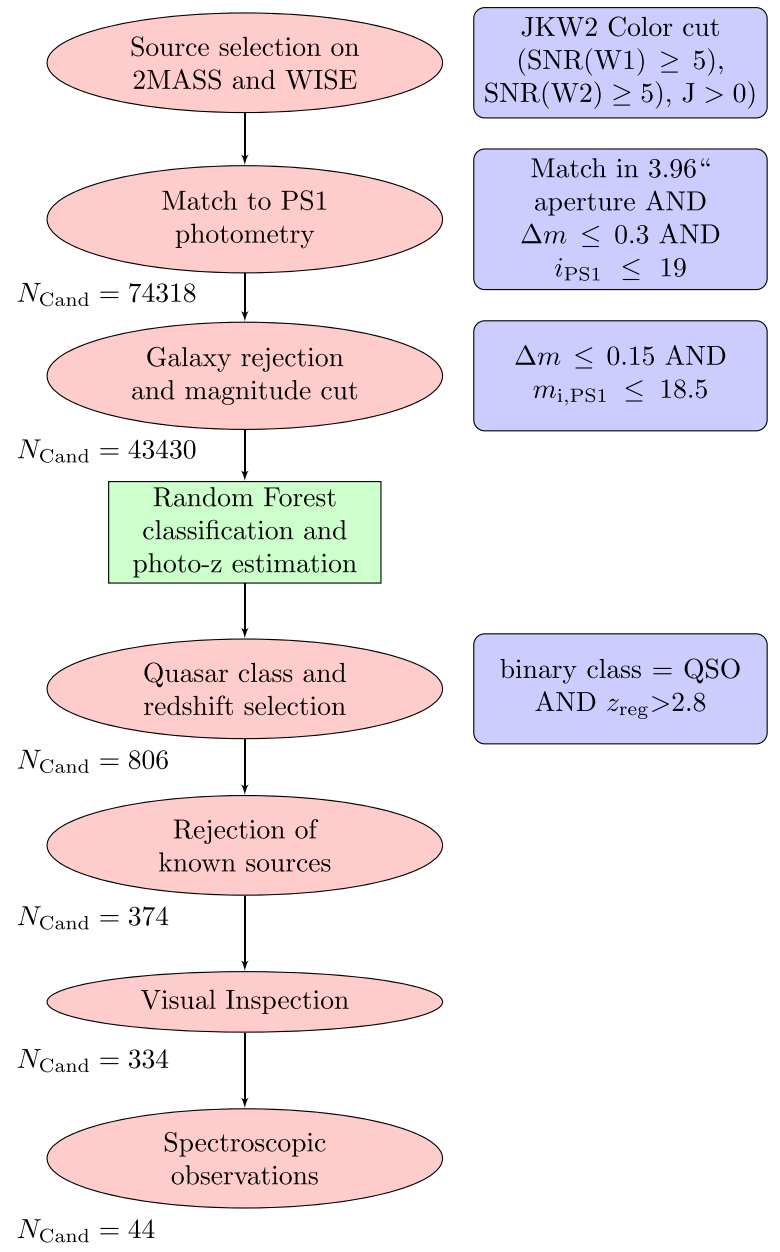

Figure 5. Flowchart of the PS-ELQS quasar candidate selection.

The PS-ELQS candidate sample selects a total of 432 known quasars from the literature, including 70 sources observed as part of the ELQS, which are then excluded from the candidate sample along with all other known sources. We visually inspect all unknown candidates for unreliable photometry. We reject all candidates whose PSF is blended with nearby sources, where image artifacts are evident or the source seems to be extended. After the exclusion of known quasars and the rejection of 40 candidates with unreliable photometry, the "good" PS-ELQS candidate sample has a total of 334 objects. These candidates are then prioritized according to the criteria described in Table 5. 
Table 4

PS-ELQS Candidate Selection

\begin{tabular}{lc}
\hline \hline $\begin{array}{l}\text { WISE+2MASS+PS1 parent sample } \\
(J K W 2 \text { color cut, } i<19.0,|\Delta m|<0.3)\end{array}$ & 74318 \\
\hline $\begin{array}{l}\text { Photometric sample } \\
(i<18.5,|\Delta m|<0.15)\end{array}$ & 43430 \\
\hline PS-ELQS candidate sample & 806 \\
$\left(\begin{array}{l}\text { reg } \geqslant 2.8 \text { and class }=\text { "QSO") } \\
\text { Known QSOs in the literature in the candidate sample }\end{array}\right.$ & 432 \\
Known $z \geqslant 2.8$ QSOs in the literature in the candidate sample & 402 \\
\hline Observed ELQS sources in the candidate sample & 70 \\
Known $z \geqslant 2.8$ ELQS QSOs in the candidate sample & 54 \\
\hline Good PS-ELQS candidates & 334 \\
(excluding bad photometry and known sources) & \\
\hline Good PS-ELQS candidates observed & 290 \\
Remaining good PS-ELQS candidates & 44 \\
\hline New PS-ELQS quasars & 218 \\
New PS-ELQS quasars at $z \geqslant 2.8$ & 190 \\
\hline
\end{tabular}

Table 5

PS-ELQS Quasar Candidates and Their Selection Priorities

\begin{tabular}{lcc}
\hline \hline Priority & Criteria & $\begin{array}{c}\text { Good PS-ELQS } \\
\text { Candidates } \\
(44 \text { remaining })\end{array}$ \\
\hline 1 & $3.5 \leqslant z_{\text {reg }}$ and $m_{i} \leqslant 18.0$ & $53(3)$ \\
2 & $3.0 \leqslant z_{\text {reg }} \leqslant 3.5$ and $m_{i} \leqslant 18.0$ & $49(5)$ \\
3 & $\left(2.5 \leqslant z_{\text {reg }} \leqslant 3.0\right.$ and $\left.m_{i} \leqslant 18.0\right)$ & $122(12)$ \\
& OR $\left(3.5 \leqslant z_{\text {reg }}\right.$ and $\left.18.0<m_{i} \leqslant 18.5\right)$ & \\
4 & $3.0 \leqslant z_{\text {reg }} \leqslant 3.5$ and $18.0<m_{i} \leqslant 18.5$ & $59(8)$ \\
5 & $2.5 \leqslant z_{\text {reg }} \leqslant 3.0$ and $18.0<m_{i} \leqslant 18.5$ & $46(16)$ \\
10 & all the remaining candidates & 5 \\
\hline
\end{tabular}

Spectroscopic observations could successfully identify 290 good PS-ELQS candidates, of which 190 are quasars at $z \geqslant 2.8$. A total of 44 good PS-ELQS candidates have not yet been observed, but are targeted in future observing campaigns. A list of the remaining PS-ELQS candidates is given in Table 10 in Appendix D.

\section{Spectroscopic Follow-up Observations and Data Reduction}

Dedicated observational campaigns for the PS-ELQS began in fall 2017 after the candidate selection was frozen in. Observations were completed in 2018 June. In this section we will describe the observational setups and the data reduction procedure.

\subsection{SOAR}

The focus of this quasar survey was the mostly unexplored area of the PS1 $3 \pi$ survey between Galactic latitudes of $-30<b<0$. Therefore the majority of our observations were carried out with the Goodman High Throughput Spectrograph (Goodman HTS; Clemens et al. 2004) on the Southern Astrophysical Research (SOAR) Telescope $(4.1 \mathrm{~m})$. Spectra were taken in 2017 October 6-10, 2018 January 22-24, 2018 April 4-6, and 2018 June 2-4. We used the $400 \mathrm{~g} \mathrm{~mm}^{-1}$ grating with central wavelengths of 6000 and $7300 \AA$. The first setup utilized the GG-385 blocking filter, whereas we used the GG-495 blocking filter for the second setup. The spectra have a wavelength coverage of $\sim 4000-8000$ and $\sim 5300-9300 \AA$ for the two different central wavelengths. We chose slit widths of 1 "! 0 or 1 "! 2 dependent on the weather conditions, resulting in spectral resolutions of $R \approx 830$ and $R \approx 690$, respectively. Exposure times varied between 3 and 15 minutes depending on the target magnitude and the atmospheric transparency.

\subsection{VATT Observations}

Identification spectroscopy in the northern hemisphere was carried out with the VATTSpec spectrograph on the Vatican Advanced Technology Telescope (VATT). Using the $300 \mathrm{~g} \mathrm{~mm}^{-1}$ grating blazed at $5000 \AA$ in first order, we achieved a resolution of $R \sim 1000$ (1"15 slit) and a coverage of $\sim 4000 \AA$ around our chosen central wavelength of $\sim 5850 \AA$. Targeted PS-ELQS observations were conducted in 2017 November 7-12, 2018 March 19-21, and 2018 May 17-18. Depending on the source and the conditions the exposure times varied between 15 and 30 minutes.

\subsection{MMT Observations}

We followed up our newly discovered quasars with the Red Channel Spectrograph on the MMT. We used the $300 \mathrm{~g} \mathrm{~mm}^{-1}$ grating blazed at $1 \mathrm{st} / 4800 \AA$ with central wavelengths of 5655 , 5570, and $5900 \AA$. The grating has an approximate coverage of $3310 \AA$ and achieves a resolution of $R \approx 400$ to 300 for the 1 ". 25 and the 1 ". 5 slits. Depending on the source and the weather conditions we chose exposure times of $\sim 3-15$ minutes per spectrum. Observations were taken in 2017 October 20-21, 2017 November 16, and 2018 May 14.

\subsection{Data Reduction}

We reduced the spectra with the long-slit reduction methods within the IRAF software package (Tody 1986, 1993). Standard bias subtraction, flat-field correction, and sky subtraction were applied. Sky subtraction and spectral extraction were done using the apall routine with optimal extraction (weights = variance) and cosmic ray reduction. The resulting low- to medium-S/N spectra allowed quasars to be easily identified by their broad emission lines. The wavelength calibration was based on internal lamps, and spectral fluxes were initially calibrated using at least one spectrophotometric standard star per night. However, changing weather conditions did not allow for absolute flux calibration and we therefore scaled the fluxes to match the observed PS1 $r$-band magnitudes. The spectra were not corrected for telluric absorption features.

\section{The PS-ELQS Quasar Catalog}

We conducted spectroscopic follow-up observations for 290 of our 334 good PS-ELQS candidates. We discovered a total of 218 new quasars, of which 190 are at $z \geqslant 2.8$. The resulting PS-ELQS quasar catalog includes a total of 592 quasars at $z \geqslant 2.8$ :

1. 285 quasars from DR14Q

2. 190 newly discovered quasars (PS-ELQS)

3. 54 quasars from ELQS

4. 47 quasars from MQC

5. 13 quasars from J. Yang et al. (2019, in preparation)

6. three quasars from DR7Q. 


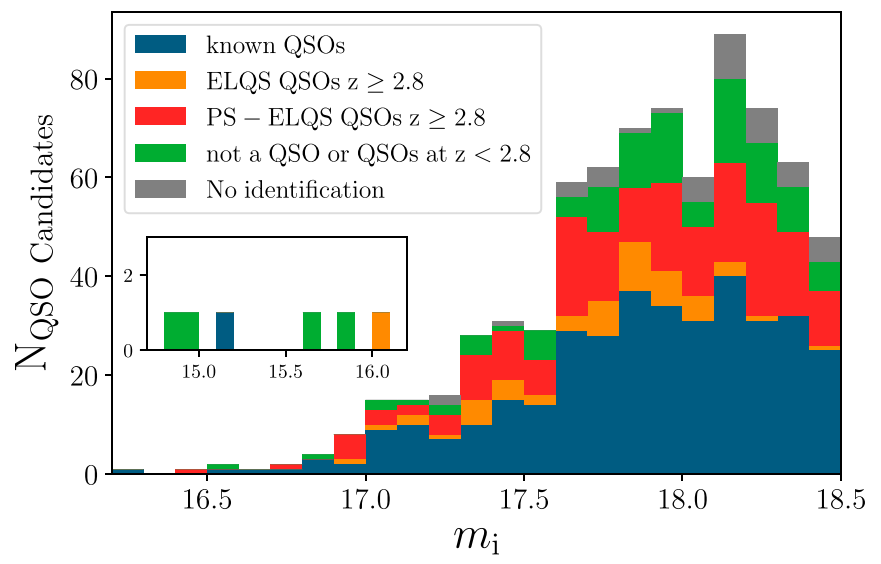

Figure 6. Histogram of the spectroscopic completeness of all good PS-ELQS candidates as a function of their dereddened PS1 $i$-band magnitude $m_{i}$. Dark blue and orange colors indicate all candidates from the general literature and the ELQS survey at $z \geqslant 2.8$. All newly identified candidates in this work are highlighted in red (QSOs at $z \geqslant 2.8$ ) and green (QSOs at $z<2.8$ or not a QSO). All candidates that remain unidentified are depicted in gray.

Excluding the 40 candidates with unreliable photometry we could identify 592 quasars at $z \geqslant 2.8$ out of 766 good candidates $(=806-40)$, of which 44 have not been observed. Therefore we calculate a minimum selection efficiency $77 \%$ (592/766) for the PS-ELQS quasar selection. For quasars at all redshifts our selection efficiency reaches $\sim 85 \%(650 / 766)$. Out of the 72 objects which were identified not to be quasars at $z \geqslant 2.8$, we classified 36 as stars (K: 18, M: seven, G: two, F: two, one cataclysmic variable, and six unidentified stars) and 14 as galaxies. The remaining 22 objects are not quasars at $z \geqslant 2.8$, but have often too low $\mathrm{S} / \mathrm{N}$ to classify them with certainty as a star, galaxy, or low-redshift quasar. The random forest classification results on the validation set (see Figure 3) suggest a much higher efficiency of $99 \%$ for quasars at any redshift. The discrepancy with the much lower observed efficiency of $85 \%$ is likely to originate in differences between the training set and the photometric sample. As we do not apply the $J K W 2$ color cut to the stars' training set, because it results in an undersized sample size (314 objects), the distribution of stellar types in the training set is different from the photometric candidate sample. For example, $42 \%$ of stars in the training set are $\mathrm{K}$ and $\mathrm{M}$ stars, while this ratio increases to $69 \%$ once the $J K W 2$ color cut is applied to the training set. Therefore, the photometric candidate sample has likely a higher fraction of contaminants that can mimic quasars at $z>2.8$, decreasing our overall efficiency.

The full PS-ELQS quasar catalog is published alongside this work in digital form. It also includes information on matches to Galaxy Evolution Explorer (GALEX), ROSAT 2RXS, and XMMSL2 (see Section 7.3 for details). Table 7 in Appendix A provides an overview over all the columns.

Figure 6 shows a histogram of all good candidates in the PSELQS sample as a function of their dereddened PS1 $i$-band magnitude, $m_{i}$. Known quasars at $z \geqslant 2.8$ in the literature are colored dark blue, excluding the known objects published as part of the ELQS (orange). All 190 newly identified quasars at $z \geqslant 2.8$ are shown in red, while all lower-redshift quasars and non-quasars are shown in green. The remaining 44 good candidates are highlighted in gray.

We present the discovery spectra of all newly identified quasars at $z \geqslant 2.8$ in Figures 9-11 in Appendix B. Additional identification spectra for new lower-redshift quasars are shown in Figure 12. All discovery spectra are sorted by redshift, beginning with the lowest-redshift spectrum. A dark blue, orange, and red colored bar at the top of each spectrum indicates the broad Ly $\alpha, \mathrm{Si}$ IV and C IV emission lines at 1215, 1400, $1549 \AA$ (rest-frame). Strong artifacts, like cosmic rays, are removed using an iterative sigma-clipping procedure. The spectra are not smoothed and spectral fluxes are scaled according to the PS1 $r$-band photometric measurement. Redshifts for all newly discovered quasars are measured by visual comparison to a redshifted quasar template spectrum (Vanden Berk et al. 2001). We estimate this method to have a redshift uncertainty of $\Delta z \approx 0.02$.

We calculate absolute magnitudes at $1450 \AA$ rest-frame, $M_{1450}$, from the dereddened PS1 $i$-band magnitudes for all quasars. This transformation includes a $k$-correction term which we estimate using a large grid of simulated quasar spectra. The simulated quasar spectra and synthetic PS1 magnitudes are generated by the simqs ${ }^{9}$ package (McGreer et al. 2013). The code begins by building a quasar continuum from a number of specified power-law slopes. Broad and narrow emission lines are then added onto the continuum as well as Fe emission and an IGM absorption model for the $\operatorname{Ly} \alpha$-forest. Our model does not account for intrinsic extinction from the quasar host galaxy, as the survey is targeted at the unobscured UV-bright quasar population. We adopt the values of Schindler et al. (2018) to build the spectral model and calculate a large grid of quasars with 28 cells over $m_{i}=14-18$ and 53 cells across $z=0.2-5.5$ with a total of 200 model spectra per cell. $K$-corrections are then calculated for each grid cell and interpolated to retrieve individual $k$-corrections for all newly identified quasars.

Figure 7 shows all 592 quasars of the PS-ELQS quasar catalog as a function of their absolute magnitudes, $M_{1450}$, and redshifts, $z$. Known quasars from the literature are divided into objects from the SDSS quasar catalogs (dark blue dots), sources from the MQC and J. Yang et al. (2019, in preparation, green triangles), and the ELQS quasar catalog (orange circles). Newly discovered quasars identified with this work are highlighted as red diamonds. The black solid line shows the faint-end magnitude limit of $m_{i}=18.5$ converted into $M_{1450}$. Histograms depict the distributions along each axis.

We provide a list of all newly discovered quasars within the PS-ELQS quasar catalog $(z \geqslant 2.8)$ in Table 8 in Appendix B. It contains the position in equatorial coordinates, the PS1 $i$-band magnitude, the absolute magnitude at $1450 \AA$, the determined spectroscopic redshift, near- and far-UV magnitudes from GALEX GR 6/7, and further notes. We provide the same information for all newly discovered quasars at $z<2.8$ in Table 9 in Appendix C.

\subsection{Quasar Discoveries at Low Declinations}

The PS1 $3 \pi$ survey covers $3 \pi$ sr of the northern sky, including the entire SDSS footprint. Therefore the PS-ELQS extends the efforts of the SDSS quasar surveys to a new region of $\sim 9600 \mathrm{deg}^{2}$. The majority of that area $\left(\sim 5600 \mathrm{deg}^{2}\right)$ is at lower declinations $($ decl. $=30-0)$. The PS-ELQS quasar catalog contains 207 quasars at lower declinations, of which only 70 were known before. Therefore we approximately treble the known population of luminous intermediate-redshift quasars in this region.

\footnotetext{
https://github.com/imcgreer/simqso
} 


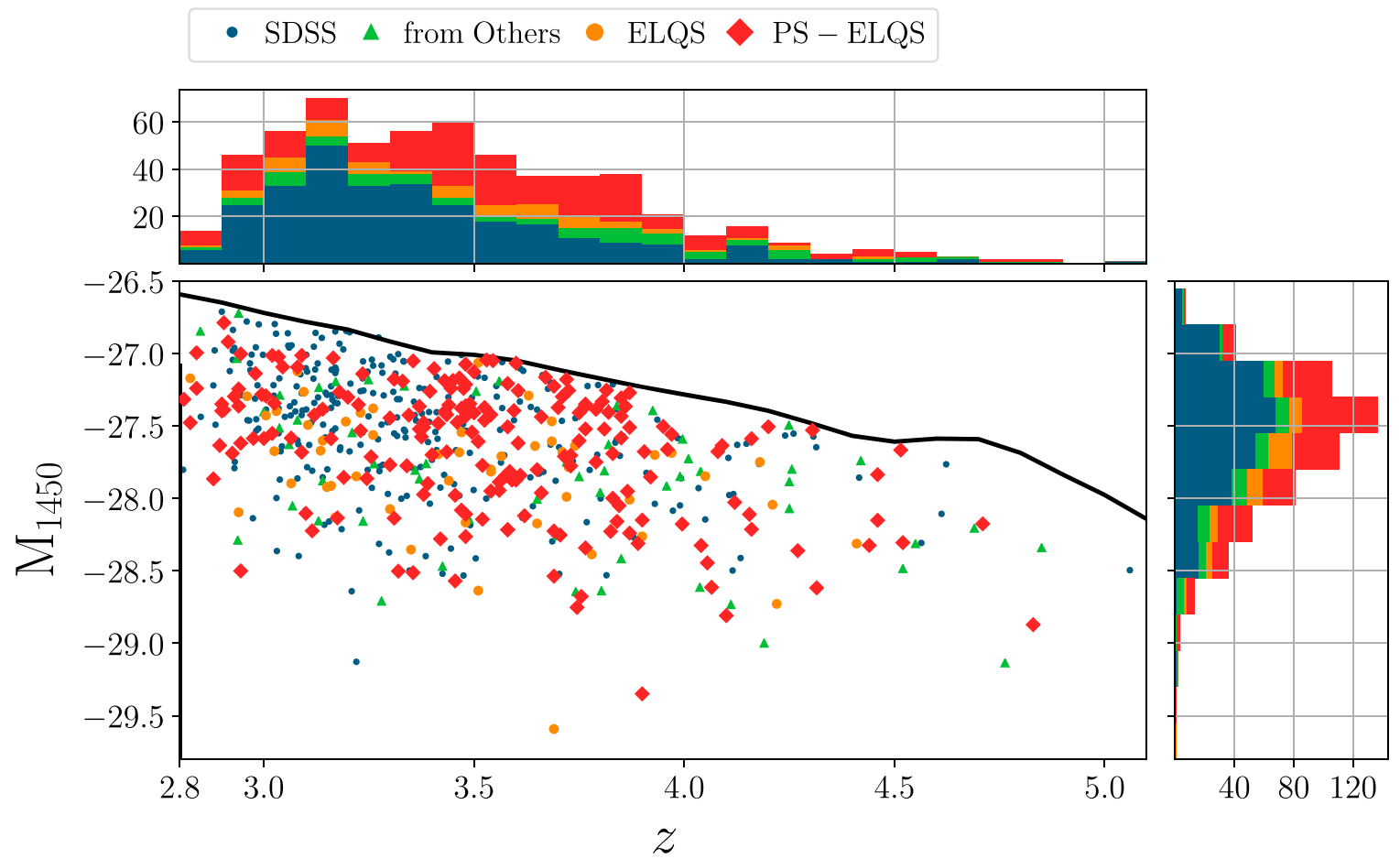

Figure 7. All quasars in the PS-ELQS quasar catalog in the absolute magnitude $\left(M_{1450}\right)$ and redshift $(z)$ plane. Quasars identified in the literature are shown as dark blue dots (SDSS DR7Q/DR14Q), green triangles (other sources), and small orange circles (ELQS). Newly identified quasars by the PS-ELQS are depicted as larger red diamonds. The solid black line shows our limit on the apparent PS1 $i$-band magnitude $\left(m_{i}=18.5\right)$. The histograms summarize the distribution of sources along their respective axis.

\subsection{Notes on Broad Absorption Line Quasars}

As part of the PS-ELQS we discovered a range of broad absorption line (BAL) quasars. Some of these objects have low-ionization BALs (LoBALs), showing broad absorption troughs from $\mathrm{Mg}$ II, and some of them also show absorption from meta-stable Fe II (FeLoBALs). We provide notes on the redshift identification and the absorption properties for some BAL quasars below, where the redshift measurement from the broad emission lines is difficult due to the absorption features. This is not an exhaustive list of all PS-ELQS quasars with BAL absorption features.

\subsubsection{J003856.98-292224.3}

This quasar is likely a FeLoBAL at $z \approx 2.27 \pm 0.005$ with strong nitrogen emission. The peak at $\sim 4150 \AA$ is $\mathrm{N} \mathrm{V}$ in the observed frame and the narrower line at $5750 \AA$ is N III] at $1750 \AA$ rest-frame. BAL troughs are seen in N V, C II, Si IV, C IV, Al II, Al III, and Fe II.

\subsubsection{J021119.80-195943.0}

J021119.80-195943.0 is a FeLoBAL at $z=2.45 \pm 0.02$. The emission redshift is uncertain, because even the $1900 \AA$ complex is affected by absorption. It also displays unusually strong N III] $1750 \AA$ emission. The red edges of the troughs are around $z=2.365$.

\subsection{3. $J 023500.45+023829.2$}

This BAL quasars has a redshift of $z=1.975 \pm 0.005$, primarily identified by the $1900 \AA$ complex. There is a C IV mini-BAL at $z=1.96$ and a C IV BAL at $z=1.85$.

\subsubsection{J033559.99-132601.8}

This LoBAL can be identified to be at $z=1.900 \pm 0.005$ from the narrow $\mathrm{C}$ IV and $\mathrm{Al}$ III emission. It displays absorption in $\mathrm{C}$ IV, Al III, and Mg II.

\subsubsection{J044756.84-230748.3}

Based on multiple absorption lines arising from a nearby damped Ly $\alpha$ absorber, this quasar has a redshift of at least $z=$ $2.945 \pm 0.005$. It displays weak broad C IV emission from 5700 $6300 \AA$ and weak broad $1900 \AA$ emission around 7100-7600 $\mathrm{A}$. It has a LoBAL with Si IV, C IV, and Al III at $z=2.82$ as well as a mini-BAL in N V, Si IV, and C IV at $z=2.745$.

\subsubsection{J113252.86-063243.3}

$\mathrm{J} 113252.86-063243.3$ is likely at $z=2.41 \pm 0.01$, mainly identified from the $1900 \AA$ emission complex. It displays a LoBAL (Si IV, C IV, Al II, Al III) at $z=2.27$. It further has narrower absorption troughs (Si IV, C IV, Al II, Al III) at $z=2.319, z=2.333$, and $z=2.363$.

\subsection{7. $J 191946.08+743747.1$}

This BAL quasar is at $z=1.604 \pm 0.001$ identified by the narrow Mg II emission. It shows a FeLoBAL with Al II, Al III, Fe III, Fe II, and Mg II absorption. The red end of the troughs is around $z=1.55$. It further displays narrow absorption in Al II, Al III, Fe II, and Mg II at $z=1.5915 \pm 0.0005$.

\subsubsection{J220912.01+061920.1}

This quasar is at $z=1.91 \pm 0.01$, identified by the narrow $\mathrm{C}$ IV and Al III lines. It displays a FeLoBAL (Si IV, C IV, Al II, Al III, Fe III, Mg II), where the blue end of the troughs is around 
$z=1.76$. It further has narrower Si IV, C IV, $\mathrm{Al} \mathrm{II,} \mathrm{Al}$ III, and Fe II absorption around $z=1.893$.

\subsection{Cross-matches to GALEX, ROSAT $2 R X S$, and XMMSL2}

To obtain near- and far-UV photometry, we cross-matched the PS-ELQS quasar catalog with the GALEX GR6/7 Data Release (Martin et al. 2005) within 2!"0. We obtained the available photometry in the far- and near-UV bands at

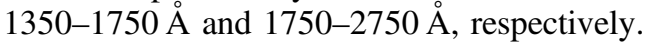

We could identify a total of 49 matches to the full PS-ELQS catalog, of which 17 were detections in both bands, three only in the far-UV band, and 29 only in the near-UV band. Of all 190 newly discovered quasars four have detections in both GALEX bands and another seven are detected in the near-UV. The detections could potentially signal that the quasars' flux has not been fully absorbed by neutral hydrogen along the line of sight and thus these objects are likely promising targets for studies of He-reionization (e.g., Worseck et al. 2016).

In addition, we utilize the AllWISE counterparts to X-ray detections (Salvato et al. 2018) from the ROSAT (Truemper 1982) reprocessed 2RXS catalog (Boller et al. 2016) and the XMM Newton Slew 2 Survey (XMMSL2) to match the PSELQS quasar catalog with X-ray sources. We have matched the AllWISE positions of our sources to the AllWISE positions of the counterpart catalogs within a 1 !"0 aperture.

There are a total of 12 matches between PS-ELQS and ROSAT 2RXS and three between PS-ELQS and XMMSL2.

Of the newly discovered quasars, one, J124615.10+ 713923.6 at $z=3.995$, has an X-ray counterpart in ROSAT 2RXS with $f_{0.1-2.4 \mathrm{keV}}=2.26 \times 10^{-13} \mathrm{erg} \mathrm{cm}^{-2} \mathrm{~s}^{-1}$ at a distance of 62 ". 7 . As the match distance implies, this AllWISE source is not the most probable match to the X-ray source (TRXS_match_flag $=2$ ) with a $p_{i} \approx 0.24$ relative probability to be the correct counterpart to the X-ray source among all possible AllWISE candidates. While we wanted to report this here for completeness, we would also like to caution against blindly associating the X-ray flux with the quasar.

Another new PS-ELQS quasar, J095947.52-103437.7 ( $z=$ 3.165), has a counterpart in XMMSL2. We further want to report on an XMMSL2 counterpart for the quasar J171721.32 $+422428.3(z=3.495)$. The latter quasar was discovered during pilot observations of the ELQS (Schindler et al. 2018), but not selected to be in the ELQS quasar catalog. In both cases the AllWISE counterparts constitute the best match to the X-ray source and seem reliable. The counterpart to J095947.52-103437.7 has a $0.24 \times 10^{-12} \mathrm{erg} \mathrm{cm}^{-2} \mathrm{~s}^{-1}$ detection in the soft band and a flux of $0.67 \times 10^{-12} \mathrm{erg} \mathrm{cm}^{-2} \mathrm{~s}^{-1}$ in the total band. The X-ray counterpart to J171721.32+422428.3 was detected in the soft band with $0.94 \times 10^{-12} \mathrm{erg} \mathrm{cm}^{-2} \mathrm{~s}^{-1}$ and in the total band with $2.42 \times 10^{-12} \mathrm{erg} \mathrm{cm}^{-2} \mathrm{~s}^{-1}$.

Information on all GALEX, ROSAT 2RXS, and XMMSL2 matches is included in the full PS-ELQS quasar catalog (see Table 7 in Appendix A).

\section{Discussion}

\subsection{PS-ELQS Completeness Estimates}

The goal of the PS-ELQS was to extend the novel quasar selection method of the ELQS to a much larger area. However, the final PS-ELQS quasar catalog includes only 343 quasars with $m_{i} \leqslant 18.0$ at $z \geqslant 2.8$ compared to the 407 quasars in the ELQS quasar catalog. A further comparison to the DR14Q showed that we only recover roughly $56 \%(71 \%, 89 \%)$ of the bright $\left(m_{i} \leqslant 18.0\right)$ quasars at at $z \geqslant 2.8(z \geqslant 3.0, z \geqslant 3.5)$, indicating that the PS-ELQS missed quasars at $z \leqslant 3.5$ compared to the ELQS.

In order to understand this difference and characterize the biases in our selection we calculated the PS-ELQS completeness for the random forest classification and redshift estimation. The selection function is estimated based on the sample of simulated quasars that were used to determine the $k$-correction described in Section 7.

The PS-ELQS completeness of the quasar selection based on the random forest classification is shown in panel (a) of Figure 8. The results are generally independent of the $i$-band magnitude, but show clear features as a function of redshift. There are two redshift ranges, $z \approx 3.2-3.5$ and $z \approx 3.7-4.0$, where the completeness drops to $75 \%-85 \%$. In the absence of $u$-band photometry these redshift ranges can be associated with stellar confusion. At $z \geqslant 5$ our completeness drops steeply, analogous to ELQS2. The absence of quasars at these redshifts and magnitudes in the training set results in incorrect classifications.

Panel (b) of Figure 8 shows the selection function resulting from the redshift selection based on the random forest regression (photometric redshift estimation). While we should select all quasars with $z_{\text {reg }} \geqslant 2.8$, we miss the majority $(>50 \%)$ of sources between $z=2.8$ and $z=3.5$. This effect clearly explains why the PS-ELQS missed many known quasars at $z \leqslant 3.5$, which were selected by the original ELQS.

As the main difference of the PS-ELQS to the ELQS is the optical photometry, we suspect that our decreased completeness at lower redshifts is a result of the missing $u$-band photometry in PS1. In order to test this, we add the SDSS $u$-band photometry to the number of features for the random forest and rerun our completeness calculation. The selection functions for the re-run classification and redshift selections are displayed in panels (c) and (d) of Figure 8. The results clearly confirm our suspicion. Comparing panels (a) and (c) highlights how adding the $u$-band visibly improves the classification selection at $z \approx 3.2-3.5$ and $z \approx 3.7-4.0$. Furthermore, panels (b) and (d) emphasize that the missing $u$-band in PS1 is the cause of the low completeness at $z \leqslant 3.5$ for PS-ELQS. A second look at the comparison between photometric redshifts and spectroscopic values in Figure 4 shows that the quasars at $z \geqslant 2.5$ are predominantly found below the $z_{\text {spec }}=z_{\text {reg }}$ diagonal line. This translates into a bias on our photometric redshift estimates toward lower values, which in turn causes the low completeness in the redshift selection between $z=2.8$ and $z=3.5$.

\subsection{Applications for the PS-ELQS Quasar Sample}

In spite of the shortcomings of the PS-ELQS selection due to the missing $u$-band photometry of PS1, the PS-ELQS provides the most complete sample of extremely luminous quasars at $z=3.5-5.0$. Our survey covered around $21,486 \mathrm{deg}^{2}$, making it the largest homogeneous spectroscopic quasar survey at these redshifts. We have successfully discovered 190 new quasars at $z \geqslant 2.8$, tripling the known quasars at decl. $\leqslant 0$ in the PS-ELQS quasar catalog.

Our area coverage and the high completeness at $z=3.5-5.0$ makes the PS-ELQS quasar sample uniquely equipped to constrain the volume density of extremely luminous quasars at intermediate redshifts. This is our first science goal, once the remaining 44 quasar candidates are observed to guarantee spectroscopic completeness. 


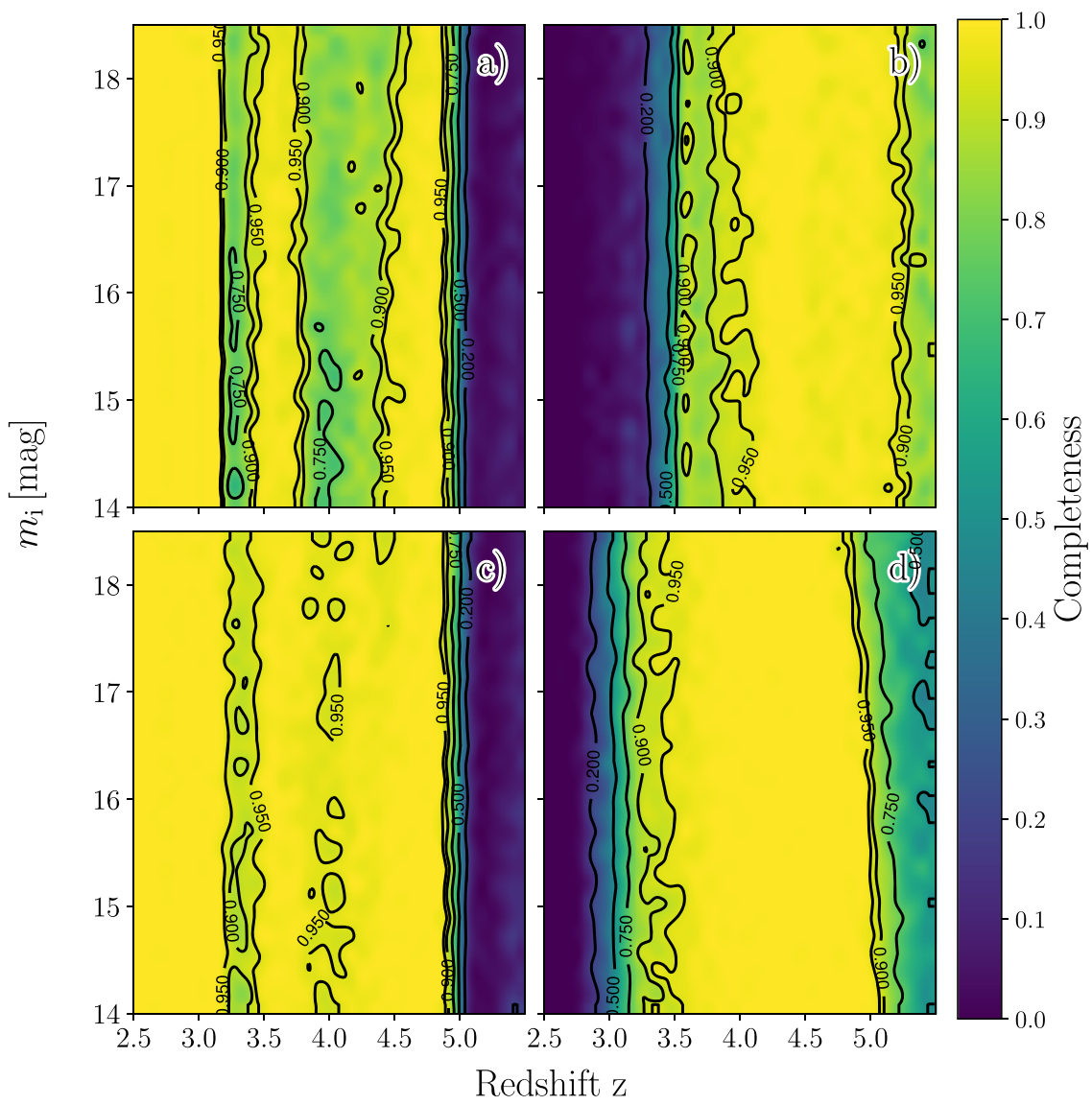

Figure 8. PS-ELQS completeness estimates as a function of redshift and $i$-band magnitude. The completeness maps were determined by the fraction of simulated quasars, selected by our different criteria, to all simulated quasars. Panel (a): Completeness of the PS-ELQS classification selection; panel (b): completeness of the PSELQS photometric redshift selection; panel (c): completeness of the classification selection (including the SDSS $u$-band); panel (d): completeness of the photometric redshift selection (including the SDSS $u$-band). Contour levels are drawn with solid lines at 20\%, 50\%, 75\%, 90\%, and 95\% completeness.

Table 6

Possible Quasar Associations Identified from the PS-ELQS Sample

\begin{tabular}{|c|c|c|c|c|c|}
\hline AllWISE Designation & $\begin{array}{l}\text { R.A. (J2000) } \\
\text { (hh:mm:ss.sss) }\end{array}$ & $\begin{array}{l}\text { Decl. (J2000) } \\
\text { (dd:mm:ss.ss) }\end{array}$ & $z$ & $\begin{array}{l}\text { Separation Angle } \\
\text { (arcmin) }\end{array}$ & $\begin{array}{l}\text { Proper 3D Distance } \\
\left(h^{-1} \mathrm{Mpc}\right)\end{array}$ \\
\hline J030341.04-002321.8 & 03:03:41.045 & $-00: 23: 21.87$ & 3.227 & $\ldots$ & $\cdots$ \\
\hline J030449.85-000813.4 & 03:04:49.859 & $-00: 08: 13.54$ & 3.295 & 22.92 & 12.30 \\
\hline J091647.60-113009.9 & 09:16:47.616 & $-11: 30: 09.91$ & 3.870 & $\cdots$ & $\cdots$ \\
\hline J091746.54-115331.9 & $09: 17: 46.542$ & $-11: 53: 31.89$ & 3.920 & 27.46 & 9.63 \\
\hline $\mathrm{J} 102000.80-121151.4$ & 10:20:00.800 & $-12: 11: 51.45$ & 3.715 & $\cdots$ & $\cdots$ \\
\hline J172237.85+385951.9 & $17: 22: 37.854$ & $+38: 59: 51.87$ & 3.359 & $\cdots$ & $\cdots$ \\
\hline $\mathrm{J} 172338.84+392621.4$ & $17: 23: 38.809$ & $+39: 26: 21.38$ & 3.465 & 29.00 & 16.84 \\
\hline $\mathrm{J} 235054.64+200938.6$ & $23: 50: 54.634$ & $+20: 09: 38.62$ & 3.170 & $\ldots$ & $\cdots$ \\
\hline $\mathrm{J} 235201.30+200902.3$ & 23:52:01.307 & $+20: 09: 02.47$ & 3.087 & 15.66 & 13.70 \\
\hline $\mathrm{J} 230432.31-124819.6$ & 23:04:32.312 & $-12: 48: 19.64$ & 3.850 & $\ldots$ & $\ldots$ \\
\hline
\end{tabular}

Four of our newly discovered quasars at $z=2.900,2.905$, 2.905 , and 3.320 have far- and near-UV fluxes as measured by GALEX. We have visually confirmed these detections in GALEX photometry. These objects provide promising targets to investigate the $\mathrm{He}$ reionization of the universe. In general, the PS-ELQS quasars' strong flux facilitates efficient high-resolution spectroscopy to characterize the $\operatorname{Ly} \alpha$ forest and intervening absorption systems.

From the PS-ELQS quasar catalog we have already identified five potential quasar pairs with angular separations of $\leqslant 30 \mathrm{arcmin}$ and proper distances of $\leqslant 20 h^{-1} \mathrm{Mpc}$ as well as an association of three quasars with larger angular distances but 
similar redshifts. We provide details for all of these objects in Table 6. The relative distances are always measured with respect to the first object in the pair or association.

These quasar associations demonstrate the value of the PSELQS sample for quasar clustering measurements. Previous measurements on quasar clustering (e.g., Myers et al. 2006, 2007; Shen et al. 2007; da Ângela et al. 2008; Ross et al. 2009; White et al. 2012; Eftekharzadeh et al. 2015; Rodríguez-Torres et al. 2017; Timlin et al. 2018) have shown that these populations show a high level of clustering and that quasars are inferred to reside in dark matter haloes of $10^{12} h^{-1} M_{\odot}$ at most redshifts. Due to its size the PS-ELQS quasar sample will only allow for a very sparse measurement; however, the number of quasars should allow to constrain the minimum dark matter halo mass for this extremely luminous population at $z \sim 3.8$. In combination with the measurement of the ELQS quasar luminosity function (Schindler et al. 2019), one will be able to place constraints on the duty cycle of these rare objects.

\section{Conclusions}

We present the results of the PS-ELQS, a spectroscopic quasar survey covering $\sim 21,486 \mathrm{deg}^{2}$ of the $3 \pi$ PS1 footprint. We apply the successful quasar selection strategy of the ELQS survey in the SDSS footprint to this larger area using the PS1 photometry. The candidates are selected based on a $J K W 2$ color cut using 2MASS $J$ and $K_{s}$, and AllWISE $W 2$ photometry. Random forest photometric redshift estimation and classification on all PanSTARRS PS1 and AllWISE $W 1$ and $W 2$ photometric bands lead to a highly efficient $(\geqslant 77 \%)$ quasar selection.

We selected a total of 806 bright $\left(m_{i} \leqslant 18.5\right)$ quasar candidates with regression redshifts of $z_{\text {reg }} \geqslant 2.8$. After the exclusion of 428 sources known in the literature, of which 70 were identified with the ELQS survey, we rejected 40 candidates during visual inspection of their photometry.

We selected a total of 334 good candidates for spectroscopic follow-up observations and were able to observe 290 of the objects with the SOAR telescope, the MMT, and the VATT. We have discovered a total of 218 new quasars, of which 190 are at $z \geqslant 2.8$. Based on our quasar selection we have constructed the PS-ELQS quasar catalog with a total of 592 quasars, including the 190 newly discovered quasars at $z \geqslant 2.8$.

Estimates of the PS-ELQS completeness for the classification and photometric redshift selections show that the missing $u$-band photometry of PS1 lowers our completeness to select quasars at $z=2.8-3.5$. This effect explains the low numbers of $z<3.5$ quasars compared to the original ELQS selection. However, not accounting for the photometric completeness of PS1, the PS-ELQS general completeness at $z=3.5-5.0$ is consistently above $70 \%$. As a result, the PS-ELQS provides the most complete sample of extremely luminous quasars at $z=3.5-5.0$.

A range of scientific applications will be able to leverage the information of this sample. For example, it is uniquely equipped to constrain the volume density of intermediate-redshift, extremely luminous quasars, to provide insight into quasar clustering of this rare population, and to facilitate studies of the IGM.

We would like to the thank the anonymous referee for a thorough report with many supportive suggestions which helped to improve this paper.

J.-T.S., X.F., I.D.M., and J.Y. acknowledge support from NSF grant AST-1515115 and NASA ADAP grant NNX17AF28G.
K.C.L. acknowledges support from the U.S. National Science Foundation (NSF) under awards AST1715213 and AST-1312950 and through award SOSPA4-007 from the National Radio Astronomy Observatory (NRAO).

Based on observations obtained at the Southern Astrophysical Research (SOAR) telescope, which is a joint project of the Ministério da Ciência, Tecnologia, Inovações e Comunicações (MCTIC) do Brasil, the U.S. National Optical Astronomy Observatory (NOAO), the University of North Carolina at Chapel Hill (UNC), and Michigan State University (MSU).

Based on observations with the VATT: the Alice P. Lennon Telescope and the Thomas J. Bannan Astrophysics Facility.

Observations reported here were obtained at the MMT Observatory, a joint facility of the University of Arizona and the Smithsonian Institution.

This publication makes use of data products from the Two Micron All Sky Survey, which is a joint project of the University of Massachusetts and the Infrared Processing and Analysis Center/California Institute of Technology, funded by the National Aeronautics and Space Administration and the National Science Foundation.

This publication makes use of data products from the Widefield Infrared Survey Explorer, which is a joint project of the University of California, Los Angeles, and the Jet Propulsion Laboratory/California Institute of Technology, funded by the National Aeronautics and Space Administration.

The Pan-STARRS1 Surveys (PS1) and the PS1 public science archive have been made possible through contributions by the Institute for Astronomy, the University of Hawaii, the PanSTARRS Project Office, the Max Planck Society and its participating institutes, the Max Planck Institute for Astronomy, Heidelberg and the Max Planck Institute for Extraterrestrial Physics, Garching, The Johns Hopkins University, Durham University, the University of Edinburgh, the Queen's University Belfast, the Harvard-Smithsonian Center for Astrophysics, the Las Cumbres Observatory Global Telescope Network Incorporated, the National Central University of Taiwan, the Space Telescope Science Institute, the National Aeronautics and Space Administration under grant No. NNX08AR22G issued through the Planetary Science Division of the NASA Science Mission Directorate, the National Science Foundation grant No. AST1238877, the University of Maryland, Eotvos Lorand University (ELTE), the Los Alamos National Laboratory, and the Gordon and Betty Moore Foundation.

Funding for the Sloan Digital Sky Survey IV has been provided by the Alfred P. Sloan Foundation, the U.S. Department of Energy Office of Science, and the Participating Institutions. SDSS acknowledges support and resources from the Center for High-Performance Computing at the University of Utah. The SDSS website is www.sdss.org.

SDSS is managed by the Astrophysical Research Consortium for the Participating Institutions of the SDSS Collaboration including the Brazilian Participation Group, the Carnegie Institution for Science, Carnegie Mellon University, the Chilean Participation Group, the French Participation Group, HarvardSmithsonian Center for Astrophysics, Instituto de Astrofísica de Canarias, The Johns Hopkins University, Kavli Institute for the Physics and Mathematics of the Universe (IPMU)/University of Tokyo, Lawrence Berkeley National Laboratory, Leibniz Institut für Astrophysik Potsdam (AIP), Max-Planck-Institut für Astronomie (MPIA Heidelberg), Max-Planck-Institut für Astrophysik (MPA Garching), Max-Planck-Institut für Extraterrestrische 
Physik (MPE), National Astronomical Observatories of China, New Mexico State University, New York University, University of Notre Dame, Observatório Nacional/MCTI, The Ohio State University, Pennsylvania State University, Shanghai Astronomical Observatory, United Kingdom Participation Group, Universidad Nacional Autónoma de México, University of Arizona, University of Colorado Boulder, University of Oxford, University of Portsmouth, University of Utah, University of Virginia, University of Washington, University of Wisconsin, Vanderbilt University, and Yale University.

This research has made use of the NASA/IPAC Extragalactic Database (NED) which is operated by the Jet Propulsion Laboratory, California Institute of Technology, under contract with the National Aeronautics and Space Administration.
This research made use of Astropy, ${ }^{10}$ a communitydeveloped core Python package for Astronomy (Astropy Collaboration et al. 2013, 2018). In addition, python routines from scikit-learn (Pedregosa et al. 2011), SciPy (Jones et al. 2001), Matplotlib (Hunter 2007), and Pandas (McKinney 2011) were used in the quasar selection, data analysis and creation of the figures.

\section{Appendix A The PS-ELQS Quasar Catalog}

The PS-ELQS quasar catalog is available as a machinereadable table online. It has 56 columns, detailed in Table 7.

Table 7

Description of the Full PS-ELQS Quasar Catalog Table

\begin{tabular}{|c|c|c|c|}
\hline Column & Column Name & Unit & Description \\
\hline 1 & WISE & $\ldots$ & WISE AllWISE survey designation \\
\hline 2 & PS1-RAdeg & $\operatorname{deg}$ & PS1 R.A., decimal degrees (J2000) \\
\hline 3 & PS1-DEdeg & $\operatorname{deg}$ & PS1 Decl., decimal degrees (J2000) \\
\hline 4 & PS1-RAh & $\mathrm{h}$ & PS1 Hour of R.A. (J2000) \\
\hline 5 & PS1-RAm & $\mathrm{m}$ & PS1 Minute of R.A. (J2000) \\
\hline 6 & PS1-RAs & s & PS1 Second of R.A. (J2000) \\
\hline 7 & PS1-DE- & $\ldots$ & PS1 Sign of the Decl. (J2000) \\
\hline 8 & PS1-DEd & $\operatorname{deg}$ & PS1 Degree of Decl. (J2000) \\
\hline 9 & PS1-DEm & $\operatorname{arcmin}$ & PS1 Arcminute of Decl. (J2000) \\
\hline 10 & PS1-DEs & $\operatorname{arcsec}$ & PS1 Arcsecond of Decl. (J2000) \\
\hline 11 & ALLWISE-RAdeg & $\operatorname{deg}$ & ALLWISE R.A., decimal degrees (J2000) \\
\hline 12 & ALLWISE-DEdeg & deg & ALLWISE Decl., decimal degrees (J2000) \\
\hline 13 & Ref & $\ldots$ & Reference of the quasar classification \\
\hline 14 & z-Ref & $\ldots$ & Best redshift of the quasar according to the reference \\
\hline 15 & M1450 & mag & Absolute magnitude at $1450 \AA$ calculated using the k-correction determined for this work \\
\hline $16-25$ & [band]mag-[survey] & mag & $\begin{array}{l}\text { Dereddened AB magnitudes of the PS1 grizy, 2MASS jh } \mathrm{k}_{\mathrm{s}} \text { and WISE W1W2 bands } \\
\quad \text { (surveys = [PS,2M,WISE]; bands = [g,r,i,z,y],[J,H,K],[W1,W2]). }\end{array}$ \\
\hline $26-35$ & e_[band]mag-[survey] & mag & $1 \sigma$ uncertainties on the $\mathrm{AB}$ magnitudes. \\
\hline 36 & EBV & mag & $\mathrm{E}(\mathrm{B}-\mathrm{V})$ color excess \\
\hline 37 & GALEX-Match & $\ldots$ & Boolean to indicate successful matches with GALEX GR6/7 \\
\hline 38 & $G A L E X$-Dist & $\operatorname{arcsec}$ & Match distance, GALEX GR6/7 to PS1 DR1 \\
\hline 39 & GALEX-NUVmag & mag & GALEX near-UV flux in magnitudes \\
\hline 40 & e_GALEX-NUVmag & mag & Error on the GALEX near-UV flux \\
\hline 41 & GALEX-FUVmag & mag & GALEX far-UV flux in magnitudes \\
\hline 42 & e_GALEX-FUVmag & mag & Error on the GALEX far-UV flux \\
\hline 43 & TRXS-match & $\ldots$ & Boolean to indicate successful matches to the ROSAT 2RXS AllWISE counterparts \\
\hline 44 & TRXS-Dist & $\operatorname{arcsec}$ & $\begin{array}{l}\text { Match distance between the ELQS AllWISE position to the ROSAT 2RXS AllWISE position. The distance } \\
\text { values are often } 0 \text { or otherwise extremely small, because the positions match to numerical accuracy. }\end{array}$ \\
\hline 45 & f_TRXS-match & $\ldots$ & $\begin{array}{l}\text { A flag indicating the most probable AllWISE ROSAT 2RXS cross-match with } 1 \text {. This is the case for all } \\
\text { matched objects. }\end{array}$ \\
\hline 46 & TRXS-FLUX & $\operatorname{erg~cm}{ }^{-2} \mathrm{~s}^{-1}$ & 2RXS flux \\
\hline 47 & e_TRXS-FLUX & $\operatorname{erg~cm}{ }^{-2} \mathrm{~s}^{-1}$ & 2RXS flux error \\
\hline 48 & XMM-match & $\ldots$ & Boolean to indicate successful matches to the XMMSL2 AllWISE counterparts \\
\hline 49 & XMM-Dist & $\operatorname{arcsec}$ & Match distance between the ELQS AllWISE position to the XMMSL2 AllWISE counterparts \\
\hline 50 & f_XMM-match & $\ldots$ & $\begin{array}{l}\text { A flag indicating the most probable AllWISE XMMSL2 cross-match with } 1 \text {. This is the case for all } \\
\text { matched objects. }\end{array}$ \\
\hline 51 & XMM-FLUX-B8 & $10^{-12} \mathrm{erg} \mathrm{cm}^{-2} \mathrm{~s}^{-1}$ & Total band $(0.2-12.0 \mathrm{keV})$ flux \\
\hline 52 & XMM-FLUX-B7 & $10^{-12} \mathrm{erg} \mathrm{cm}^{-2} \mathrm{~s}^{-1}$ & Hard band $(2.0-12.0 \mathrm{keV})$ flux \\
\hline 53 & XMM-FLUX-B6 & $10^{-12} \mathrm{erg} \mathrm{cm}^{-2} \mathrm{~s}^{-1}$ & Soft band $(0.2-2.0 \mathrm{keV})$ flux \\
\hline 54 & e_XMM-FLUX-B8 & $10^{-12} \mathrm{erg} \mathrm{cm}^{-2} \mathrm{~s}^{-1}$ & Total band $(0.2-12.0 \mathrm{keV})$ flux error \\
\hline 55 & e_XMM-FLUX-B7 & $10^{-12} \mathrm{erg} \mathrm{cm}^{-2} \mathrm{~s}^{-1}$ & Hard band $(2.0-12.0 \mathrm{keV})$ flux error \\
\hline 56 & e_XMM-FLUX-B6 & $10^{-12} \mathrm{erg} \mathrm{cm}^{-2} \mathrm{~s}^{-1}$ & Soft band $(0.2-2.0 \mathrm{keV})$ flux error \\
\hline
\end{tabular}

(This table is available in its entirety in machine-readable form.)

\footnotetext{
${ }^{10}$ http://www.astropy.org
} 


\section{Appendix B}

\section{Newly Discovered QSOs at $z \geqslant \mathbf{2 . 8}$}

We present general properties of the 190 newly discovered PS-ELQS quasars in Table 8 . Their discovery spectra are shown in Figures 9-11.

Table 8

Newly Discovered Quasars at $z \geqslant 2.8$ in the PS-ELQS Sample

\begin{tabular}{|c|c|c|c|c|c|c|c|}
\hline $\begin{array}{l}\text { R.A. (J2000) } \\
\text { (hh:mm:ss.sss) }\end{array}$ & $\begin{array}{l}\text { Decl. (J2000) } \\
\text { (dd:mm:ss.ss) }\end{array}$ & $\begin{array}{c}m_{i} \\
(\mathrm{mag})\end{array}$ & $\begin{array}{l}M_{1450} \\
(\mathrm{mag})\end{array}$ & $\begin{array}{c}\text { Spectroscopic } \\
\text { Redshift }\end{array}$ & $\begin{array}{l}\text { Near-UV }^{\mathrm{a}} \\
(\mathrm{mag})\end{array}$ & $\begin{array}{c}\text { Far-UV } \\
(\mathrm{mag})\end{array}$ & Notes $^{\mathrm{b}}$ \\
\hline $00: 20: 27.082$ & $-18: 44: 00.97$ & $18.31 \pm 0.01$ & -27.35 & 3.765 & $\ldots$ & $\ldots$ & 171008 \\
\hline $00: 27: 25.651$ & $-26: 44: 32.00$ & $17.93 \pm 0.01$ & -27.29 & 3.005 & $\ldots$ & $\ldots$ & 171008 \\
\hline 00:43:46.841 & $-11: 17: 02.06$ & $17.41 \pm 0.00$ & -28.11 & 3.480 & $\ldots$ & $\cdots$ & 171007 \\
\hline 00:55:15.845 & $-14: 59: 15.50$ & $18.39 \pm 0.01$ & -27.50 & 4.200 & $\cdots$ & $\cdots$ & 171008 \\
\hline 01:02:48.769 & $-20: 07: 28.70$ & $18.32 \pm 0.01$ & -27.30 & 3.710 & $\ldots$ & $\ldots$ & 171010 \\
\hline 01:03:05.501 & $-24: 49: 25.25$ & $17.77 \pm 0.01$ & -27.95 & 3.865 & $\ldots$ & $\ldots$ & 180122 \\
\hline 01:03:18.075 & $-13: 05: 10.19$ & $17.22 \pm 0.00$ & -28.61 & 4.065 & $\ldots$ & $\ldots$ & 171007 \\
\hline 01:09:33.398 & $+38: 20: 15.82$ & $18.45 \pm 0.01$ & -27.18 & 3.720 & $\ldots$ & $\ldots$ & 171020 \\
\hline 01:18:52.261 & $-09: 40: 16.07$ & $17.98 \pm 0.00$ & -27.54 & 3.495 & $\ldots$ & $\ldots$ & 171008 \\
\hline 01:28:18.883 & $-09: 57: 00.44$ & $18.14 \pm 0.01$ & -27.52 & 3.765 & $\cdots$ & $\cdots$ & 171008 \\
\hline 01:29:48.978 & $-04: 21: 49.49$ & $18.48 \pm 0.00$ & -27.07 & 3.600 & $\cdots$ & $\ldots$ & 171010 \\
\hline 01:39:11.231 & $-02: 31: 33.65$ & $18.38 \pm 0.01$ & -27.23 & 3.690 & $\ldots$ & $\ldots$ & 171010 \\
\hline 01:40:46.361 & $+36: 41: 30.22$ & $18.25 \pm 0.02$ & -27.18 & 3.310 & $\ldots$ & $\ldots$ & 171021 \\
\hline 01:50:41.591 & $-25: 08: 46.35$ & $17.70 \pm 0.01$ & -27.87 & 3.600 & $\cdots$ & $\cdots$ & 171008 \\
\hline 01:51:06.839 & $-28: 39: 33.76$ & $17.87 \pm 0.00$ & -27.78 & 3.730 & $\ldots$ & $\ldots$ & 171007 \\
\hline 02:01:58.777 & $+37: 17: 45.47$ & $18.16 \pm 0.01$ & -27.68 & 4.080 & $\cdots$ & $\cdots$ & 171020 \\
\hline 02:08:25.254 & $+17: 05: 48.91$ & $17.66 \pm 0.01$ & -27.77 & 3.300 & $\ldots$ & $\ldots$ & 171010 \\
\hline 02:12:20.417 & $+09: 17: 49.15$ & $17.64 \pm 0.01$ & -27.59 & 3.000 & $\ldots$ & $\ldots$ & 171008 \\
\hline 02:14:21.635 & $+09: 04: 07.05$ & $17.61 \pm 0.01$ & -27.94 & 3.560 & $\ldots$ & $\ldots$ & 171009 \\
\hline 02:19:48.831 & $+34: 47: 19.63$ & $17.74 \pm 0.01$ & -27.59 & 3.160 & $\ldots$ & $\ldots$ & 171020 \\
\hline 02:21:23.915 & $-14: 16: 54.82$ & $17.80 \pm 0.01$ & -27.80 & 3.650 & $\ldots$ & $\ldots$ & 180124 \\
\hline 02:21:26.889 & $-28: 22: 51.31$ & $18.31 \pm 0.01$ & -27.21 & 3.480 & $\cdots$ & $\cdots$ & 171008 \\
\hline 02:23:25.100 & $+22: 12: 11.77$ & $18.43 \pm 0.01$ & -27.25 & 3.815 & $\ldots$ & $\ldots$ & 171021 \\
\hline $02: 28: 40.587$ & $+35: 26: 17.59$ & $17.52 \pm 0.01$ & -27.97 & 3.380 & $\ldots$ & $\ldots$ & 171020 \\
\hline $02: 31: 49.748$ & $-11: 15: 20.81$ & $18.32 \pm 0.00$ & -27.19 & 3.430 & $\ldots$ & $\ldots$ & 171010 \\
\hline 02:35:51.443 & $-17: 57: 25.67$ & $18.16 \pm 0.00$ & -27.39 & 3.595 & $\cdots$ & $\cdots$ & 171009 \\
\hline $02: 45: 26.449$ & $+37: 10: 07.34$ & $17.67 \pm 0.01$ & -27.89 & 3.560 & $\ldots$ & $\ldots$ & 171111 \\
\hline 02:49:32.661 & $+27: 59: 25.10$ & $18.22 \pm 0.01$ & -27.01 & 3.020 & $\cdots$ & $\cdots$ & 171116 \\
\hline 02:55:29.671 & $+12: 28: 26.46$ & $17.50 \pm 0.01$ & -28.24 & 3.870 & $\ldots$ & $\ldots$ & 171010 \\
\hline 02:57:21.095 & $+15: 33: 23.09$ & $17.30 \pm 0.01$ & -28.14 & 3.310 & $\ldots$ & $\ldots$ & 171010 \\
\hline 03:01:51.627 & $+12: 12: 04.58$ & $17.10 \pm 0.00$ & -28.54 & 3.690 & $\cdots$ & $\ldots$ & 171010 \\
\hline 03:05:17.924 & $-20: 56: 28.22$ & $18.11 \pm 0.01$ & -27.66 & 3.960 & $\cdots$ & $\ldots$ & 171007 \\
\hline 03:05:59.775 & $+24: 25: 07.32$ & $18.17 \pm 0.01$ & -27.52 & 3.810 & $\cdots$ & $\cdots$ & 171109 \\
\hline 03:25:09.436 & $+27: 12: 00.36$ & $18.34 \pm 0.01$ & -27.21 & 3.580 & $\cdots$ & $\cdots$ & 171111 \\
\hline 03:29:06.257 & $+20: 24: 57.82$ & $17.82 \pm 0.01$ & -27.53 & 3.230 & $\cdots$ & $\cdots$ & 171111 \\
\hline 03:31:36.931 & $+21: 29: 32.29$ & $17.48 \pm 0.01$ & -27.85 & 3.190 & $\cdots$ & $\ldots$ & 171109 \\
\hline 03:39:08.180 & $-15: 38: 21.18$ & $17.93 \pm 0.00$ & -27.75 & 3.790 & $\ldots$ & $\ldots$ & 171006 \\
\hline $03: 41: 18.143$ & $+02: 24: 37.30$ & $17.60 \pm 0.01$ & -27.68 & 3.090 & $\cdots$ & $\ldots$ & 171008 \\
\hline $03: 53: 14.885$ & $-25: 18: 14.85$ & $18.45 \pm 0.01$ & -27.53 & 4.305 & $\ldots$ & $\ldots$ & 171008 \\
\hline 03:55:50.316 & $-14: 56: 39.05$ & $18.28 \pm 0.01$ & -27.24 & 3.475 & $\ldots$ & $\cdots$ & 171010 \\
\hline 03:56:17.616 & $-12: 03: 09.63$ & $18.29 \pm 0.00$ & -27.36 & 3.765 & $\cdots$ & $\ldots$ & 171008 \\
\hline 03:58:11.141 & $+25: 04: 01.62$ & $18.24 \pm 0.01$ & -27.41 & 3.750 & $\ldots$ & $\ldots$ & 171111 \\
\hline 03:59:15.718 & $-07: 41: 42.13$ & $17.24 \pm 0.00$ & -28.28 & 3.420 & $\ldots$ & $\ldots$ & 171006 \\
\hline 03:59:22.959 & $-19: 11: 27.82$ & $17.87 \pm 0.01$ & -27.24 & 2.840 & $\cdots$ & $\ldots$ & 171009 \\
\hline 04:08:20.966 & $-03: 08: 29.58$ & $18.06 \pm 0.00$ & -27.60 & 3.750 & $\ldots$ & $\ldots$ & 171009 \\
\hline 04:09:14.876 & $-27: 56: 32.89$ & $17.95 \pm 0.00$ & -28.15 & 4.460 & $\ldots$ & $\ldots$ & 171006 \\
\hline 04:10:53.654 & $-07: 47: 44.82$ & $17.62 \pm 0.00$ & -27.59 & 2.975 & $\cdots$ & $\ldots$ & 171009 \\
\hline 04:11:02.077 & $-01: 35: 15.15$ & $17.64 \pm 0.01$ & -27.96 & 3.660 & $\ldots$ & $\ldots$ & 171006 \\
\hline 04:32:29.308 & $-19: 17: 17.82$ & $17.88 \pm 0.00$ & -27.30 & 2.930 & $\ldots$ & $\cdots$ & 171009 \\
\hline $04: 41: 32.015$ & $-10: 16: 34.27$ & $18.21 \pm 0.01$ & -27.56 & 3.970 & $\cdots$ & $\ldots$ & 171008 \\
\hline $04: 47: 56.843$ & $-23: 07: 48.29$ & $16.71 \pm 0.00$ & -28.50 & 2.945 & $\cdots$ & $\cdots$ & $171006^{\mathrm{c}}$ \\
\hline 04:53:16.580 & $-09: 30: 24.94$ & $18.39 \pm 0.00$ & -27.10 & 3.405 & $\ldots$ & $\cdots$ & 180124 \\
\hline 04:54:20.311 & $-00: 37: 31.84$ & $18.25 \pm 0.02$ & -26.92 & 2.915 & $\ldots$ & $\ldots$ & 180124 \\
\hline 04:59:50.110 & $+07: 28: 02.71$ & $18.09 \pm 0.01$ & -27.42 & 3.435 & $22.67 \pm 0.32$ & $\ldots$ & 180124 \\
\hline 05:00:15.026 & $-24: 39: 27.24$ & $17.92 \pm 0.00$ & -27.61 & 3.510 & $\ldots$ & $\ldots$ & 171007 \\
\hline 05:03:54.146 & $-06: 08: 25.04$ & $18.22 \pm 0.01$ & -27.02 & 3.035 & $\ldots$ & $\ldots$ & 180124 \\
\hline
\end{tabular}


Table 8

(Continued)

\begin{tabular}{|c|c|c|c|c|c|c|c|}
\hline $\begin{array}{l}\text { R.A. (J2000) } \\
\text { (hh:mm:ss.sss) }\end{array}$ & $\begin{array}{l}\text { Decl. (J2000) } \\
\text { (dd:mm:ss.ss) }\end{array}$ & $\begin{array}{c}m_{i} \\
(\mathrm{mag})\end{array}$ & $\begin{array}{l}M_{1450} \\
(\mathrm{mag})\end{array}$ & $\begin{array}{l}\text { Spectroscopic } \\
\text { Redshift }\end{array}$ & $\begin{array}{l}\text { Near-UV } \\
(\mathrm{mag})\end{array}$ & $\begin{array}{c}\text { Far-UV } \\
(\mathrm{mag})\end{array}$ & Notes $^{\mathrm{b}}$ \\
\hline 05:20:01.728 & $-20: 14: 40.59$ & $18.26 \pm 0.00$ & -27.50 & 3.950 & $\cdots$ & $\cdots$ & 171008 \\
\hline 05:21:36.923 & $-13: 39: 38.79$ & $17.60 \pm 0.00$ & -28.36 & 4.270 & $\cdots$ & $\cdots$ & 171006 \\
\hline 05:39:46.870 & $-20: 08: 41.86$ & $18.29 \pm 0.01$ & -27.38 & 3.790 & $\cdots$ & $\cdots$ & 171021 \\
\hline 08:18:24.472 & $+82: 06: 48.47$ & $17.35 \pm 0.01$ & -28.22 & 3.580 & $\ldots$ & $\ldots$ & 180517 \\
\hline 08:51:03.208 & $+13: 02: 53.32$ & $18.47 \pm 0.01$ & -27.04 & 3.530 & $\cdots$ & $\cdots$ & 180122 \\
\hline 09:16:47.616 & $-11: 30: 09.91$ & $18.44 \pm 0.01$ & -27.27 & 3.870 & $\cdots$ & $\cdots$ & 180122 \\
\hline 09:17:46.542 & $-11: 53: 31.89$ & $17.90 \pm 0.01$ & -27.85 & 3.920 & $\cdots$ & $\cdots$ & 171111 \\
\hline 09:19:23.109 & $-00: 52: 08.00$ & $17.56 \pm 0.00$ & -27.62 & 2.945 & $\cdots$ & $\cdots$ & 180122 \\
\hline 09:28:05.302 & $+28: 27: 19.72$ & $17.80 \pm 0.01$ & -27.70 & 3.400 & $21.48 \pm 0.23$ & $\cdots$ & 171109 \\
\hline 09:34:04.053 & $-11: 11: 25.13$ & $17.94 \pm 0.01$ & -27.62 & 3.605 & $\cdots$ & $\cdots$ & 180122 \\
\hline 09:35:42.696 & $-06: 51: 18.94$ & $17.50 \pm 0.01$ & -28.32 & 4.040 & $\ldots$ & $\ldots$ & 180122 \\
\hline 09:40:24.121 & $-03: 23: 04.13$ & $17.60 \pm 0.00$ & -28.15 & 3.900 & $\ldots$ & $\ldots$ & 180123 \\
\hline 09:50:34.733 & $-21: 02: 50.74$ & $18.16 \pm 0.01$ & -27.36 & 3.480 & $\cdots$ & $\cdots$ & 180124 \\
\hline 09:59:47.524 & $-10: 34: 37.50$ & $18.28 \pm 0.03$ & -27.03 & 3.165 & $21.81 \pm 0.28$ & $\cdots$ & 180123 \\
\hline 10:14:30.281 & $-04: 21: 40.32$ & $17.44 \pm 0.01$ & -28.31 & 3.890 & $\cdots$ & $\cdots$ & 180123 \\
\hline 10:15:29.367 & $-12: 13: 14.34$ & $17.06 \pm 0.00$ & -28.81 & 4.100 & $\ldots$ & $\cdots$ & 180123 \\
\hline 10:15:40.799 & $-03: 27: 47.25$ & $17.67 \pm 0.01$ & -28.05 & 3.845 & $\ldots$ & $\ldots$ & 180123 \\
\hline 10:15:44.118 & $-11: 09: 22.77$ & $17.56 \pm 0.00$ & -28.16 & 3.840 & $\cdots$ & $\cdots$ & 180123 \\
\hline 10:20:00.800 & $-12: 11: 51.45$ & $17.92 \pm 0.01$ & -27.71 & 3.715 & $\cdots$ & $\cdots$ & 180404 \\
\hline 10:21:26.131 & $-11: 56: 22.39$ & $18.43 \pm 0.01$ & -27.16 & 3.670 & $\cdots$ & $\cdots$ & 180405 \\
\hline 10:31:58.288 & $-21: 44: 07.40$ & $17.69 \pm 0.00$ & -27.87 & 3.590 & $\cdots$ & $\cdots$ & 180404 \\
\hline 10:41:38.997 & $-09: 44: 37.94$ & $18.22 \pm 0.02$ & -27.14 & 3.235 & $\cdots$ & $\cdots$ & 180321 \\
\hline 10:46:27.942 & $-23: 39: 17.54$ & $18.04 \pm 0.01$ & -27.51 & 3.580 & $\ldots$ & $\ldots$ & 180122 \\
\hline 10:47:13.545 & $-06: 45: 38.19$ & $18.42 \pm 0.01$ & -27.05 & 3.355 & $\cdots$ & $\cdots$ & 180405 \\
\hline 10:51:22.689 & $-06: 50: 47.84$ & $17.34 \pm 0.01$ & -28.34 & 3.765 & $\ldots$ & $\cdots$ & 180122 \\
\hline 10:53:53.499 & $+25: 31: 15.50$ & $18.39 \pm 0.01$ & -27.13 & 3.500 & $\cdots$ & $\cdots$ & 180321 \\
\hline 10:54:49.678 & $-17: 11: 07.39$ & $16.92 \pm 0.00$ & -28.75 & 3.745 & $20.68 \pm 0.10$ & $\cdots$ & 180122 \\
\hline 11:08:48.484 & $-10: 22: 07.31$ & $18.26 \pm 0.01$ & -27.84 & 4.460 & $\ldots$ & $\cdots$ & 180123 \\
\hline 11:10:54.687 & $-30: 11: 29.95$ & $17.38 \pm 0.00$ & -28.87 & 4.830 & $\ldots$ & $\cdots$ & $180122^{\mathrm{d}}$ \\
\hline 11:13:05.343 & $-21: 25: 40.65$ & $17.60 \pm 0.01$ & -27.90 & 3.390 & $\cdots$ & $\cdots$ & 180122 \\
\hline 11:13:34.586 & $-07: 50: 33.49$ & $18.15 \pm 0.01$ & -27.09 & 3.045 & $\ldots$ & $\cdots$ & 180405 \\
\hline 11:14:03.257 & $-05: 02: 35.09$ & $17.48 \pm 0.00$ & -28.23 & 3.825 & $\cdots$ & $\cdots$ & 180122 \\
\hline 11:14:28.309 & $-04: 09: 38.76$ & $18.27 \pm 0.02$ & -27.24 & 3.445 & $\cdots$ & $\cdots$ & 180405 \\
\hline 11:19:56.987 & $-19: 28: 32.42$ & $18.01 \pm 0.02$ & -27.69 & 3.830 & $\cdots$ & $\cdots$ & 180123 \\
\hline 11:29:39.605 & $-23: 33: 49.64$ & $17.28 \pm 0.00$ & -27.86 & 2.880 & $\ldots$ & $\ldots$ & 180123 \\
\hline 11:33:55.641 & $-23: 05: 24.38$ & $18.15 \pm 0.01$ & -27.44 & 3.660 & $\cdots$ & $\cdots$ & 180405 \\
\hline 11:44:17.308 & $-05: 45: 34.69$ & $18.11 \pm 0.01$ & -27.41 & 3.500 & $\ldots$ & $\cdots$ & 180405 \\
\hline 11:49:14.377 & $-15: 30: 43.93$ & $17.68 \pm 0.01$ & -28.21 & 4.160 & $\cdots$ & $\cdots$ & 180404 \\
\hline 11:56:32.386 & $-07: 21: 14.26$ & $18.37 \pm 0.01$ & -26.79 & 2.905 & $21.70 \pm 0.27$ & $21.73 \pm 0.49$ & 180405 \\
\hline 12:09:29.549 & $-05: 17: 37.06$ & $18.43 \pm 0.01$ & -27.07 & 3.480 & $\ldots$ & $\ldots$ & 180405 \\
\hline 12:10:16.802 & $+80: 56: 03.21$ & $18.17 \pm 0.04$ & -27.35 & 3.490 & $\cdots$ & $\cdots$ & 180514 \\
\hline 12:10:30.332 & $-09: 57: 25.39$ & $18.03 \pm 0.01$ & -27.30 & 3.200 & $\cdots$ & $\cdots$ & 180405 \\
\hline 12:30:10.034 & $-06: 33: 34.10$ & $17.97 \pm 0.01$ & -27.44 & 3.300 & $\ldots$ & $\cdots$ & 180404 \\
\hline 12:36:12.047 & $-11: 36: 00.62$ & $18.00 \pm 0.01$ & -27.35 & 3.225 & $\cdots$ & $\cdots$ & 180405 \\
\hline 12:46:10.755 & $+75: 17: 11.07$ & $17.40 \pm 0.00$ & -28.14 & 3.520 & $\cdots$ & $\cdots$ & 180517 \\
\hline 12:46:15.090 & $+71: 39: 23.60$ & $17.62 \pm 0.00$ & -28.18 & 3.995 & $\ldots$ & $\cdots$ & 180517 \\
\hline 12:58:50.976 & $-18: 54: 30.55$ & $17.67 \pm 0.00$ & -27.71 & 3.255 & $\ldots$ & $\ldots$ & 180124 \\
\hline 13:00:31.133 & $-28: 29: 31.01$ & $17.94 \pm 0.00$ & -28.18 & 4.710 & $\ldots$ & $\ldots$ & $180124^{\mathrm{d}}$ \\
\hline 13:01:48.270 & $-14: 46: 52.70$ & $18.44 \pm 0.02$ & -27.67 & 4.515 & $\ldots$ & $\cdots$ & 180124 \\
\hline 13:02:30.435 & $-10: 26: 28.59$ & $18.29 \pm 0.01$ & -27.41 & 3.820 & $\ldots$ & $\ldots$ & 180124 \\
\hline 13:05:00.904 & $-12: 26: 18.79$ & $18.17 \pm 0.01$ & -27.09 & 3.080 & $\cdots$ & $\cdots$ & 180405 \\
\hline 13:16:44.039 & $-25: 38: 10.33$ & $18.12 \pm 0.01$ & -27.36 & 3.370 & $\ldots$ & $\cdots$ & 180405 \\
\hline 13:17:25.036 & $-18: 42: 30.76$ & $18.24 \pm 0.01$ & -27.37 & 3.700 & $\cdots$ & $\cdots$ & 180404 \\
\hline 13:29:56.958 & $-04: 52: 21.77$ & $18.23 \pm 0.02$ & -27.26 & 3.395 & $\cdots$ & $\cdots$ & 180406 \\
\hline 13:39:32.277 & $+36: 13: 40.62$ & $18.33 \pm 0.01$ & -27.19 & 3.450 & $\cdots$ & $\ldots$ & 180321 \\
\hline $13: 58: 32.274$ & $-28: 48: 35.48$ & $18.40 \pm 0.02$ & -27.31 & 3.850 & $\cdots$ & $\cdots$ & 180602 \\
\hline 14:00:15.152 & $-03: 44: 16.50$ & $17.60 \pm 0.01$ & -27.95 & 3.540 & $\cdots$ & $\cdots$ & 180404 \\
\hline 14:08:01.817 & $-27: 58: 20.35$ & $17.77 \pm 0.01$ & -28.32 & 4.440 & $\ldots$ & $\cdots$ & 180404 \\
\hline 14:11:42.768 & $-24: 13: 13.48$ & $18.11 \pm 0.01$ & -27.42 & 3.540 & $\ldots$ & $\ldots$ & 180404 \\
\hline $14: 27: 32.247$ & $-18: 03: 18.31$ & $17.07 \pm 0.02$ & -28.22 & 3.115 & $\ldots$ & $\ldots$ & 180404 \\
\hline $14: 32: 54.468$ & $-27: 22: 28.05$ & $18.37 \pm 0.02$ & -27.25 & 3.725 & $\cdots$ & $\cdots$ & 180602 \\
\hline $14: 39: 49.242$ & $-08: 07: 05.38$ & $18.10 \pm 0.01$ & -27.40 & 3.430 & $\ldots$ & $\ldots$ & 180602 \\
\hline $14: 45: 49.741$ & $-11: 10: 15.68$ & $17.94 \pm 0.00$ & -27.28 & 2.995 & $\cdots$ & $\cdots$ & 180404 \\
\hline
\end{tabular}


Table 8

(Continued)

\begin{tabular}{|c|c|c|c|c|c|c|c|}
\hline $\begin{array}{l}\text { R.A. (J2000) } \\
\text { (hh:mm:ss.sss) }\end{array}$ & $\begin{array}{l}\text { Decl. (J2000) } \\
\text { (dd:mm:ss.ss) }\end{array}$ & $\begin{array}{c}m_{i} \\
(\mathrm{mag})\end{array}$ & $\begin{array}{l}M_{1450} \\
(\mathrm{mag})\end{array}$ & $\begin{array}{l}\text { Spectroscopic } \\
\text { Redshift }\end{array}$ & $\begin{array}{l}\text { Near-UV } \\
\text { (mag) }\end{array}$ & $\begin{array}{l}\text { Far-UV } \\
\text { (mag) }\end{array}$ & Notes $^{\mathrm{b}}$ \\
\hline $14: 55: 59.430$ & $-25: 28: 32.10$ & $18.28 \pm 0.01$ & -27.59 & 4.160 & $\ldots$ & $\ldots$ & 180404 \\
\hline 15:18:53.216 & $-11: 59: 51.54$ & $17.83 \pm 0.00$ & -27.68 & 3.435 & $\ldots$ & $\ldots$ & 180404 \\
\hline $15: 23: 12.411$ & $-16: 27: 22.92$ & $17.83 \pm 0.00$ & -28.03 & 4.120 & $\ldots$ & $\ldots$ & 180404 \\
\hline 15:32:45.990 & $-25: 10: 48.22$ & $17.78 \pm 0.01$ & -28.11 & 4.155 & $\ldots$ & $\ldots$ & 180404 \\
\hline $15: 38: 15.568$ & $+81: 44: 32.99$ & $18.06 \pm 0.01$ & -27.27 & 3.180 & $21.43 \pm 0.21$ & $\ldots$ & 180514 \\
\hline $15: 49: 16.840$ & $-22: 37: 46.53$ & $18.06 \pm 0.01$ & -27.68 & 3.905 & $\ldots$ & $\ldots$ & 180405 \\
\hline $15: 56: 35.483$ & $+60: 37: 26.78$ & $17.69 \pm 0.00$ & -27.77 & 3.340 & $\ldots$ & $\ldots$ & 180517 \\
\hline $15: 58: 41.854$ & $-04: 03: 53.42$ & $17.68 \pm 0.01$ & -27.55 & 3.020 & $\ldots$ & $\ldots$ & 180405 \\
\hline $16: 00: 19.482$ & $-12: 17: 02.75$ & $18.07 \pm 0.01$ & -27.14 & 2.980 & $\ldots$ & $\ldots$ & 180405 \\
\hline 16:01:11.971 & $-16: 43: 41.63$ & $18.12 \pm 0.01$ & -26.99 & 2.840 & $\ldots$ & $\ldots$ & 180602 \\
\hline 16:06:08.999 & $+48: 41: 37.02$ & $18.04 \pm 0.01$ & -27.42 & 3.345 & $21.39 \pm 0.15$ & $\ldots$ & 180517 \\
\hline $16: 16: 48.948$ & $-09: 14: 44.39$ & $17.39 \pm 0.01$ & -28.45 & 4.055 & $\ldots$ & $\ldots$ & 180405 \\
\hline $16: 17: 37.785$ & $+59: 50: 20.13$ & $17.39 \pm 0.02$ & -28.62 & 4.315 & $\ldots$ & $\ldots$ & 180517 \\
\hline $16: 23: 49.985$ & $-15: 44: 27.68$ & $17.81 \pm 0.03$ & -27.35 & 2.900 & $\ldots$ & $\ldots$ & 180406 \\
\hline $16: 29: 36.489$ & $+60: 40: 49.19$ & $17.93 \pm 0.01$ & -27.24 & 2.940 & $\ldots$ & $\ldots$ & 180517 \\
\hline $16: 31: 18.216$ & $-12: 43: 08.60$ & $16.96 \pm 0.01$ & -28.57 & 3.455 & $\ldots$ & $\ldots$ & 180406 \\
\hline $16: 32: 27.929$ & $-14: 20: 44.18$ & $17.00 \pm 0.01$ & -28.68 & 3.755 & $\ldots$ & $\ldots$ & 180406 \\
\hline $16: 35: 36.073$ & $+03: 24: 07.80$ & $16.95 \pm 0.00$ & -28.50 & 3.320 & $20.54 \pm 0.15$ & $21.53 \pm 0.35$ & 180321 \\
\hline $16: 38: 56.009$ & $+69: 18: 15.24$ & $18.02 \pm 0.01$ & -27.48 & 3.415 & $\ldots$ & $\ldots$ & 180517 \\
\hline $16: 39: 26.455$ & $+03: 52: 04.12$ & $17.52 \pm 0.00$ & -27.86 & 3.245 & $\ldots$ & $\ldots$ & 180321 \\
\hline $16: 48: 52.744$ & $+09: 59: 42.10$ & $17.77 \pm 0.00$ & -27.39 & 2.905 & $20.80 \pm 0.09$ & $21.28 \pm 0.12$ & 180321 \\
\hline 16:59:29.379 & $+65: 38: 20.85$ & $18.35 \pm 0.00$ & -27.33 & 3.805 & $\ldots$ & $\ldots$ & 180514 \\
\hline $17: 20: 46.132$ & $+00: 43: 28.19$ & $17.91 \pm 0.01$ & -27.57 & 3.375 & $\ldots$ & $\ldots$ & 180320 \\
\hline 17:30:03.673 & $+48: 46: 30.77$ & $16.97 \pm 0.01$ & -28.51 & 3.355 & $\ldots$ & $\ldots$ & 180518 \\
\hline $17: 47: 13.484$ & $+29: 55: 32.30$ & $18.34 \pm 0.01$ & -27.17 & 3.465 & $\ldots$ & $\ldots$ & 180321 \\
\hline $17: 53: 34.530$ & $+37: 49: 07.13$ & $18.19 \pm 0.01$ & -27.00 & 2.945 & $\ldots$ & $\ldots$ & 180518 \\
\hline $17: 55: 21.128$ & $+30: 09: 04.24$ & $17.89 \pm 0.00$ & -27.34 & 3.025 & $\ldots$ & $\ldots$ & 180321 \\
\hline $17: 56: 29.853$ & $+26: 07: 40.57$ & $17.27 \pm 0.01$ & -28.26 & 3.480 & $\ldots$ & $\ldots$ & 180320 \\
\hline 18:02:09.690 & $+40: 12: 53.77$ & $18.16 \pm 0.01$ & -27.37 & 3.525 & $\ldots$ & $\ldots$ & 180518 \\
\hline 18:10:27.309 & $+34: 24: 08.85$ & $18.26 \pm 0.01$ & -27.19 & 3.330 & $\ldots$ & $\ldots$ & 171109 \\
\hline $18: 10: 41.346$ & $+34: 54: 49.46$ & $18.04 \pm 0.01$ & -27.47 & 3.460 & $\ldots$ & $\ldots$ & 171109 \\
\hline $18: 17: 06.185$ & $+48: 22: 26.07$ & $17.39 \pm 0.02$ & -28.25 & 3.705 & $\ldots$ & $\ldots$ & 180518 \\
\hline $18: 19: 14.803$ & $+33: 39: 45.98$ & $17.74 \pm 0.00$ & -27.84 & 3.610 & $\ldots$ & $\ldots$ & 171020 \\
\hline $19: 35: 12.403$ & $-26: 10: 49.63$ & $17.20 \pm 0.00$ & -28.13 & 3.175 & $\ldots$ & $\ldots$ & 171007 \\
\hline 20:00:13.515 & $-25: 05: 36.92$ & $17.72 \pm 0.01$ & -27.84 & 3.575 & $\ldots$ & $\cdots$ & 171006 \\
\hline 20:02:05.969 & $-23: 28: 26.52$ & $17.82 \pm 0.01$ & -27.36 & 2.940 & $\ldots$ & $\ldots$ & 171007 \\
\hline $20: 10: 23.353$ & $-18: 23: 47.75$ & $18.15 \pm 0.00$ & -27.36 & 3.440 & $\ldots$ & $\ldots$ & 171010 \\
\hline $20: 11: 58.767$ & $-26: 23: 40.95$ & $17.47 \pm 0.00$ & -28.12 & 3.620 & $\ldots$ & $\ldots$ & 171008 \\
\hline $20: 17: 41.494$ & $-28: 16: 29.96$ & $17.40 \pm 0.01$ & -28.22 & 3.690 & $\ldots$ & $\ldots$ & 171008 \\
\hline $20: 18: 34.860$ & $-15: 28: 38.69$ & $17.35 \pm 0.01$ & -28.22 & 3.580 & $\ldots$ & $\ldots$ & 171007 \\
\hline $20: 20: 43.904$ & $-02: 37: 02.52$ & $17.75 \pm 0.01$ & -27.81 & 3.585 & $\ldots$ & $\ldots$ & 171008 \\
\hline $20: 33: 36.699$ & $-24: 56: 15.86$ & $18.14 \pm 0.01$ & -27.38 & 3.470 & $\ldots$ & $\ldots$ & 171010 \\
\hline $20: 33: 43.573$ & $-30: 23: 09.83$ & $17.76 \pm 0.00$ & -27.77 & 3.520 & $\ldots$ & $\ldots$ & 171007 \\
\hline $20: 34: 16.997$ & $-02: 59: 53.43$ & $18.26 \pm 0.00$ & -27.01 & 3.090 & $\ldots$ & $\ldots$ & 180602 \\
\hline 20:36:23.526 & $-08: 37: 29.92$ & $17.51 \pm 0.01$ & -27.63 & 2.895 & $\ldots$ & $\ldots$ & 171008 \\
\hline $20: 43: 20.175$ & $-03: 38: 40.93$ & $17.48 \pm 0.02$ & -27.69 & 2.925 & $\cdots$ & $\ldots$ & 171009 \\
\hline $20: 48: 48.274$ & $-22: 51: 52.15$ & $17.18 \pm 0.00$ & -28.10 & 3.100 & $\ldots$ & $\ldots$ & 171009 \\
\hline 21:03:51.499 & $-26: 23: 00.22$ & $17.54 \pm 0.00$ & -27.98 & 3.455 & $\ldots$ & $\ldots$ & 171006 \\
\hline 21:11:11.604 & $-25: 36: 15.03$ & $17.96 \pm 0.01$ & -27.52 & 3.370 & $\ldots$ & $\ldots$ & 171007 \\
\hline $21: 25: 40.966$ & $-17: 19: 51.41$ & $16.42 \pm 0.00$ & -29.35 & 3.900 & $\ldots$ & $\ldots$ & 171010 \\
\hline $21: 26: 51.969$ & $-10: 31: 39.62$ & $18.21 \pm 0.01$ & -27.51 & 3.870 & $\ldots$ & $\ldots$ & 171008 \\
\hline $21: 27: 16.485$ & $-04: 04: 33.58$ & $18.47 \pm 0.02$ & -27.05 & 3.545 & $\ldots$ & $\ldots$ & 171010 \\
\hline 21:30:50.101 & $-24: 44: 03.50$ & $18.01 \pm 0.01$ & -27.48 & 3.380 & $\cdots$ & $\ldots$ & 171009 \\
\hline $21: 32: 25.900$ & $-28: 31: 33.33$ & $17.76 \pm 0.01$ & -27.32 & 2.810 & $\ldots$ & $\ldots$ & 171009 \\
\hline $21: 34: 45.240$ & $-27: 49: 39.75$ & $17.68 \pm 0.00$ & -27.58 & 3.065 & $22.89 \pm 0.47$ & $\ldots$ & 171009 \\
\hline 22:21:52.882 & $-18: 26: 02.94$ & $17.80 \pm 0.01$ & -28.30 & 4.520 & $\ldots$ & $\ldots$ & 171010 \\
\hline 22:29:59.998 & $-26: 21: 05.68$ & $17.91 \pm 0.01$ & -27.38 & 3.140 & $\ldots$ & $\ldots$ & 171008 \\
\hline $22: 30: 49.477$ & $-21: 54: 02.09$ & $18.30 \pm 0.01$ & -27.26 & 3.605 & $\ldots$ & $\ldots$ & 180602 \\
\hline $22: 37: 34.408$ & $-31: 07: 14.09$ & $17.87 \pm 0.01$ & -27.42 & 3.120 & $\ldots$ & $\ldots$ & 171009 \\
\hline $22: 46: 19.167$ & $-25: 17: 20.76$ & $17.76 \pm 0.01$ & -27.40 & 2.900 & $21.71 \pm 0.27$ & $22.55 \pm 0.54$ & 171007 \\
\hline 22:56:33.174 & $-12: 43: 59.64$ & $17.62 \pm 0.01$ & -27.48 & 2.825 & $\ldots$ & $\ldots$ & 171009 \\
\hline $22: 58: 20.943$ & $-28: 18: 55.25$ & $18.07 \pm 0.01$ & -27.46 & 3.525 & $\ldots$ & $\ldots$ & 171009 \\
\hline $22: 59: 39.043$ & $-22: 50: 35.10$ & $17.43 \pm 0.01$ & -28.08 & 3.470 & $\ldots$ & $\ldots$ & 171007 \\
\hline
\end{tabular}


Table 8

(Continued)

\begin{tabular}{|c|c|c|c|c|c|c|c|}
\hline $\begin{array}{l}\text { R.A. (J2000) } \\
\text { (hh:mm:ss.sss) }\end{array}$ & $\begin{array}{l}\text { Decl. (J2000) } \\
\text { (dd:mm:ss.ss) }\end{array}$ & $\begin{array}{c}m_{i} \\
(\mathrm{mag})\end{array}$ & $\begin{array}{l}M_{1450} \\
\text { (mag) }\end{array}$ & $\begin{array}{l}\text { Spectroscopic } \\
\text { Redshift }\end{array}$ & $\begin{array}{l}\text { Near-UV } \\
\quad(\mathrm{mag})\end{array}$ & $\begin{array}{l}\text { Far-UV } \\
\text { (mag) }\end{array}$ & Notes $^{b}$ \\
\hline $23: 00: 22.023$ & $-14: 40: 31.37$ & $18.28 \pm 0.00$ & -27.43 & 3.850 & $\cdots$ & $\cdots$ & 171008 \\
\hline 23:04:32.312 & $-12: 48: 19.64$ & $18.13 \pm 0.01$ & -27.58 & 3.850 & $\ldots$ & $\cdots$ & 171007 \\
\hline 23:08:27.042 & $-13: 32: 56.21$ & $17.71 \pm 0.01$ & -28.00 & 3.830 & $\cdots$ & $\cdots$ & 171006 \\
\hline 23:09:59.293 & $-12: 26: 03.16$ & $17.94 \pm 0.00$ & -27.70 & 3.730 & $\cdots$ & $\cdots$ & 171006 \\
\hline 23:23:06.892 & $-24: 51: 13.78$ & $18.35 \pm 0.01$ & -27.36 & 3.860 & $\cdots$ & $\cdots$ & 171007 \\
\hline $23: 39: 26.346$ & $-11: 20: 50.35$ & $18.20 \pm 0.01$ & -27.64 & 4.090 & $\cdots$ & $\cdots$ & 171021 \\
\hline $23: 42: 10.117$ & $-23: 21: 53.60$ & $18.09 \pm 0.01$ & -27.43 & 3.480 & $\cdots$ & $\cdots$ & 171008 \\
\hline $23: 42: 41.975$ & $-12: 26: 55.02$ & $17.98 \pm 0.00$ & -27.51 & 3.380 & $\cdots$ & $\cdots$ & 171006 \\
\hline
\end{tabular}

Notes.

a The near and far-UV magnitudes were obtained from cross-matches within 2".0 to the GALEX GR6/7 data release.

$\mathrm{b}$ This column shows the observation date (YYMMDD) and provides further information on individual objects.

${ }^{c}$ This object has been classified as a BAL, LoBAL, or FeLoBAL quasar. Details are discussed in Section 7.2.

${ }^{d}$ These objects were also independently discovered by Yang et al. (2019b).

(This table is available in machine-readable form.) 


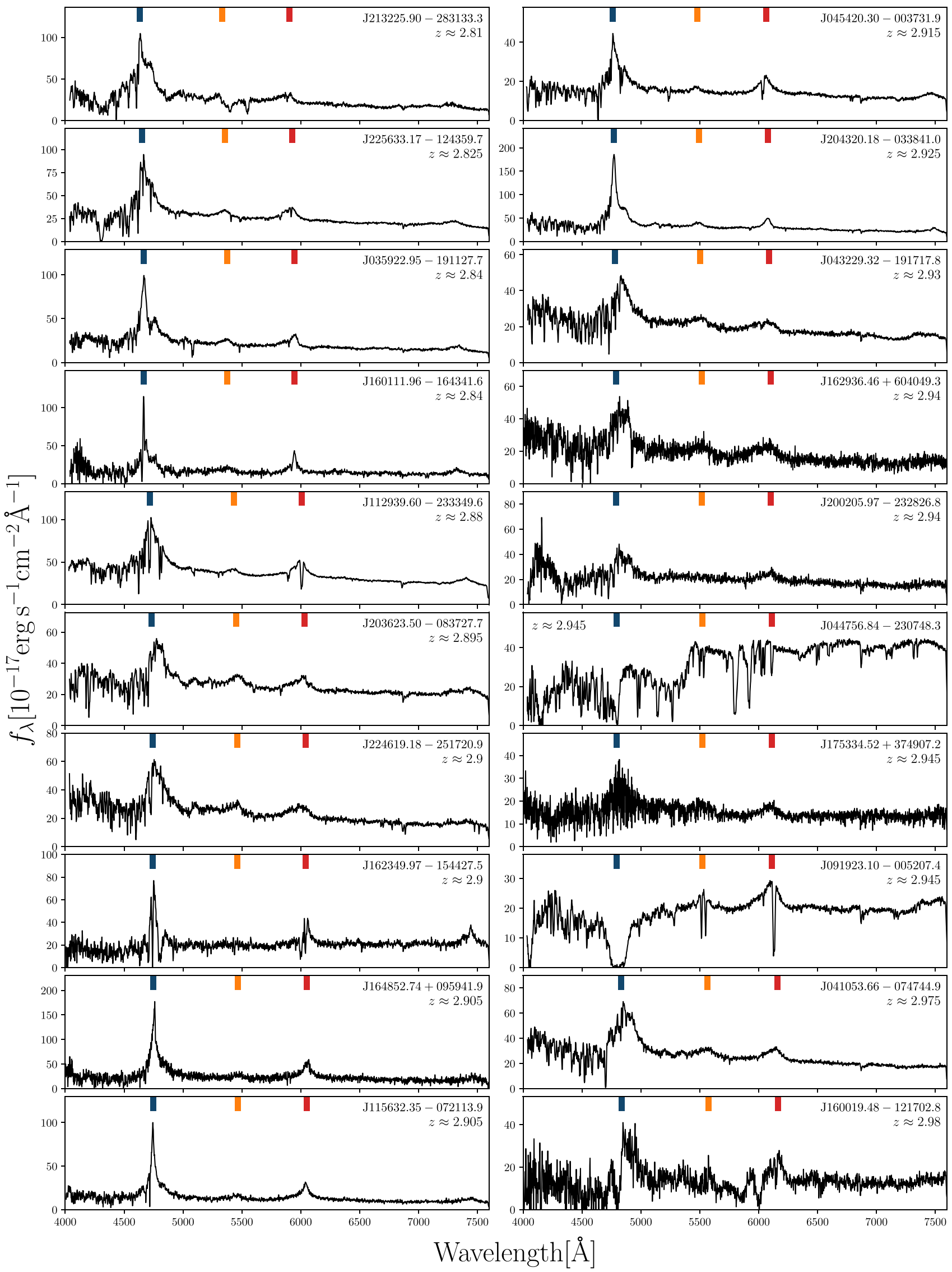

Figure 9. Discovery spectra of the newly discovered PS-ELQS quasars. The dark blue, orange, and red bars denote the center positions of the broad Ly $\alpha$, Si IV, and C IV emission lines according to the spectroscopic redshift. 


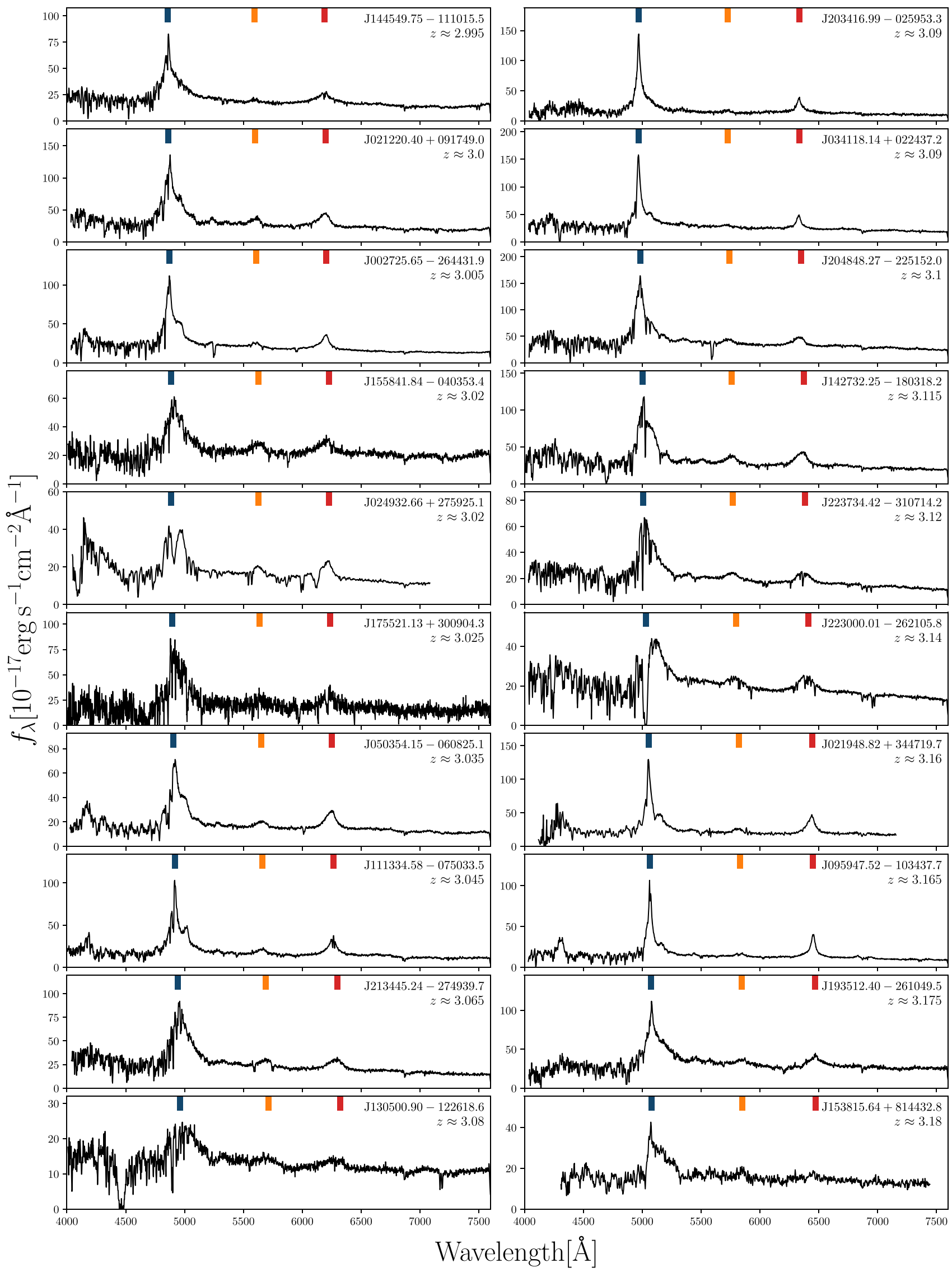

Figure 9. (Continued.) 


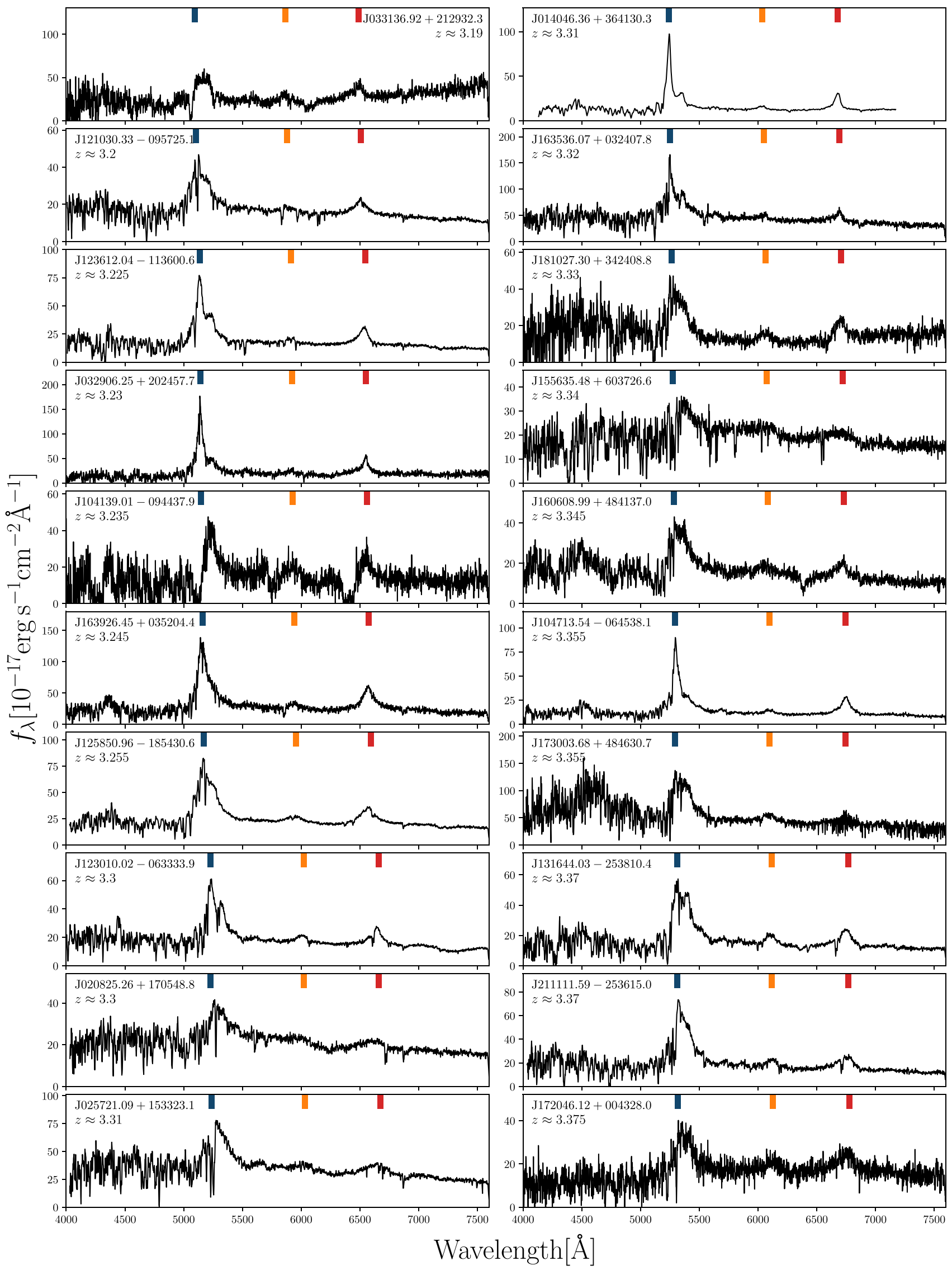

Figure 9. (Continued.) 


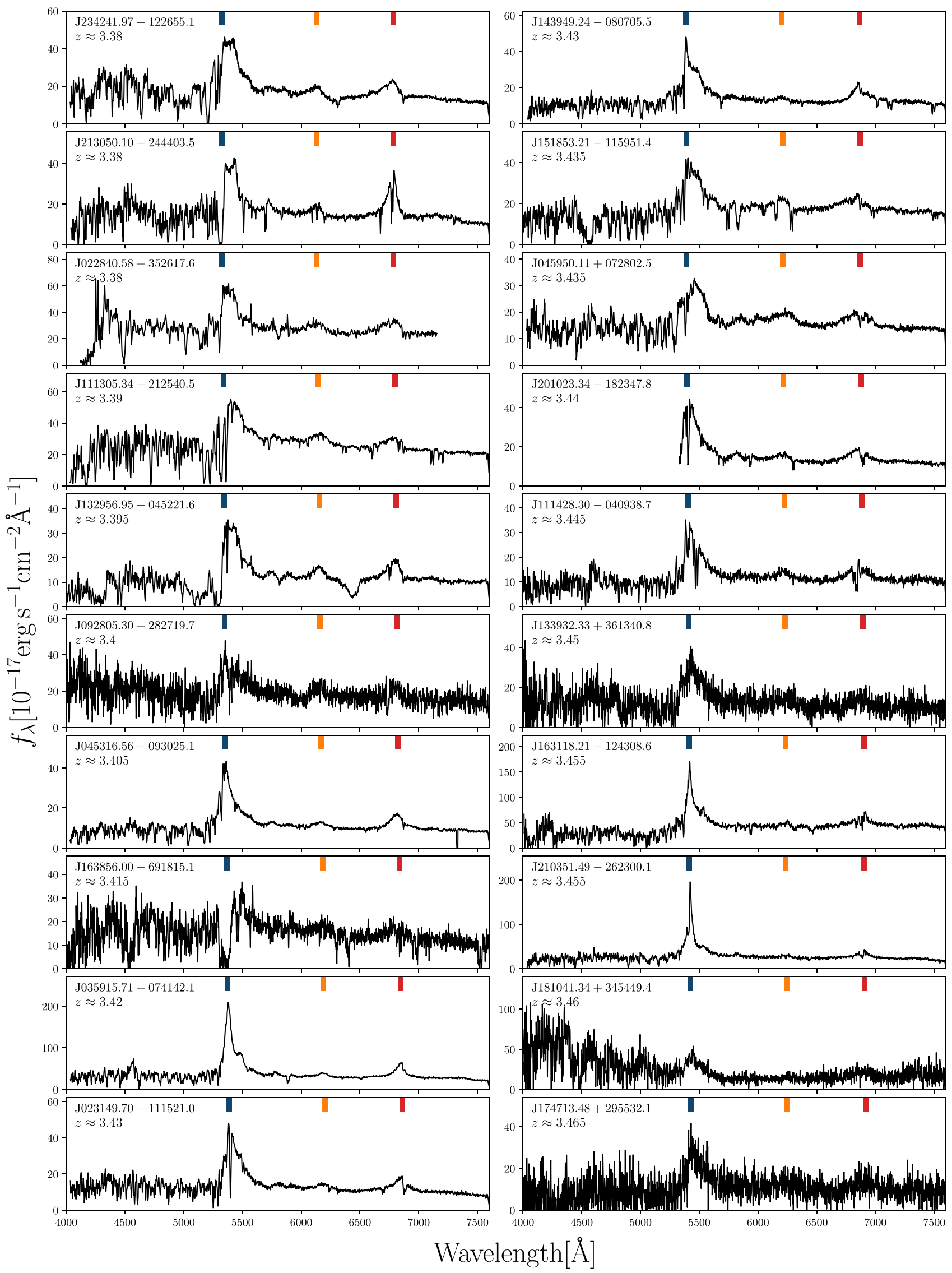

Figure 10. Discovery spectra of the newly discovered PS-ELQS quasars (continued). 


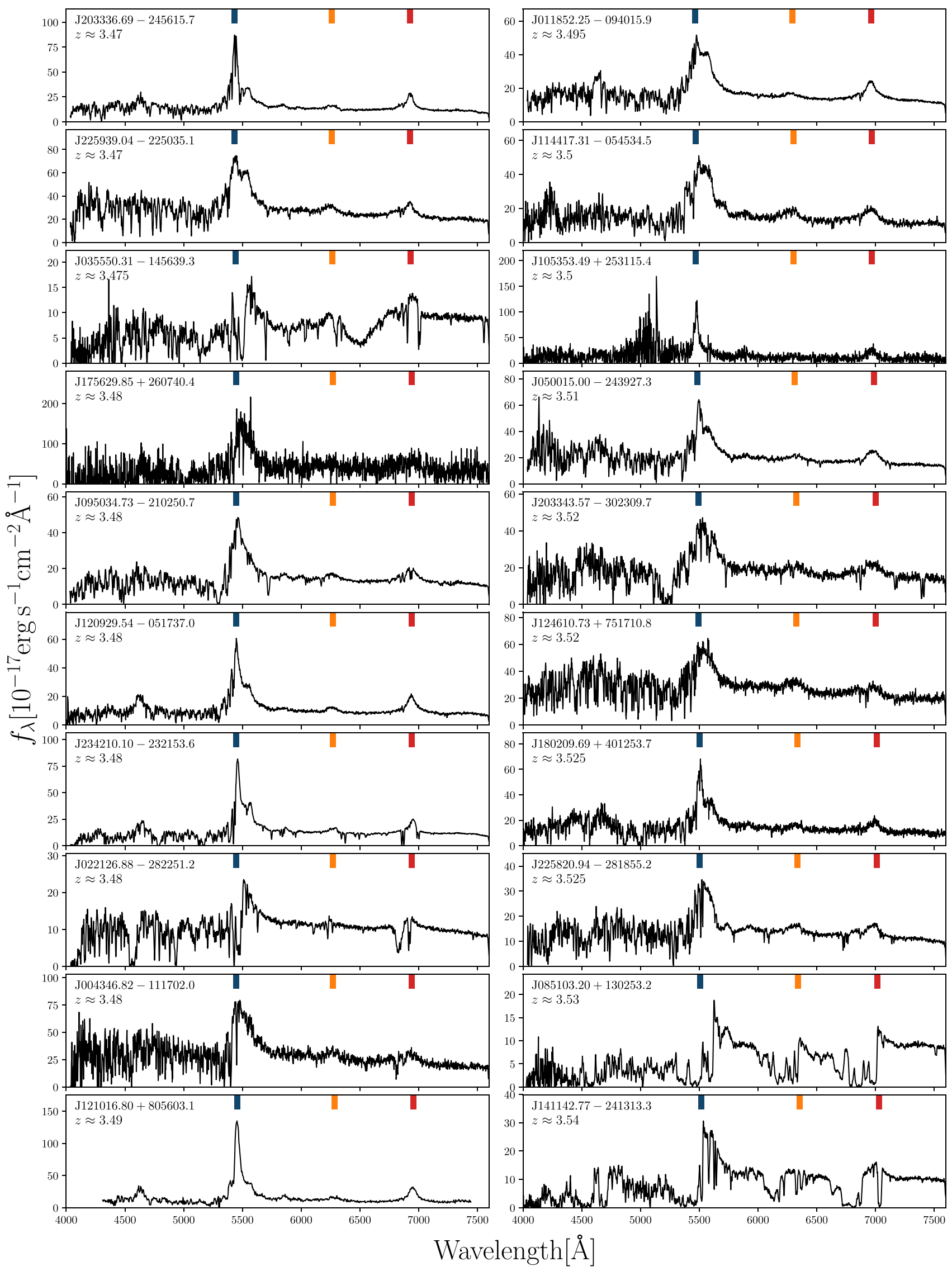

Figure 10. (Continued.) 


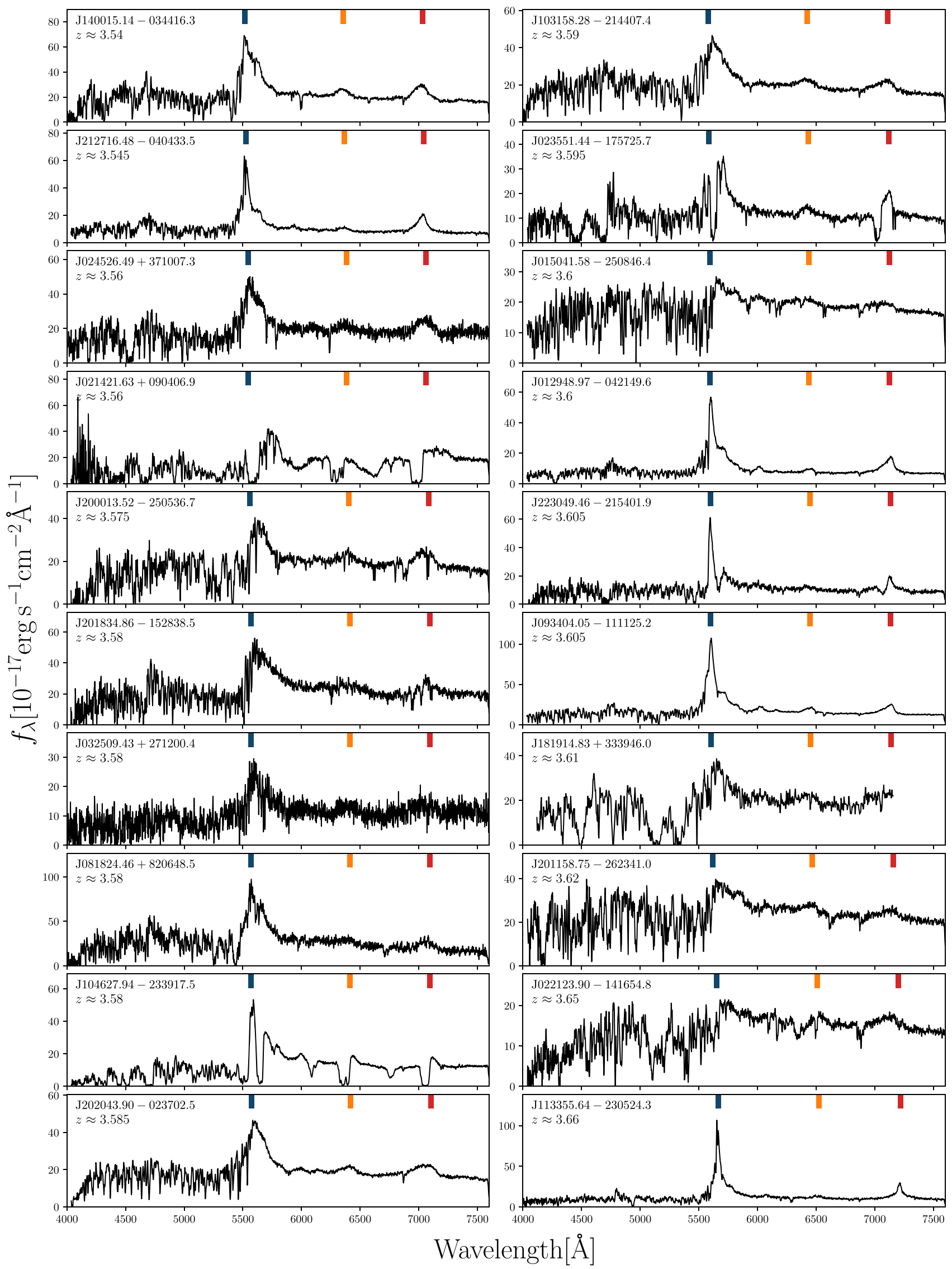

Figure 10. (Continued.) 


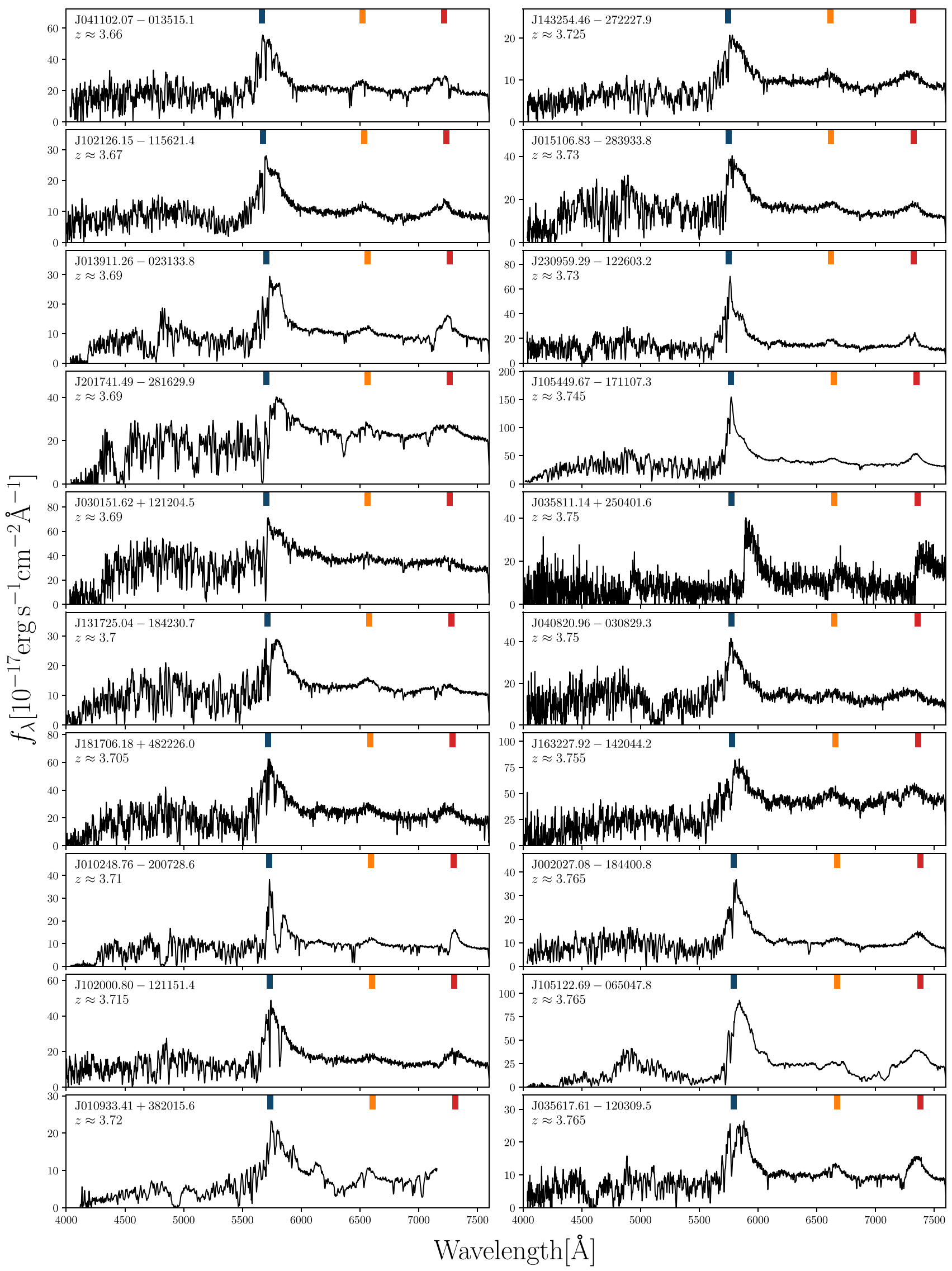

Figure 11. Discovery spectra of the newly discovered PS-ELQS quasars (continued). 


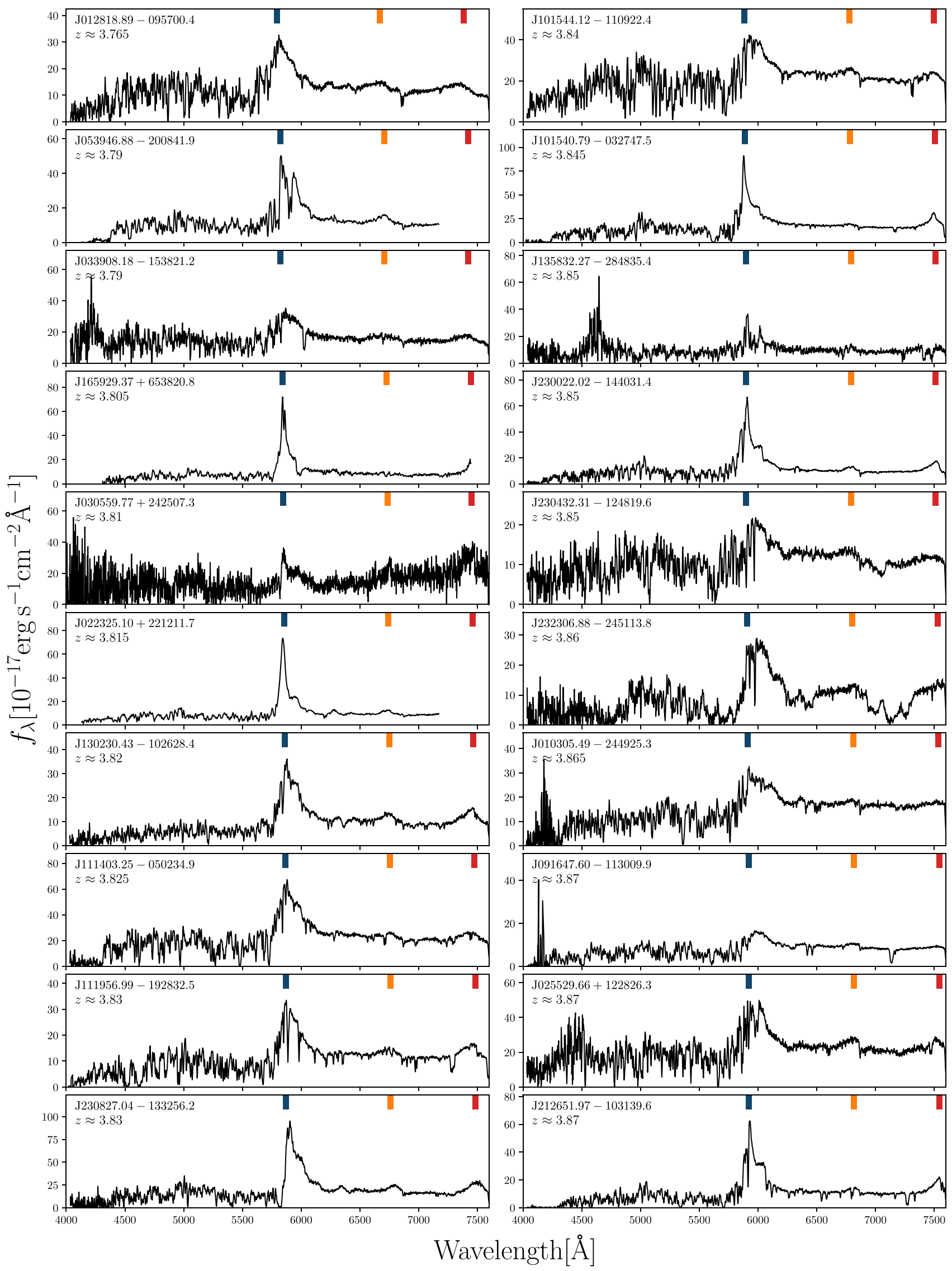

Figure 11. (Continued.) 


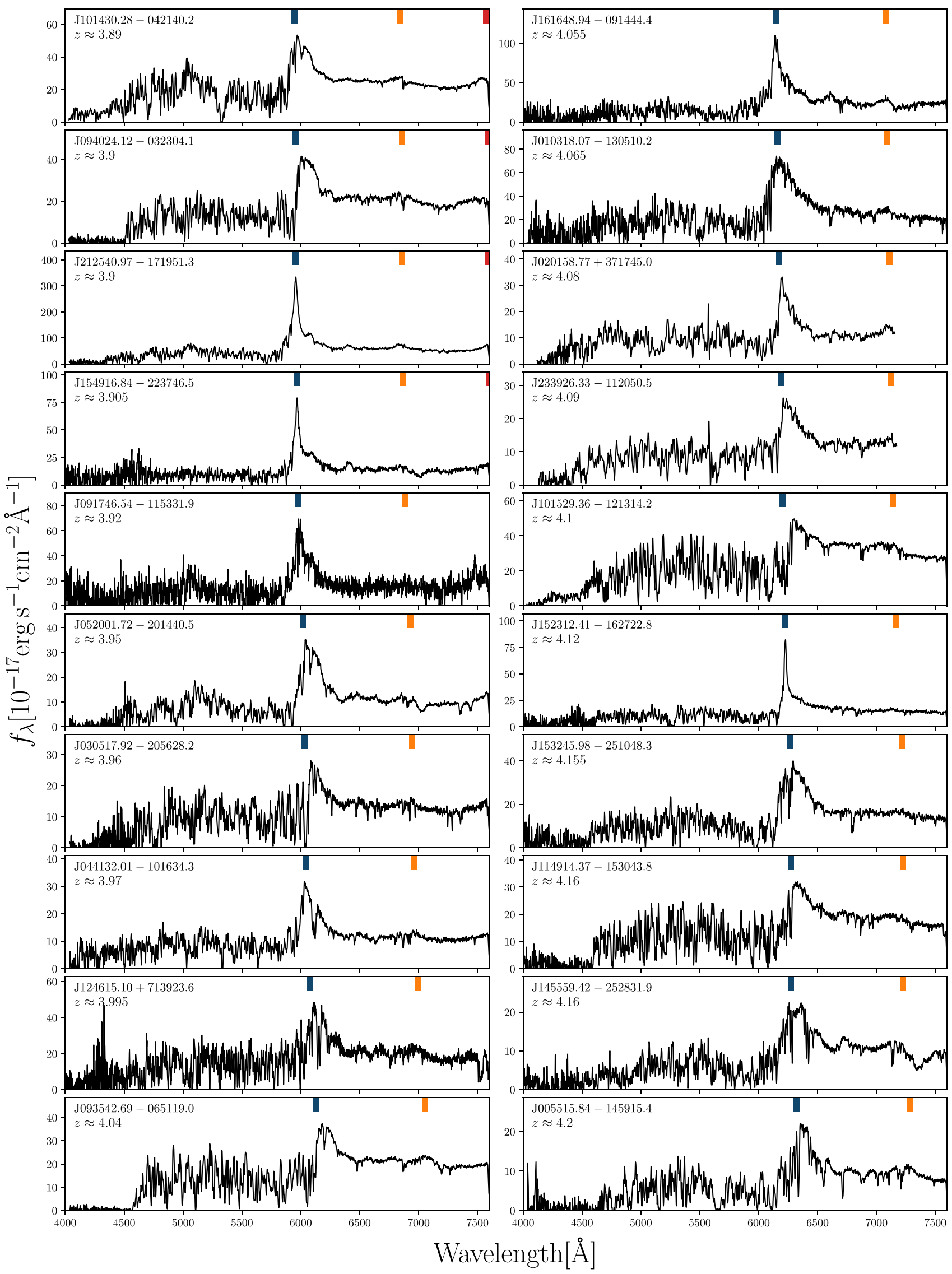

Figure 11. (Continued.) 


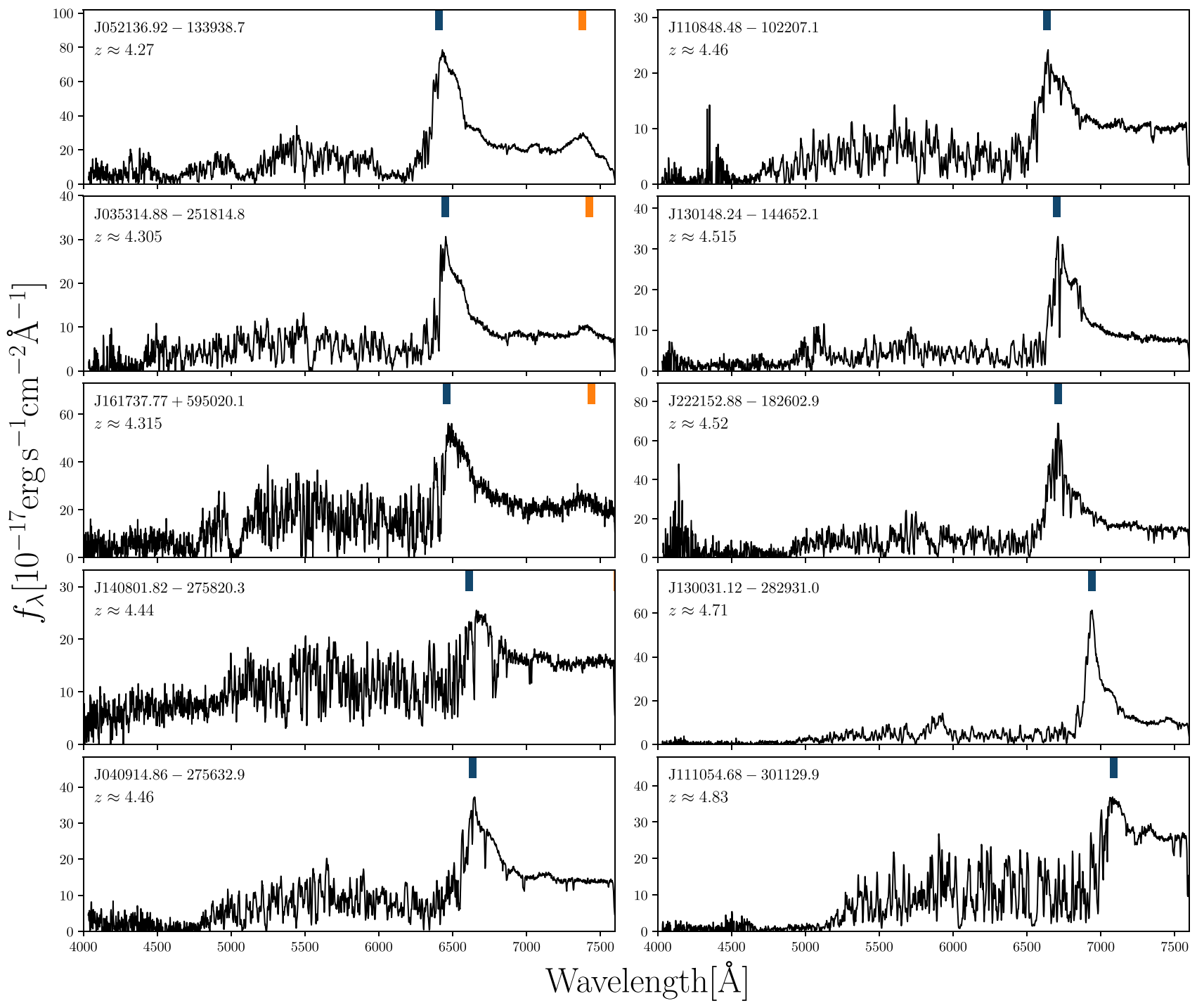

Figure 11. (Continued.) 
Appendix C

Newly Discovered QSOs at Lower Redshift $(z<2.8)$

In this part of the Appendix we present newly discovered quasars targeted with PS-ELQS spectroscopically confirmed to be at $z<2.8$. A summary of the general properties of these objects in provided in Table 9, which is also available in machine-readable format online. The discovery spectra are shown in Figure 12.

Table 9

Newly Discovered Quasars at $z<2.8$ in the PS-ELQS Sample

\begin{tabular}{|c|c|c|c|c|c|c|c|}
\hline $\begin{array}{l}\text { R.A. (J2000) } \\
\text { (hh:mm:ss.sss) }\end{array}$ & $\begin{array}{l}\text { Decl. (J2000) } \\
\text { (dd:mm:ss.ss) }\end{array}$ & $\begin{array}{c}m_{i} \\
(\mathrm{mag})\end{array}$ & $\begin{array}{l}M_{1450} \\
(\mathrm{mag})\end{array}$ & $\begin{array}{l}\text { Spectroscopic } \\
\text { Redshift }\end{array}$ & $\begin{array}{l}\text { Near-UV } \\
(\mathrm{mag})\end{array}$ & $\begin{array}{l}\text { Far-UV } \\
(\mathrm{mag})\end{array}$ & Notes $^{\mathrm{b}}$ \\
\hline $00: 13: 10.727$ & $+29: 18: 47.74$ & $17.65 \pm 0.01$ & -27.27 & 2.500 & $\ldots$ & $\ldots$ & 171020 \\
\hline 00:38:56.987 & $-29: 22: 24.43$ & $17.35 \pm 0.00$ & -27.28 & 2.270 & $\ldots$ & $\ldots$ & $180124^{\mathrm{c}}$ \\
\hline 02:11:19.800 & $-19: 59: 43.01$ & $17.94 \pm 0.01$ & -26.93 & 2.450 & $\ldots$ & $\cdots$ & $171010^{\mathrm{c}}$ \\
\hline 02:35:00.447 & $+02: 38: 29.25$ & $18.11 \pm 0.01$ & -23.73 & 0.650 & $\cdots$ & $\cdots$ & $180124^{\mathrm{c}}$ \\
\hline 03:30:11.020 & $-12: 40: 08.68$ & $17.59 \pm 0.00$ & -26.78 & 2.075 & $\ldots$ & $\ldots$ & 171006 \\
\hline 03:35:59.996 & $-13: 26: 02.08$ & $17.99 \pm 0.01$ & -26.14 & 1.900 & $\ldots$ & $\ldots$ & $171007^{\mathrm{c}}$ \\
\hline 03:41:38.070 & $-11: 42: 59.44$ & $17.09 \pm 0.01$ & -27.98 & 2.770 & $\ldots$ & $\ldots$ & 171008 \\
\hline 04:05:48.525 & $-24: 21: 15.26$ & $17.38 \pm 0.01$ & -27.68 & 2.760 & $\ldots$ & $\ldots$ & 171007 \\
\hline 09:10:54.661 & $+46: 06: 51.94$ & $18.09 \pm 0.01$ & -26.81 & 2.490 & $\ldots$ & $\ldots$ & 180514 \\
\hline 09:26:42.056 & $-17: 47: 21.96$ & $18.24 \pm 0.02$ & -22.39 & 0.369 & $20.10 \pm 0.10$ & $20.52 \pm 0.17$ & 180602 \\
\hline 09:36:20.407 & $+82: 51: 14.07$ & $17.54 \pm 0.01$ & -27.50 & 2.715 & $\ldots$ & $\ldots$ & 180517 \\
\hline 10:11:22.657 & $-24: 33: 01.43$ & $18.12 \pm 0.01$ & -26.92 & 2.720 & $\ldots$ & $\ldots$ & 180123 \\
\hline 10:57:02.777 & $+34: 22: 50.37$ & $18.08 \pm 0.00$ & -26.63 & 2.320 & $\ldots$ & $\ldots$ & 180321 \\
\hline $11: 28: 14.210$ & $+26: 56: 46.36$ & $18.18 \pm 0.01$ & -26.32 & 2.170 & $\ldots$ & $\ldots$ & 180321 \\
\hline $11: 32: 52.869$ & $-06: 32: 43.31$ & $17.68 \pm 0.01$ & -27.15 & 2.410 & $\ldots$ & $\ldots$ & $180404^{\mathrm{c}}$ \\
\hline $14: 27: 45.083$ & $-14: 51: 49.32$ & $17.79 \pm 0.01$ & -26.96 & 2.350 & $\ldots$ & $\ldots$ & 180404 \\
\hline $14: 40: 30.602$ & $+69: 42: 11.58$ & $17.99 \pm 0.01$ & -27.04 & 2.690 & $\ldots$ & $\ldots$ & 180514 \\
\hline 17:13:01.101 & $+66: 58: 25.90$ & $18.29 \pm 0.01$ & -26.67 & 2.550 & $\ldots$ & $\ldots$ & 180518 \\
\hline $18: 00: 30.260$ & $+79: 34: 47.07$ & $17.93 \pm 0.01$ & -27.12 & 2.755 & $22.06 \pm 0.30$ & $\ldots$ & 180518 \\
\hline 18:03:11.956 & $+70: 38: 25.75$ & $17.94 \pm 0.01$ & -27.09 & 2.715 & $\ldots$ & $\ldots$ & 180518 \\
\hline $18: 07: 24.633$ & $+28: 08: 14.40$ & $17.90 \pm 0.00$ & -26.60 & 2.150 & $\ldots$ & $\ldots$ & 171020 \\
\hline $18: 20: 00.261$ & $+63: 10: 36.85$ & $17.63 \pm 0.00$ & -26.97 & 2.235 & $\cdots$ & $\cdots$ & 180518 \\
\hline 18:29:04.759 & $+78: 31: 06.45$ & $18.13 \pm 0.01$ & -26.41 & 2.200 & $22.33 \pm 0.35$ & $\ldots$ & 180514 \\
\hline 19:19:46.075 & $+74: 37: 47.11$ & $17.86 \pm 0.01$ & -25.92 & 1.604 & $\ldots$ & $\ldots$ & $180518^{c}$ \\
\hline 20:30:34.859 & $-25: 41: 57.41$ & $18.10 \pm 0.01$ & -26.96 & 2.743 & $\ldots$ & $\ldots$ & 180604 \\
\hline 22:09:12.009 & $+06: 19: 20.01$ & $17.25 \pm 0.00$ & -26.90 & 1.910 & $\ldots$ & $\ldots$ & $171008^{c}$ \\
\hline 22:51:59.483 & $+17: 28: 44.68$ & $17.35 \pm 0.01$ & -27.36 & 2.320 & $\ldots$ & $\ldots$ & 180518 \\
\hline 23:41:20.021 & $+31: 20: 25.38$ & $18.39 \pm 0.01$ & -26.22 & 2.240 & $\ldots$ & $\ldots$ & 171021 \\
\hline
\end{tabular}

Notes.

a The near and far-UV magnitudes were obtained from cross-matches within 2!"0 to the GALEX GR6/7 data release.

$\mathrm{b}$ This column shows the observation date (YYMMDD) and provides further information on individual objects.

${ }^{c}$ This object has been classified as a BAL, LoBAL, or FeLoBAL quasar. Details are discussed in Section 7.2.

(This table is available in machine-readable form.) 


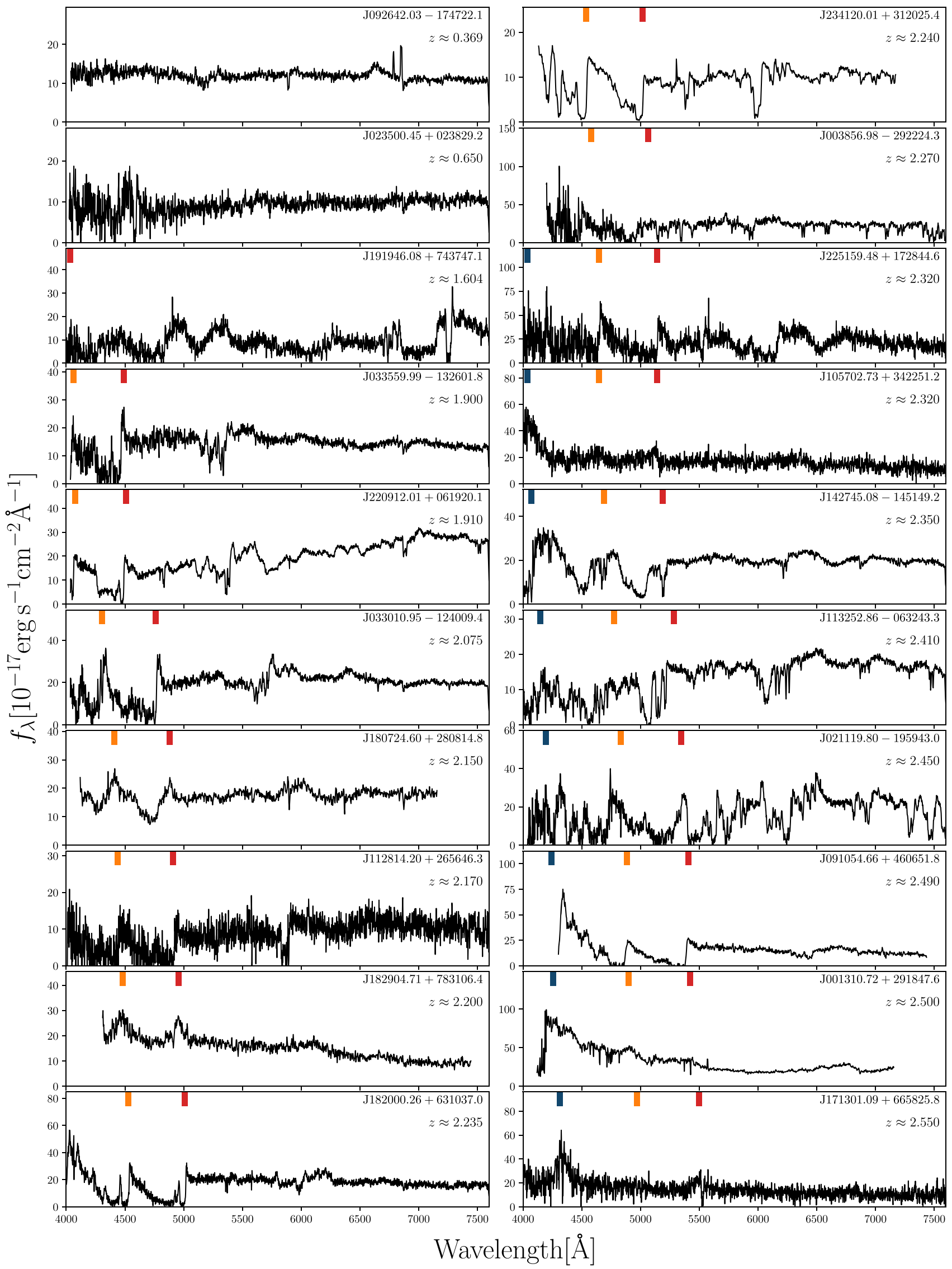

Figure 12. Discovery spectra of the newly discovered PS-ELQS quasars at $z<2.8$. The dark blue, orange, and red bars denote the center positions of the broad Ly $\alpha$, Si IV, and C IV emission lines according to the spectroscopic redshift. 


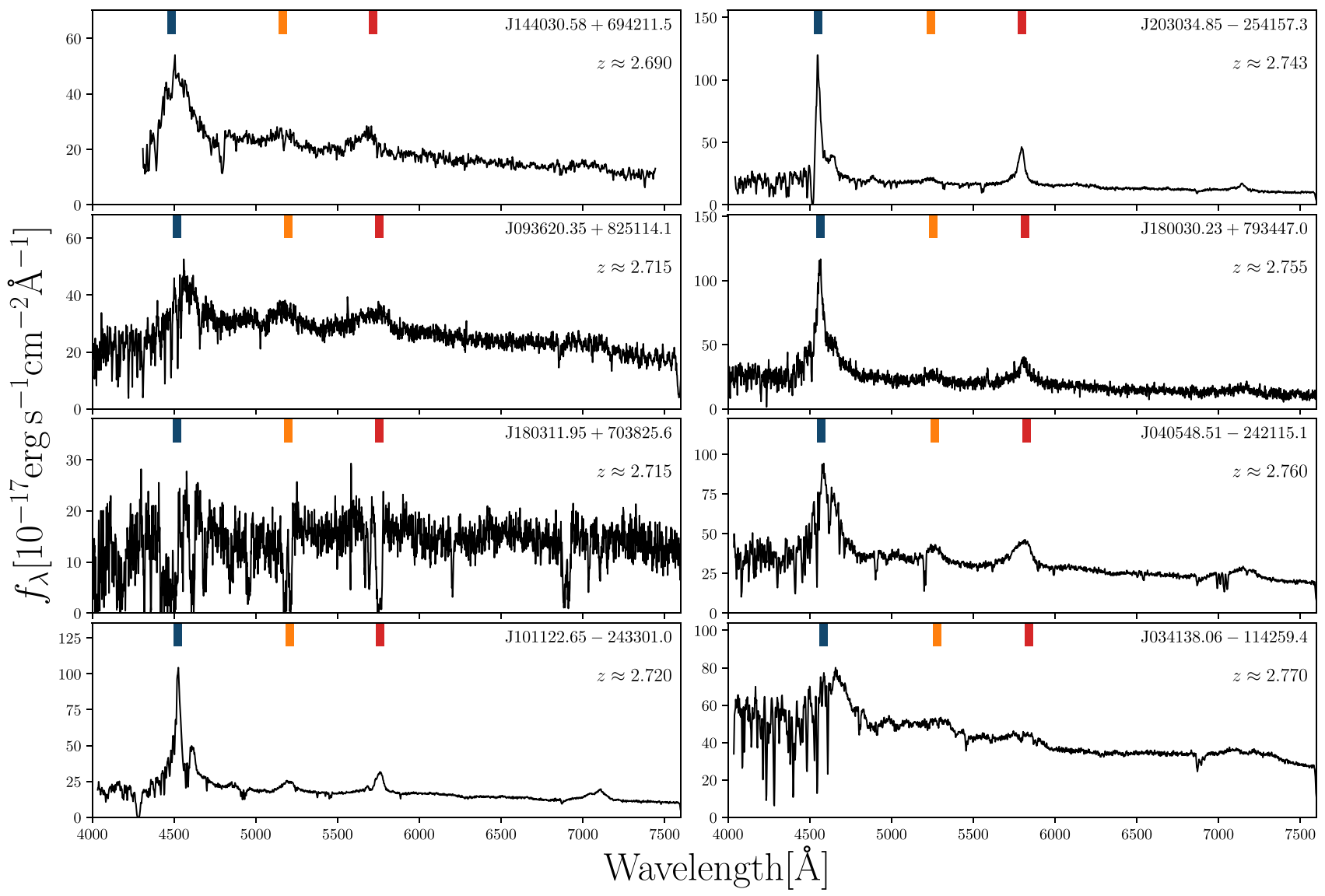

Figure 12. (Continued.) 


\section{Appendix D}

\section{Remaining Good PS-ELQS Candidates}

We further present properties of the remaining good PSELQS candidates in Table 10. A machine-readable version of this table is provided online.

Table 10

Properties of the Remaining Good PS-ELQS Candidates

\begin{tabular}{|c|c|c|c|c|c|c|}
\hline $\begin{array}{l}\text { R.A. (J2000) } \\
\text { (hh:mm:ss.sss) }\end{array}$ & $\begin{array}{l}\text { Decl. (J2000) } \\
\text { (dd:mm:ss.ss) }\end{array}$ & $\begin{array}{c}m_{i} \\
(\mathrm{mag})\end{array}$ & rf_photoz & rf_qso_prob & rf_mult_class_pred & Priority \\
\hline 00:08:01.926 & $-27: 24: 29.28$ & $18.04 \pm 0.01$ & 2.83 & 1.00 & midz & 5 \\
\hline $00: 25: 18.462$ & $-15: 57: 50.47$ & $18.12 \pm 0.01$ & 2.82 & 0.99 & $\operatorname{midz}$ & 5 \\
\hline $00: 46: 12.529$ & $+41: 50: 02.49$ & $18.34 \pm 0.01$ & 3.30 & 0.84 & highz & 4 \\
\hline 00:55:09.859 & $-03: 49: 43.89$ & $18.32 \pm 0.01$ & 2.85 & 0.86 & $\operatorname{midz}$ & 5 \\
\hline 01:54:15.903 & $+40: 43: 40.91$ & $18.02 \pm 0.01$ & 3.59 & 0.86 & highz & 3 \\
\hline 01:58:16.432 & $-01: 30: 38.31$ & $18.18 \pm 0.01$ & 2.88 & 0.98 & $\operatorname{midz}$ & 5 \\
\hline 02:22:07.117 & $-16: 28: 11.77$ & $18.15 \pm 0.01$ & 2.89 & 0.99 & $\operatorname{midz}$ & 5 \\
\hline 02:29:43.899 & $+29: 33: 05.50$ & $18.34 \pm 0.01$ & 3.00 & 0.98 & $\operatorname{midz}$ & 5 \\
\hline 02:39:44.591 & $+07: 26: 59.62$ & $18.09 \pm 0.00$ & 2.92 & 0.73 & $\operatorname{midz}$ & 5 \\
\hline $02: 56: 27.355$ & $-18: 35: 49.70$ & $18.11 \pm 0.00$ & 2.88 & 0.99 & $\operatorname{midz}$ & 5 \\
\hline 03:05:33.395 & $+12: 57: 34.33$ & $18.07 \pm 0.01$ & 2.90 & 0.99 & $\operatorname{midz}$ & 5 \\
\hline $03: 18: 29.401$ & $+23: 34: 35.21$ & $18.32 \pm 0.01$ & 3.85 & 0.87 & highz & 3 \\
\hline 04:14:03.285 & $-10: 50: 03.75$ & $18.30 \pm 0.01$ & 2.83 & 0.98 & $\operatorname{midz}$ & 5 \\
\hline $04: 23: 28.876$ & $-27: 52: 23.82$ & $18.14 \pm 0.01$ & 2.92 & 0.96 & $\operatorname{midz}$ & 5 \\
\hline 04:44:07.833 & $+80: 34: 43.36$ & $18.24 \pm 0.01$ & 3.46 & 0.60 & highz & 4 \\
\hline $04: 46: 49.052$ & $-03: 54: 39.44$ & $18.48 \pm 0.01$ & 2.83 & 0.85 & $\operatorname{midz}$ & 5 \\
\hline $05: 05: 25.937$ & $+76: 49: 53.53$ & $17.77 \pm 0.01$ & 3.58 & 0.94 & highz & 1 \\
\hline $05: 34: 17.420$ & $+75: 44: 13.74$ & $17.27 \pm 0.00$ & 3.04 & 0.54 & $\operatorname{midz}$ & 2 \\
\hline 05:47:06.719 & $+79: 02: 21.45$ & $17.79 \pm 0.01$ & 3.20 & 0.89 & $\operatorname{midz}$ & 2 \\
\hline $05: 57: 01.236$ & $+68: 30: 27.86$ & $18.14 \pm 0.01$ & 2.87 & 1.00 & $\operatorname{midz}$ & 5 \\
\hline $06: 12: 25.946$ & $+66: 15: 22.70$ & $18.25 \pm 0.01$ & 3.15 & 0.90 & highz & 4 \\
\hline $06: 34: 29.752$ & $+56: 34: 42.36$ & $18.46 \pm 0.00$ & 3.02 & 0.91 & $\operatorname{midz}$ & 4 \\
\hline $06: 42: 53.018$ & $+59: 43: 45.50$ & $18.30 \pm 0.01$ & 3.84 & 0.96 & highz & 3 \\
\hline $06: 50: 56.448$ & $+72: 53: 14.65$ & $18.04 \pm 0.00$ & 4.49 & 0.83 & highz & 3 \\
\hline $06: 52: 41.984$ & $+54: 27: 40.60$ & $18.11 \pm 0.02$ & 3.65 & 0.99 & highz & 3 \\
\hline $06: 57: 27.418$ & $+57: 22: 11.94$ & $17.49 \pm 0.01$ & 3.61 & 0.92 & highz & 1 \\
\hline $07: 00: 32.592$ & $+56: 00: 27.17$ & $17.98 \pm 0.00$ & 3.04 & 0.76 & highz & 2 \\
\hline 07:08:02.482 & $+63: 15: 59.67$ & $17.20 \pm 0.00$ & 2.95 & 0.76 & $\operatorname{midz}$ & 3 \\
\hline $07: 14: 46.848$ & $+84: 25: 28.21$ & $17.81 \pm 0.01$ & 2.93 & 0.64 & $\operatorname{midz}$ & 3 \\
\hline $07: 15: 52.373$ & $+42: 10: 06.15$ & $17.62 \pm 0.01$ & 3.08 & 0.63 & $\operatorname{midz}$ & 2 \\
\hline $07: 17: 03.905$ & $+59: 02: 59.46$ & $18.40 \pm 0.01$ & 3.61 & 0.95 & highz & 3 \\
\hline $07: 32: 57.277$ & $+54: 52: 11.55$ & $17.80 \pm 0.01$ & 3.07 & 0.59 & $\operatorname{midz}$ & 2 \\
\hline 07:37:59.176 & $+54: 54: 44.01$ & $17.76 \pm 0.00$ & 2.88 & 0.96 & $\operatorname{midz}$ & 3 \\
\hline $07: 42: 23.031$ & $+68: 36: 31.53$ & $18.49 \pm 0.01$ & 3.95 & 0.96 & highz & 3 \\
\hline $07: 42: 58.216$ & $+61: 21: 10.97$ & $17.61 \pm 0.00$ & 3.62 & 0.94 & highz & 1 \\
\hline 07:51:07.041 & $+37: 11: 56.34$ & $18.19 \pm 0.01$ & 2.91 & 0.90 & $\operatorname{midz}$ & 5 \\
\hline $07: 51: 55.122$ & $+53: 53: 34.41$ & $18.48 \pm 0.00$ & 3.50 & 0.92 & highz & 4 \\
\hline $07: 52: 48.270$ & $+70: 24: 33.00$ & $18.16 \pm 0.01$ & 3.60 & 0.97 & highz & 3 \\
\hline $07: 55: 50.673$ & $+68: 47: 04.24$ & $17.68 \pm 0.01$ & 2.95 & 0.47 & $\operatorname{midz}$ & 3 \\
\hline 08:09:10.462 & $+59: 01: 25.22$ & $18.23 \pm 0.01$ & 3.00 & 0.94 & highz & 5 \\
\hline 08:23:56.195 & $+69: 08: 15.67$ & $18.27 \pm 0.00$ & 3.21 & 0.95 & highz & 4 \\
\hline 08:32:04.867 & $+57: 33: 15.31$ & $18.49 \pm 0.01$ & 3.29 & 0.94 & highz & 4 \\
\hline $13: 34: 19.002$ & $+26: 55: 34.63$ & $18.43 \pm 0.01$ & 3.17 & 0.88 & highz & 4 \\
\hline 23:05:05.917 & $+26: 47: 14.04$ & $18.27 \pm 0.01$ & 2.94 & 0.51 & $\operatorname{midz}$ & 5 \\
\hline
\end{tabular}

(This table is available in machine-readable form.) 


\section{Appendix E \\ SQL Query to Obtain the Pan-STARRS DR1 (PS1) Photometry}

We present the SQL query, which was used to obtain photometry from the Pan-STARRS DR1 catalog.

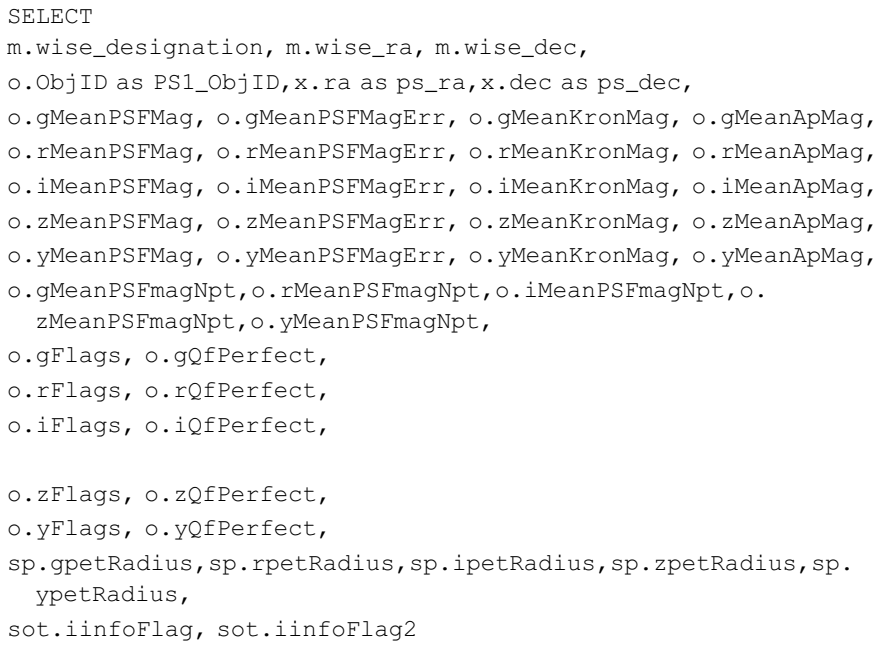

\section{ORCID iDs}

Jan-Torge Schindler (ib https://orcid.org/0000-00024544-8242

Xiaohui Fan (1D https://orcid.org/0000-0003-3310-0131 Yun-Hsin Huang (i) https://orcid.org/0000-0003-4955-5632 Minghao Yue (i) https://orcid.org/0000-0002-5367-8021 Jinyi Yang (iD https://orcid.org/0000-0001-5287-4242 Patrick B. Hall (i) https://orcid.org/0000-0002-1763-5825 Lukas Wenzl (i) https://orcid.org/0000-0001-5245-2058 Allison Hughes (iD https://orcid.org/0000-0002-1718-0402

Katrina C. Litke (i) https://orcid.org/0000-0002-4208-3532 Jon M. Rees (iD https://orcid.org/0000-0002-5376-3883

\section{References}

Abazajian, K. N., Adelman-McCarthy, J. K., Agüeros, M. A., et al. 2009, ApJS, 182, 543

Aihara, H., Arimoto, N., Armstrong, R., et al. 2018, PASJ, 70, S4

Akiyama, M., He, W., Ikeda, H., et al. 2018, PASJ, 70, S34

Astropy Collaboration, Price-Whelan, A. M., Sipőcz, B. M., et al. 2018, AJ, 156,123

Astropy Collaboration, Robitaille, T. P., Tollerud, E. J., et al. 2013, A\&A, 558,33

Bañados, E., Venemans, B. P., Decarli, R., et al. 2016, ApJS, 227, 11

Bañados, E., Venemans, B. P., Mazzucchelli, C., et al. 2018, Natur, 553, 473

Bañados, E., Venemans, B. P., Morganson, E., et al. 2014, AJ, 148, 14

Bishop, C. M. 2006, Pattern Recognition and Machine Learning (Berlin: Springer)

Boller, T., Freyberg, M. J., Trümper, J., et al. 2016, A\&A, 588, A103

Bovy, J., Hennawi, J. F., Hogg, D. W., et al. 2011, ApJ, 729, 141

Breiman, L. 2001, Machine Learn., 45, 5

Carliles, S., Budavári, T., Heinis, S., Priebe, C., \& Szalay, A. S. 2010, ApJ, 712,511

Carnall, A. C., Shanks, T., Chehade, B., et al. 2015, MNRAS, 451, L16

Carrasco, D., Barrientos, L. F., Pichara, K., et al. 2015, A\&A, 584, A44

Carrasco Kind, M., \& Brunner, R. J. 2013, MNRAS, 432, 1483

Chambers, K. C., Magnier, E. A., Metcalfe, N., et al. 2016, arXiv:1612.05560

Chehade, B., Carnall, A. C., Shanks, T., et al. 2018, MNRAS, 478, 1649

Clemens, J. C., Crain, J. A., \& Anderson, R. 2004, Proc. SPIE, 5492, 331 da Ângela, J., Shanks, T., Croom, S. M., et al. 2008, MNRAS, 383, 565

D'Isanto, A., \& Polsterer, K. L. 2018, A\&A, 609, A111

Dawson, K. S., Kneib, J.-P., Percival, W. J., et al. 2016, AJ, 151, 44

Dawson, K. S., Schlegel, D. J., Ahn, C. P., et al. 2013, AJ, 145, 10

Dubath, P., Rimoldini, L., Süveges, M., et al. 2011, MNRAS, 414, 2602

Eftekharzadeh, S., Myers, A. D., White, M., et al. 2015, MNRAS, 453, 2779

Eisenstein, D. J., Weinberg, D. H., Agol, E., et al. 2011, AJ, 142, 72

Fan, X., Hennawi, J. F., Richards, G. T., et al. 2004, AJ, 128, 515

Fan, X., Strauss, M. A., Richards, G. T., et al. 2006, AJ, 131, 1203

Fan, X., Strauss, M. A., Schneider, D. P., et al. 2001, AJ, 121, 54

Fan, X., Strauss, M. A., Schneider, D. P., et al. 2003, AJ, 125, 1649

Fan, X., White, R. L., Davis, M., et al. 2000, AJ, 120, 1167

Flesch, E. W. 2015, PASA, 32, e010

Górski, K. M., Hivon, E., Banday, A. J., et al. 2005, ApJ, 622, 759

Green, G. M., Schlafly, E. F., Finkbeiner, D., et al. 2018, MNRAS, 478, 651 Hunter, J. D. 2007, CSE, 9, 90

Jiang, L., Fan, X., Annis, J., et al. 2008, AJ, 135, 1057

Jiang, L., Fan, X., Bian, F., et al. 2009, AJ, 138, 305

Jiang, L., McGreer, I. D., Fan, X., et al. 2016, ApJ, 833, 222

Jones, E., Oliphant, T., Peterson, P., et al. 2001, SciPy: Open Source Scientific Tools for Python, https://www.scipy.org/

Kaiser, N., Aussel, H., Burke, B. E., et al. 2002, Proc. SPIE, 4836, 154

Kaiser, N., Burgett, W., Chambers, K., et al. 2010, Proc. SPIE, 7733, 77330E

Kashikawa, N., Ishizaki, Y., Willott, C. J., et al. 2015, ApJ, 798, 28

Magnier, E. 2006, in Proc. The Advanced Maui Optical and Space Surveillance Technologies Conf., ed. S. Ryan (Maui, HI: AMOS), E50

Magnier, E. 2007, in ASP Conf. Ser. 364, The Future of Photometric, Spectrophotometric and Polarimetric Standardization, ed. C. Sterken (San Francisco, CA: ASP), 153

Mainzer, A., Bauer, J., Grav, T., et al. 2011, ApJ, 731, 53

Martin, D. C., Fanson, J., Schiminovich, D., et al. 2005, ApJL, 619, L1 
Matsuoka, Y., Iwasawa, K., Onoue, M., et al. 2018a, ApJS, 237, 5

Matsuoka, Y., Onoue, M., Kashikawa, N., et al. 2016, ApJ, 828, 26

Matsuoka, Y., Onoue, M., Kashikawa, N., et al. 2018b, PASJ, 70, S35

Matsuoka, Y., Onoue, M., Kashikawa, N., et al. 2019, ApJL, 872, L2

Mazzucchelli, C., Bañados, E., Venemans, B. P., et al. 2017, ApJ, 849, 91

McGreer, I. D., Jiang, L., Fan, X., et al. 2013, ApJ, 768, 105

McKinney, W. 2011, in Proc. 9th Python Sci. Conf., ed. S. van der Walt \& J. Millman (Austin, TX: SciPy), 51

Morganson, E., De Rosa, G., Decarli, R., et al. 2012, AJ, 143, 142

Mortlock, D. J., Warren, S. J., Venemans, B. P., et al. 2011, Natur, 474, 616

Myers, A. D., Brunner, R. J., Nichol, R. C., et al. 2007, ApJ, 658, 85

Myers, A. D., Brunner, R. J., Richards, G. T., et al. 2006, ApJ, 638, 622

Myers, A. D., Palanque-Delabrouille, N., Prakash, A., et al. 2015, ApJS, 221, 27

Oke, J. B., \& Gunn, J. E. 1983, ApJ, 266, 713

Pâris, I., Petitjean, P., Aubourg, É., et al. 2012, A\&A, 548, A66

Pâris, I., Petitjean, P., Aubourg, É., et al. 2018, A\&A, 613, A51

Pedregosa, F., Varoquaux, G., Gramfort, A., et al. 2011, J. Machine Learn. Res., 12, 2825

Planck Collaboration, Ade, P. A. R., Aghanim, N., et al. 2016, A\&A, 594, A13

Pons, E., McMahon, R. G., Simcoe, R. A., et al. 2019, MNRAS, 484, 5142

Prochaska, J. X., Herbert-Fort, S., \& Wolfe, A. M. 2005, ApJ, 635, 123

Reed, S. L., Banerji, M., Becker, G. D., et al. 2019, MNRAS, 487, 1874

Reed, S. L., McMahon, R. G., Banerji, M., et al. 2015, MNRAS, 454, 3952

Reed, S. L., McMahon, R. G., Martini, P., et al. 2017, MNRAS, 468, 4702

Richards, G. T., Fan, X., Newberg, H. J., et al. 2002, AJ, 123, 2945

Richards, G. T., Kruczek, N. E., Gallagher, S. C., et al. 2011, AJ, 141, 167

Richards, G. T., Myers, A. D., Peters, C. M., et al. 2015, ApJS, 219, 39

Rodríguez-Torres, S. A., Comparat, J., Prada, F., et al. 2017, MNRAS, 468, 728

Ross, N. P., McGreer, I. D., White, M., et al. 2013, ApJ, 773, 14

Ross, N. P., Shen, Y., Strauss, M. A., et al. 2009, ApJ, 697, 1634

Salvato, M., Buchner, J., Budavári, T., et al. 2018, MNRAS, 473, 4937
Schindler, J.-T., Fan, X., McGreer, I. D., et al. 2017, ApJ, 851, 13

Schindler, J.-T., Fan, X., McGreer, I. D., et al. 2018, ApJ, 863, 144

Schindler, J.-T., Fan, X., McGreer, I. D., et al. 2019, ApJ, 871, 258

Schlegel, D. J., Finkbeiner, D. P., \& Davis, M. 1998, ApJ, 500, 525

Schmidt, T. M., Hennawi, J. F., Lee, K.-G., et al. 2018, arXiv:1810.05156

Schneider, D. P., Richards, G. T., Hall, P. B., et al. 2010, AJ, 139, 2360

Shen, Y., Strauss, M. A., Oguri, M., et al. 2007, AJ, 133, 2222

Simcoe, R. A., Sargent, W. L. W., \& Rauch, M. 2004, ApJ, 606, 92

Timlin, J. D., Ross, N. P., Richards, G. T., et al. 2018, ApJ, 859, 20

Tody, D. 1986, Proc. SPIE, 627, 733

Tody, D. 1993, in ASP Conf. Ser. 52, Astronomical Data Analysis Software and Systems II, ed. R. J. Hanisch, R. J. V. Brissenden, \& J. Barnes (San Francisco, CA: ASP), 173

Truemper, J. 1982, AdSpR, 2, 241

Vanden Berk, D. E., Richards, G. T., Bauer, A., et al. 2001, AJ, 122, 549

Venemans, B. P., Findlay, J. R., Sutherland, W. J., et al. 2013, ApJ, 779, 24

Venemans, B. P., McMahon, R. G., Warren, S. J., et al. 2007, MNRAS, 376, L76

Volonteri, M. 2012, Sci, 337, 544

Wang, F., Yang, J., Fan, X., et al. 2018a, arXiv:1810.11926

Wang, F., Yang, J., Fan, X., et al. 2018b, ApJL, 869, L9

White, M., Myers, A. D., Ross, N. P., et al. 2012, MNRAS, 424, 933

Willott, C. J., Delorme, P., Omont, A., et al. 2007, AJ, 134, 2435

Willott, C. J., Delorme, P., Reylé, C., et al. 2010, AJ, 139, 906

Worseck, G., \& Prochaska, J. X. 2011, ApJ, 728, 23

Worseck, G., Prochaska, J. X., Hennawi, J. F., \& McQuinn, M. 2016, ApJ, 825,144

Wu, X.-B., Hao, G., Jia, Z., Zhang, Y., \& Peng, N. 2012, AJ, 144, 49

Wu, X.-B., \& Jia, Z. 2010, MNRAS, 406, 1583

Yang, J., Wang, F., Fan, X., et al. 2019a, AJ, 157, 236

Yang, J., Wang, F., Fan, X., et al. 2019b, ApJ, 871, 199

York, D. G., Adelman, J., Anderson, J. E., Jr., et al. 2000, AJ, 120, 1579 